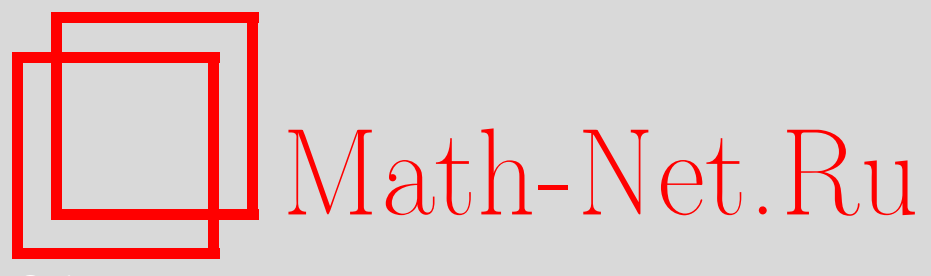

Вик. С. Куликов, Полугруппы разложений на множители и неприводимые компоненты пространства Гурвица, Изв. РАН. Сер. матем., 2011, том 75, выпуск 4, 49-90

DOI: https://doi.org/10.4213/im4500

Использование Общероссийского математического портала Math-Net.Ru подразумевает, что вы прочитали и согласны с пользовательским соглашением http://www . mathnet.ru/rus/agreement

Параметры загрузки:

IP : 3.85 .183 .62

26 апреля 2023 г., 15:29:22

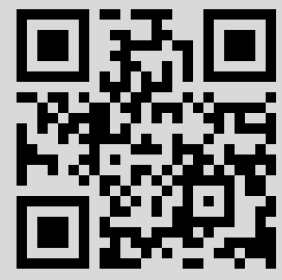


Вик. С. Куликов

\title{
Полугруппы разложений на множители и неприводимые компоненты пространства Гурвица
}

\begin{abstract}
Определена естественная структура полугруппы (изоморфной полугруппе разложений на множители единицы в симметрической группе) на множестве неприводимых компонент пространства Гурвица отмеченных накрытий степени $d$ проективной прямой $\mathbb{P}^{1}$ с фиксированными типами ветвлений. Доказано, что эта полугруппа является конечно определенной. Исследована также проблема, когда наборы типов ветвления однозначно определяют соответствующие неприводимые компоненты пространства Гурвица. В частности, полностью описано множество неприводимых компонент пространства Гурвица трехлистных накрытий проективной прямой.

Библиография: 14 наименований.
\end{abstract}

Ключевые слова: полугруппа, разложения на множители элемента группы, неприводимые компоненты пространства Гурвица.

\section{Введение}

Обычно, чтобы исследовать пространство Гурвица $\mathrm{HUR}_{d}\left(\mathbb{P}^{1}\right)$ накрытий проективной прямой $\mathbb{P}^{1}:=\mathbb{C P}^{1}$ степени $d$, используется следующий подход. Фиксируются группа Галуа $G$ накрытий, число $b$ точек ветвления и типы локальных монодромий ветвления (т. е. фиксируются наборы, состоящие из $b$ классов сопряженности элементов группы $G$ ), и после этого множество наборов, состоящих из представителей этих классов сопряженности, исследуется с точностью до так называемых преобразований Гурвица (см., например, [1]-[9]). Существует несколько других проблем (например, описать множество плоских алгебраических кривых с точностью до эквисингулярной деформации, или, более общо́, описать множество плоских псевдоголоморфных кривых с точностью до симплектической деформации, описать множество симплектических пучков Лефшеца с точностью до диффеоморфизмов), в которых также возникают аналогичные объекты, а именно конечные наборы, состоящие из элементов некоторых групп и рассматриваемые с точностью до преобразований Гурвица (см., например, [10]-[12]). (В случае плоских алгебраических и пседоголоморфных кривых, чтобы получить такие наборы, выбирается пучок прямых (псевдопрямых), задающий расслоение над проективной прямой $\mathbb{P}^{1}$.) Как было показано в [13], на множествах таких наборов, рассматриваемых с точностью до преобразований Гурвица, имеются естественные структуры полугрупп, а именно

Работа выполнена при финансовой поддержке РФФИ (грант № 11-01-00185), Программы Президента РФ "Поддержка ведущих научных школ" (грант НШ-4713.2010.1) и Лаборатории алгебраической геометрии ГУ-ВШЭ по гранту Правительства РФ (договор № 11.G34.31.0023). 
так называемые полугруппы разложений на множители в группах. Более того, если мы рассматриваем такие расслоения не только над всей проективной прямой $\mathbb{P}^{1}$, но и над дисками $D_{R}=\{z \in \mathbb{C}|| z \mid \leqslant R\}$, то эти структуры полугрупп имеют естественное геометрическое значение (см. [13]).

В 11 приведены основные определения и исследованы свойства полугрупп разложений на множители в конечных группах. В частности, доказано, что полугруппы разложений на множители единицы являются конечно определенными. Здесь мы также исследуем проблему: когда элемент полугруппы разложений на множители однозначно определяется своим типом и произведением?

В $\S 2$ более детально исследуются полугруппы разложений на множители в симметрических группах $\mathcal{S}_{d}$. Здесь мы доказываем теорему о стабилизации и полностью описываем полугруппу разложений на множители единицы в группе $\mathcal{S}_{3}$.

В $\S 3$ мы определяем естественную структуру полугруппы (а именно, полугруппы разложений на множители единицы в симметрической группе) на множестве неприводимых компонент пространства Гурвица отмеченных накрытий степени $d$ проективной прямой $\mathbb{P}^{1}$ с фиксированным множеством типов ветвлений и показываем, что эта структура индуцирует полугрупповую структуру на множестве неприводимых компонент пространства Гурвица $\mathrm{HUR}_{d}^{G}$ накрытий Галуа проективной прямой $\mathbb{P}^{1}$ с группой Галуа $G$, не имеющей внешних автоморфизмов. Кроме того, результаты, полученные в $§ 1,2$, применяются к решению вопроса о том, когда неприводимые компоненты пространства $\operatorname{HUR}_{d}\left(\mathbb{P}^{1}\right)$ однозначно определяются наборами типов локальных монодромий накрытий.

\section{§1. Полугруппы над группами}

1.1. Полугруппы разложений на множители. Набор $(S, G, \alpha, \lambda)$, состоящий из полугруппы $S$, группы $G$ и гомоморфизмов $\alpha: S \rightarrow G, \lambda: G \rightarrow$ $\operatorname{Aut}(S)$, называется полугруппой $S$ над группой $G$, если для всех $s_{1}, s_{2} \in S$ выполнены равенства

$$
s_{1} \cdot s_{2}=\rho\left(\alpha\left(s_{1}\right)\right)\left(s_{2}\right) \cdot s_{1}=s_{2} \cdot \lambda\left(\alpha\left(s_{2}\right)\right)\left(s_{1}\right),
$$

где $\rho(g)=\lambda\left(g^{-1}\right)$.

Пусть $\left(S_{1}, G_{1}, \alpha_{1}, \lambda_{1}\right)$ и $\left(S_{2}, G_{2}, \alpha_{2}, \lambda_{2}\right)$ - полугруппы соответственно над группами $G_{1}$ и $G_{2}$. Пара $\left(h_{1}, h_{2}\right)$ гомоморфизмов $h_{1}: S_{1} \rightarrow S_{2}$ и $h_{2}: G_{1} \rightarrow G_{2}$ называется гомоморфизмом полугрупп над группами, если:

(i) $h_{2} \circ \alpha_{1}=\alpha_{2} \circ h_{1}$;

(ii) $\lambda_{2}\left(h_{2}(g)\right)\left(h_{1}(s)\right)=h_{1}\left(\lambda_{1}(g)\right)(s)$ для всех $s \in S_{1}$ и всех $g \in G_{1}$.

Определенные ниже полугруппы разложений на множители являются основными (для наших целей) примерами полугрупп над группами.

Пусть $O \subset G$ - инвариантное относительно действия внутренними автоморфизмами подмножество группы $G$. Назовем пару $(G, O)$ оснащенной группой. Сопоставим множеству $O$ алфавит $X=X_{O}=\left\{x_{g} \mid g \in O\right\}$ и для каждой пары букв $x_{g_{1}}, x_{g_{2}} \in X, g_{1} \neq g_{2}$, обозначим через $R_{g_{1}, g_{2} ; l}$ и $R_{g_{1}, g_{2} ; r}$ следующие соотношения: $R_{g_{1}, g_{2} ; l}$ - это соотношение вида

$$
x_{g_{1}} \cdot x_{g_{2}}=x_{g_{2}} \cdot x_{g_{2}^{-1} g_{1} g_{2}},
$$


если $g_{2} \neq \mathbf{1}$, и вида $x_{g_{1}} \cdot x_{\mathbf{1}}=x_{g_{1}}$, если $g_{2}=\mathbf{1}$, а $R_{g_{1}, g_{2} ; r}$ - это соотношение вида

$$
x_{g_{1}} \cdot x_{g_{2}}=x_{g_{1} g_{2} g_{1}^{-1}} \cdot x_{g_{1}},
$$

если $g_{1} \neq \mathbf{1}$, и вида $x_{\mathbf{1}} \cdot x_{g_{2}}=x_{g_{2}}$, если $g_{1}=\mathbf{1}$.

Положим

$$
\mathcal{R}=\left\{R_{g_{1}, g_{2} ; r}, R_{g_{1}, g_{2} ; l} \mid\left(g_{1}, g_{2}\right) \in O \times O, g_{1} \neq g_{2}\right\} .
$$

С помощью множества соотношений $\mathcal{R}$ определим полугруппу

$$
S(G, O)=\left\langle x_{g} \in X \mid R \in \mathcal{R}\right\rangle
$$

и назовем ее полугруппой разложений на множители элементов из $G$ с множителями из $O$.

Определим также гомоморфизм $\alpha: S(G, O) \rightarrow G$, заданный на порождающих элементах $x_{g} \in X$ формулой $\alpha\left(x_{g}\right)=g$, и назовем его гомоморфизмом произведения.

Кроме того, определим действие $\lambda$ группы $G$ на множестве $X$ следующим образом:

$$
x_{a} \in X \mapsto \lambda(g)\left(x_{a}\right)=x_{g^{-1} a g} \in X .
$$

Легко видеть, что множество соотношений $\mathcal{R}$ инвариантно относительно действия $\lambda$. Поэтому действие $\lambda$ определяет гомоморфизм $\lambda: G \rightarrow \operatorname{Aut}(S(G, O))$ (действие сопряжениями). Действие $\lambda(g)$ на $S(G, O)$ называется одновременнъц сопряжением на элемент $g$. Положим $\lambda_{S}=\lambda \circ \alpha$ и $\rho_{S}=\rho \circ \alpha$.

УтвЕРЖДЕНИЕ 1.1 [11]. Для всех $s_{1}, s_{2} \in S(G, O)$ имеем

$$
s_{1} \cdot s_{2}=s_{2} \cdot \lambda_{S}\left(s_{2}\right)\left(s_{1}\right)=\rho_{S}\left(s_{1}\right)\left(s_{2}\right) \cdot s_{1} .
$$

Из утверждения 1.1 следует, что $(S(G, O), G, \alpha, \lambda)$ является полугруппой над группой $G$. Если группа $G$ фиксирована, то полугруппа $S(G, O)$ будет обозначаться $S_{O}$. Элемент полугруппы $S_{O}$, заданный словом $x_{g_{1}} \ldots x_{g_{n}}$, будет обозначаться через $x_{g_{1}} \cdot \ldots \cdot x_{g_{n}}$.

Отметим, что сопоставление $S:(G, O) \mapsto(S(G, O), G, \alpha, \lambda)$ является функтором из категории оснащенных групп в категорию полугрупп над группами. В частности, если $O_{1} \subset O_{2}$ - два подмножества группы $G$, инвариантные относительно внутренних автоморфизмов группы $G$, то тождественное отображение $\mathrm{id}: G \rightarrow G$ определяет вложение $\operatorname{id}_{O_{1}, O_{2}}: S\left(G, O_{1}\right) \rightarrow S\left(G, O_{2}\right)$. Таким образом, для каждой группы $G$ полугруппа $S_{G}=S(G, G)$ является универсальной полугруппой разложений на множители в группе $G$, т. е. каждая полугруппа $S_{O}$ над группой $G$ канонически вкладывается в $S_{G}$ с помощью отображения $\operatorname{id}_{O, G}$.

Пусть $\Gamma$ - подгруппа группы $G$. Обозначим $S_{O, \Gamma}=\left\{s \in S_{O} \mid \alpha(s) \in \Gamma\right\}$. Очевидно, что $S_{O, \Gamma}$ является подполугруппой полугруппы $S_{O}$ и если Г является нормальным делителем группы $G$, то $S_{O, \Gamma}$ является полугруппой над группой $G$. В частности, подполугруппа $S_{O, \mathbf{1}}$ (здесь $\Gamma=\{\mathbf{1}\}$ ) является важным примером таких подполугрупп.

Группа $G$ действует на себе самой внутренними автоморфизмами, т. е. для любой группы $G$ определен естественный гомоморфизм $h: G \rightarrow \operatorname{Aut}(G)$ (действие образа $h(g)=a$ элемента $g$ на $G$ задается формулой $\left(g_{1}\right) a=g^{-1} g_{1} g$ для 
всех $\left.g_{1} \in G\right)$. Легко видеть, что гомоморфизм $h$ определяет на $S_{G}$ структуру полугруппы над группой $A=\operatorname{Aut}(G)$, где гомоморфизм $\alpha_{A}: S_{G} \rightarrow \operatorname{Aut}(G)$ - это композиция $h \circ \alpha$, а элемент $a \in \operatorname{Aut}(G)$ действует на $S_{G}$ по правилу $x_{g} \mapsto x_{(g) a}$. Легко видеть, что подполугруппа $S_{G, \mathbf{1}}$ является инвариантной относительно действия группы $\operatorname{Aut}(G)$ на $S_{G}$. Следовательно, полугруппа $S_{G, 1}$ также может быть рассмотрена как полугруппа над группой $\operatorname{Aut}(G)$.

Сопоставим каждому элементу $s=x_{g_{1}} \cdot \ldots \cdot x_{g_{n}} \in S_{O}, g_{i} \neq \mathbf{1}$, число $\ln (s)=$ $n-$ длину элемента $s$. Легко видеть, что $\ln : S_{O} \rightarrow \mathbb{Z}_{\geqslant 0}=\{\mathbf{a} \in \mathbb{Z} \mid \mathbf{a} \geqslant 0\}$ является гомоморфизмом полугрупп.

Для каждого элемента $s=x_{g_{1}} \cdot \ldots \cdot x_{g_{n}} \in S_{O}$ обозначим через $G_{s}$ подгруппу группы $G$, порожденную образами $\alpha\left(x_{g_{1}}\right)=g_{1}, \ldots, \alpha\left(x_{g_{n}}\right)=g_{n}$ множителей $x_{g_{1}}, \ldots, x_{g_{n}}$.

УтВеРЖДЕНИЕ 1.2. Полугруппа $G_{s}$ группы $G$ корректно определена, m.е. ее определение не зависит от представления элемента s в виде произведения порождающих элементов $x_{g_{i}} \in X_{O}$.

Доказательства утверждения 1.2 и следующего предложения являются очень простыми и потому не приводятся.

ПРЕДЛОЖЕНИЕ 1.1 [11]. Пусть $(G, O)$ - оснащенная группа, и пусть $s \in S_{O}$. Имеем:

1) $\operatorname{ker} \lambda$ совпадает с иентрализатором $C_{O}$ группь $G_{O}$ в группе $G$;

2) если $\alpha(s)$ принадлежит центру $Z\left(G_{s}\right)$ группы $G_{s}$, то для каждого $g \in G_{s}$ действие $\lambda(g)$ оставляет неподвижным элемент $s \in S_{O}$;

3) если $\alpha\left(s \cdot x_{g}\right)$ принадлежит центру $Z\left(G_{s \cdot x_{g}}\right)$ группь $G_{s \cdot x_{g}}$, mо $s \cdot x_{g}=x_{g} \cdot s$;

4) если $\alpha(s)=\mathbf{1}$, то $s \cdot s^{\prime}=s^{\prime} \cdot s$ для любого $s^{\prime} \in S_{G}$.

УтВЕРЖДЕНИЕ 1.3. Для каждой оснащенной группъ $(G, O)$ nолугруппа $S_{O, 1}$ содержится в центре полугруппь $S_{G} u$, в частности, является коммутативной полугруппой.

ДокАзАТЕЛЬство следует из предложения 1.1,4).

Легко видеть, что если порядок элемента $g \in O$ равен $n$, то $x_{g}^{n} \in S_{O, \mathbf{1}}$.

Лемма 1.1. Пусть $s \in S_{O, \mathbf{1}}, u$ пусть $s_{1} \in S_{O}-$ такой элемент, что $G_{s_{1}}=G_{O}$. Тогда имеет место равенство

$$
s \cdot s_{1}=\lambda(g)(s) \cdot s_{1}
$$

для всех $g \in G_{O}$. В частности, если $C \subset O$-некоторый класс сопряженности элементов порядка $n_{C}$, а элемент $s \in S_{O}$ таков, что $G_{s}=G$, то для любих $g_{1}, g_{2} \in C$ имеем

$$
x_{g_{1}}^{n_{C}} \cdot s=x_{g_{2}}^{n_{C}} \cdot s .
$$

ДоказАтельство. Равенство (1.4) доказано в [5]. Доказательство равенства (1.3) аналогично.

Для каждой подгруппы $H$ группы $G$ положим

$$
S_{O}^{H}=S(G, O)^{H}=\left\{s \in S(G, O) \mid G_{s}=H\right\}
$$


и $S_{O, \mathbf{1}}^{H}=S_{O, \mathbf{1}} \cap S_{O}^{H}$. Тогда легко видеть, что полугруппа $S_{O}^{H}$ (соответственно, $\left.S_{O, 1}^{H}\right)$ изоморфна полугруппе $S(H, H \cap O)^{H}$ (соответственно, полугруппе $\left.S(H, H \cap O)_{1}^{H}\right)$, причем изоморфизм индуцирован вложением $H \hookrightarrow G$.

1.2. $C$-группы, ассоциированные с оснащенными группами, и гомоморфизм типа. Пусть $(G, O)$ - оснащенная группа такая, что $\mathbf{1} \notin O$, и пусть множество $O$ состоит из объединения $m$ классов сопряженности: $O=$ $C_{1} \cup \cdots \cup C_{m}$

Группа $\widehat{G}_{O}$, порожденная алфавитом $Y_{O}=\left\{y_{g} \mid g \in O\right\}$ (так называемые $C$-порождающие), элементы которого связаны соотношениями

$$
y_{g_{1}} y_{g_{2}}=y_{g_{2}} y_{g_{2}^{-1} g_{1} g_{2}}=y_{g_{1} g_{2} g_{1}^{-1}} y_{g_{1}}, \quad y_{g_{1}}, y_{g_{2}} \in Y_{O}
$$

называется $C$-группой, ассочиированной с оснащенной группой $(G, O)$. Очевидно, что отображения $x_{g} \mapsto y_{g}$ и $y_{g} \mapsto g$ определяют два гомоморфизма $\beta: S(G, O) \rightarrow \widehat{G}_{O}$ и $\gamma: \widehat{G}_{O} \rightarrow G$ таких, что $\alpha=\gamma \circ \beta$. Элементы, принадлежащие $\operatorname{Im} \beta$, называются положительными элементами группы $\widehat{G}_{O}$.

$C$-группа $\widehat{G}_{O}$, ассоциированная с оснащенной группой $(G, O)$, имеет аналогичные свойства, что и полугруппа $S_{O}$. Например, как и в случае полугрупп разложений на множители, легко проверить, что для произвольных $\hat{g} \in \widehat{G}_{O}$ и $g_{1} \in O$ соотношение

$$
\hat{g}^{-1} y_{g_{1}} \hat{g}=y_{g^{-1} g_{1} g}
$$

является следствием соотношений (1.5), где $g=\gamma(\hat{g})$.

Обозначим через $\widehat{O}$ подмножество $\left\{y_{g} \mid g \in O\right\}$ группы $\widehat{G}_{O}$. Из соотношений $(1.5),(1.6)$ следует, что $\widehat{O}$ является инвариантным множеством относительно действия группы $\widehat{G}_{O}$ на себе внутренними автоморфизмами.

УТВЕРЖДЕНИЕ 1.4. Пусть $(G, O)$ - оснащенная группа. Тогда полугруппь $S(G, O)$ и $S\left(\widehat{G}_{O}, \widehat{O}\right)$ естественньм образом изоморфны друг другу.

ДокАзАТЕЛЬство. Легко видеть, что согласно соотношениям (1.5), (1.6) отображение $\xi: S\left(\widehat{G}_{O}, \widehat{O}\right) \rightarrow S(G, O)$, заданное формулой $\xi\left(x_{y_{g}}\right)=x_{g}$ для $g \in O$, индуцирует изоморфизм полугрупп.

Следующее предложение является непосредственным следствием соотношений (1.5), (1.6) (см., например, [14]).

ПРЕДЛОЖЕНИЕ 1.2. Для любой оснащенной полугруппъ $(G, O)$ имеет место равенство

$$
Z\left(\widehat{G}_{O}\right)=\gamma^{-1}\left(Z\left(G_{O}\right)\right),
$$

где $Z\left(G_{O}\right)$ и $Z\left(\widehat{G}_{O}\right)$ - центры соответственно групп $G_{O}$ и $\widehat{G}_{O}$.

Легко видеть, что первая группа гомологий $H_{1}\left(\widehat{G}_{O}, \mathbb{Z}\right)=\widehat{G}_{O} /\left[\widehat{G}_{O}, \widehat{G}_{O}\right]$ группы $\widehat{G}_{O}$ является свободной абелевой группой ранга $m$. Пусть ab: $\widehat{G}_{O} \rightarrow$ $H_{1}\left(\widehat{G}_{O}, \mathbb{Z}\right)$ - естественный эпиморфизм. Группа $H_{1}\left(\widehat{G}_{O}, \mathbb{Z}\right) \simeq \mathbb{Z}^{m}$ порождается элементами $\mathrm{ab}\left(y_{g_{i}}\right)=(0, \ldots, 0,1,0, \ldots, 0)$, где $g_{i} \in C_{i}$ (1 стоит на $i$-м месте).

Гомоморфизм полугрупп $\tau=\mathrm{ab} \beta: S(G, O) \rightarrow \mathbb{Z}_{\geqslant 0}^{m} \subset \mathbb{Z}^{m}$ называется гомоморфизмом типа, а образ $\tau(s)$ элемента $s \in S(G, O)$ называется типом 
элемента $s$. Если $O$ состоит только из одного класса сопряженности, то гомоморфизм $\tau$ совпадает с гомоморфизмом $\ln : S(G, O) \rightarrow \mathbb{Z}_{\geqslant 0}$ (поэтому в данном случае мы будем отождествлять эти гомоморфизмы).

Лемма 1.2. Любой элемент $\hat{g} C$-группъ $\widehat{G}_{O}$, ассоциированной с оснащенной группой $(G, O)$, может быть представлен в виде

$$
\hat{g}=\hat{g}_{1} \hat{g}_{2}^{-1}
$$

где $\hat{g}_{1}, \hat{g}_{2}$ - некоторые положительные элементы. В частности, $\hat{g} \in \widehat{G}_{O}^{\prime}=$ $\left[\widehat{G}_{O}, \widehat{G}_{O}\right]$ тогда и только тогда, когда $\mathrm{ab}\left(\hat{g}_{1}\right)=\operatorname{ab}\left(\hat{g}_{2}\right)$ для элементов $\hat{g}_{1} u$ $\hat{g}_{2}$, участвующих в представлении (1.7) элемента $\hat{g}$ в виде отношения двух положительных элементов.

ДокАЗАтЕльство. Представим элемент $\hat{g}$ в виде $\hat{g}=y_{g_{i_{1}}}^{\varepsilon_{1}} \ldots y_{g_{i_{k}}}^{\varepsilon_{k}}$, где $g_{i_{j}} \in O$ и $\varepsilon_{j}= \pm 1$. Чтобы доказать лемму, достаточно заметить, что согласно соотношениям (1.5) имеет место равенство $y_{g_{2}}^{-1} y_{g_{1}}=y_{g_{2}^{-1} g_{1} g_{2}} y_{g_{2}}^{-1}$ для любых $g_{1}, g_{2} \in O$.

УтвеРЖДЕНИЕ 1.5. Пусть $(G, O)$ - оснащенная конечная группа. Гомоморфизм $\beta: S_{O} \rightarrow \widehat{G}_{O}$ является вложением тогда и только тогда, когда $O \subset Z\left(G_{O}\right)$, т.е. когда $G_{O}$ является абелевой группой.

ДокАЗАТЕЛЬСтво. Пусть $O=C_{1} \cup \cdots \cup C_{m}$ - разложение в объединение классов сопряженности. Легко видеть, что если $O \subset Z\left(G_{O}\right)$, то $\widehat{G}_{O} \simeq \mathbb{Z}^{|O|}$, где изоморфизм индуцирован гомоморфизмом $\mathrm{ab}$, и в этом случае полугруппа $S_{O}$ может быть отождествлена с полугруппой $\mathbb{Z}_{\geqslant 0}^{|O|} \subset \mathbb{Z}^{|O|}$.

Если $O \not \subset Z\left(G_{O}\right)$, то существует класс сопряженности $C_{i} \subset O$, состоящий по крайней мере из двух элементов, скажем $g_{1}$ и $g_{2}$. Пусть $n-$ их порядок в группе $G$. Тогда легко видеть, что $x_{g_{1}}^{n} \neq x_{g_{2}}^{n}$ в полугруппе $S_{O}$. С другой стороны, их образы $y_{g_{1}}^{n}=\beta\left(x_{g_{1}}^{n}\right)$ и $y_{g_{2}}^{n}=\beta\left(x_{g_{2}}^{n}\right)$ совпадают в $\widehat{G}_{O}$. Действительно, не ограничивая общности, мы можем считать, что найдется такой элемент $g \in G_{O}$, что $g_{2}=g^{-1} g_{1} g$. Рассмотрим элемент $\hat{g} \in \gamma^{-1}(g)$. Имеем

$$
\hat{g}^{-1} y_{g_{1}}^{n} \hat{g}=\left(\hat{g}^{-1} y_{g_{1}} \hat{g}\right)^{n}=y_{g^{-1} g_{1} g}^{n}=y_{g_{2}}^{n},
$$

но согласно предложению 1.2 элементы $y_{g_{1}}^{n}$ и $y_{g_{2}}^{n}$ принадлежат центру $Z\left(\widehat{G}_{O}\right)$. Следовательно, $y_{g_{1}}^{n}=y_{g_{2}}^{n}$.

1.3. Эквивалентность Гурвица. Как и выше, пусть $O$ - подмножество группы $G$, инвариантное относительно внутренних автоморфизмов группы $G$. Рассмотрим множество

$$
O^{n}=\left\{\left(g_{1}, \ldots, g_{n}\right) \mid g_{i} \in O\right\}
$$

всех упорядоченных $n$-наборов элементов из $O$, и пусть $\mathrm{Br}_{n}$ - группа кос с $n$ нитями. Зафиксируем множество $\left\{a_{1}, \ldots, a_{n-1}\right\}$ так называемых стандартных (или артиновых) порождающих группы $\mathrm{Br}_{n}$, т. е. порождающих группу $\mathrm{Br}_{n}$ элементов, связанных соотношениями

$$
\begin{gathered}
a_{i} a_{i+1} a_{i}=a_{i+1} a_{i} a_{i+1}, \quad 1 \leqslant i \leqslant n-1, \\
a_{i} a_{k}=a_{k} a_{i}, \quad|i-k| \geqslant 2 .
\end{gathered}
$$


Группа $\mathrm{Br}_{n}$ действует на $O^{n}$ следующим образом:

$$
\left(\left(g_{1}, \ldots, g_{i-1}, g_{i}, g_{i+1}, g_{i+2}, \ldots, g_{n}\right)\right) a_{i}=\left(g_{1}, \ldots, g_{i-1}, g_{i} g_{i+1} g_{i}^{-1}, g_{i}, g_{i+2}, \ldots, g_{n}\right) \text {. }
$$

Обычно эти действия стандартных порождающих $a_{i} \in \mathrm{Br}_{n}$ и их обратных на множестве $O^{n}$ называются преобразованиями Гурвища. Два элемента из $O^{n}$ называются гурвиц-эквивалентными, если один из них может быть преобразован в другой с помощью конечной последовательности преобразований Гурвица, т. е. если они принадлежат одной и той же орбите действия группы $\mathrm{Br}_{n}$.

На множестве $O^{n}$ определено естественное отображение $\alpha: O^{n} \rightarrow G$ (отображение произведения), заданное формулой

$$
\alpha\left(\left(g_{1}, \ldots, g_{n}\right)\right)=g_{1} \ldots g_{n},
$$

и элемент $\left(g_{1}, \ldots, g_{n}\right) \in O^{n}$ называется разложением на множители элемента $g=\alpha\left(\left(g_{1}, \ldots, g_{n}\right)\right) \in G$ с множителями из $O$.

Также имеется естественное отображение $\varphi: O^{n} \rightarrow S(G, O)$, переводящее $\left(g_{1}, \ldots, g_{n}\right)$ в $s=x_{g_{1}} \cdot \ldots \cdot x_{g_{n}}$.

УтВеРЖДЕНИЕ 1.6. Два разложения на множители $y, z \in O^{n}$ гурвиц-эквивалентны тогда и только тогда, когда $\varphi(y)=\varphi(z)$.

ДокАЗАТЕЛЬСТво очевидно.

ЗАмечАНИЕ 1.1. Ниже в соответствии с утверждением 1.6 мы будем отождествлять классы гурвиц-эквивалентных разложений на множители из $O$ с их образами в полугруппе $S(G, O)$.

Определим также действие группы $G$ сопряжениями на множестве $O^{n}$ :

$$
\lambda(g)\left(\left(g_{1}, \ldots, g_{n}\right)\right)=\left(g^{-1} g_{1} g, \ldots, g^{-1} g_{n} g\right) .
$$

Очевидно, что это действие совпадает при отображении $\varphi$ с определенным выше действием $\lambda$ группы $G$ на $S(G, O)$.

Обозначим через $W=W(O)$ множество всех слов алфавита $X=X_{O \backslash\{\mathbf{1}\}}$ и через $W_{n}$ его подмножество, состоящее из слов длины $n$. В дальнейшем мы будем отождествлять элементы множества $O^{n}$ с элементами из $W_{n}$ (отождествление: $\left.\left(g_{1}, \ldots, g_{n}\right) \in O^{n} \leftrightarrow x_{g_{1}} \ldots x_{g_{n}} \in W_{n}\right)$. Положим

$$
W(s)=\{w \in W \mid \varphi(w)=s \in S(G, O)\} .
$$

1.4. Неперфорированные подполугруппы полугруппы $\mathbb{Z}_{\geqslant 0}^{m}$. Ниже нам понадобятся некоторые факты о подполугруппах в полугруппе $\mathbb{Z}_{\geqslant 0}^{m}$.

Подполугруппа $S$ полугруппы $\mathbb{Z}_{\geqslant 0}^{m}$ называется неперфорированной, если для каждого элемента $\mathbf{a} \in S$ и любого $\mathbf{b} \in \mathbb{Z}_{\geqslant 0}^{m}$ имеем $\mathbf{a}+\mathbf{b} \in S$. Заметим, что если $S_{1}$ и $S_{2}$ - неперфорированные подполугруппы, то $S_{1} \cup S_{2}$ и $S_{1} \cap S_{2}$ также являются неперфорированными подполугруппами. Элемент а неперфорированной подполугруппы $S$ называется верииной подполугруппы $S$, если не существует элементов $\mathbf{b} \in S$ и $\mathbf{c} \in \mathbb{Z}_{\geqslant 0}^{m} \backslash\{\mathbf{0}\}$ таких, что $\mathbf{a}=\mathbf{b}+\mathbf{c}$. Обозначим через $O(S)$ множество всех вершин неперфорированной подполугруппы $S$. Неперфорированная подполугруппа $S$ с единственной вершиной называется простой. Легко видеть, что если а - вершина простой неперфорированной подполугруппы $S$, то

$$
S=F_{\mathbf{a}}=\left\{\mathbf{c}=\mathbf{a}+\mathbf{b} \in \mathbb{Z}_{\geqslant 0}^{m} \mid \mathbf{b} \in \mathbb{Z}_{\geqslant 0}^{m}\right\} .
$$


Очевидно, что любая неперфорированная подполугруппа $S$ может быть представлена в виде объединения простых неперфорированных подполугрупп, например

$$
S=\bigcup_{\mathbf{a} \in S} F_{\mathbf{a}}
$$

Пусть $A$ - некоторое подмножество в $S$, и пусть $S$ представлена в виде объединения простых неперфорированных подполугрупп:

$$
S=\bigcup_{\mathbf{a} \in A} F_{\mathbf{a}}
$$

Скажем, что представление (1.9) является минимальным, если

$$
S \neq \bigcup_{\mathbf{a} \in A \backslash\left\{\mathbf{a}_{0}\right\}} F_{\mathbf{a}}
$$

для всех $\mathbf{a}_{0} \in A$.

УтвеРЖДЕНИЕ 1.7. Для неперфорированной подполугруппы $S \subset \mathbb{Z}_{\geqslant 0}^{m}$ существует единственное минимальное представление в виде объединения простых неперфорированных подполугрупп, а именно

$$
S=\bigcup_{\mathbf{a} \in O(S)} F_{\mathbf{a}}
$$

ДокАЗАТЕЛЬство. Из определения вершин следует, что если $S=\bigcup F_{\mathbf{a}_{i}}$ представление в виде объединения простых неперфорированных подполугрупп и $\mathbf{a}-$ вершина подполугруппы $S$, то $\mathbf{a}=\mathbf{a}_{i}$ для некоторого $i$.

Предположим, что множество

$$
C=S \backslash \bigcup_{\mathbf{a} \in O(S)} F_{\mathbf{a}}
$$

не пусто, тогда существует элемент $\mathbf{c}_{0}=\left(c_{1,0}, \ldots, c_{m, 0}\right) \in C$ такой, что $c_{m, 0}=$ $\min c_{m}$ для $\left(c_{1}, \ldots, c_{m}\right) \in C, c_{m-1,0}=\min c_{m-1}$ для $\left(c_{1}, \ldots, c_{m-1}, c_{m, 0}\right) \in C, \ldots$

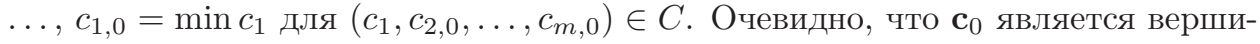
ной подполугруппы $S$.

ПрЕДЛОЖЕНИЕ 1.3. Каждая возрастающая последовательность неперборированных подполугрупп полугруппы $\mathbb{Z}_{\geqslant 0}^{m}$

$$
S_{1} \subset S_{2} \subset S_{3} \subset \cdots
$$

такая, что $S_{i} \neq S_{i+1}$, является конечной.

ДокАзАтельство. В случае $m=1$ предложение является очевидным. Применим индукцию по $m$. Рассмотрим возрастающую последовательность неперфорированных подполугрупп $S_{1} \subset S_{2} \subset S_{3} \subset \cdots \subset \mathbb{Z}_{\geqslant 0}^{m}, m \geqslant 2$. Обозначим $P_{j}=\left\{\left(z_{1}, \ldots, z_{m}\right) \in \mathbb{Z}_{\geqslant 0}^{m} \mid z_{m}=j\right\}$, и пусть $S_{i, j}=S_{i} \cap P_{j}$. Тогда $S_{i, j}$ также могут быть рассмотрены как неперфорированные подполугруппы полугруппы $\mathbb{Z}_{\geqslant 0}^{m-1}$ (если мы "забудем" о последней координате). По предположению индукции 
возрастающие последовательности $S_{1, j} \subset S_{2, j} \subset S_{3, j} \subset \cdots$ должны стабилизироваться при каждом $j$. Обозначим через $\bar{S}_{j}=S_{i(j), j}$ первые наибольшие полугруппы в этих последовательностях.

Рассмотрим отображение sh: $\mathbb{Z}_{\geqslant 0}^{m} \rightarrow \mathbb{Z}_{\geqslant 0}^{m}$, заданное формулой

$$
\operatorname{sh}\left(\left(z_{1}, \ldots, z_{m-1}, z_{m}\right)\right)=\left(z_{1}, \ldots, z_{m-1}, z_{m}+1\right) .
$$

Из определения неперфорированных подполугрупп следует, что sh: $S_{i, j} \rightarrow$ $S_{i, j+1}$ является вложением. Следовательно, мы можем (и будем) отождествлять полугруппы $S_{i, j}$ с подполугруппами $\operatorname{sh}^{n}\left(S_{i, j}\right)$ полугрупп $S_{i, j+n}$. Кроме того, из определения неперфорированных подполугрупп также следует, что если $j_{1}<j_{2}$, то $\bar{S}_{j_{1}}=S_{i\left(j_{1}\right), j_{1}} \subset \bar{S}_{j_{2}}=S_{i\left(j_{2}\right), j_{2}}$. В результате мы получаем возрастающую последовательность неперфорированных подполугрупп

$$
S_{i(0), 0} \subset S_{i(1), 1} \subset S_{i(2), 2} \subset \cdots \subset \mathbb{Z}_{\geqslant 0}^{m-1}
$$

Она должна стабилизироваться. Легко видеть, что если $S_{i\left(j_{0}\right), j_{0}}$ - наибольшая полугруппа, то $S_{i\left(j_{0}\right)}=S_{i\left(j_{0}\right)+1}=S_{i\left(j_{0}\right)+2}=\cdots$.

СлеДСТвИЕ 1.1. Множество вершин $O(S)$ неперфорированной подполугруппы $S \subset \mathbb{Z}_{\geqslant 0}^{m}$ непусто и конечно.

ДокАЗАТЕЛЬСтво. Если множество $O(S)=\left\{\mathbf{a}_{1}, \mathbf{a}_{2}, \mathbf{a}_{3}, \ldots\right\}$ является бесконечным, то согласно утверждению 1.7 мы будем иметь бесконечную возрастающую последовательность

$$
F_{\mathbf{a}_{1}} \subset F_{\mathbf{a}_{1}} \cup F_{\mathbf{a}_{2}} \subset F_{\mathbf{a}_{1}} \cup F_{\mathbf{a}_{2}} \cup F_{\mathbf{a}_{3}} \subset \cdots,
$$

что противоречит предложению 1.3.

\section{5. Конечная определенность некоторых подполугрупп полугруп-} пы $S(G, O)$. Пусть $(G, O)$ - конечная оснащенная группа. Согласно определению полугруппа $S_{O}$ является конечно определенной. С геометрической точки зрения наиболее интересными подполугруппами полугруппы $S_{G}$ являются полугруппы $S_{O, \mathbf{1}}$ и $S_{O, \mathbf{1}}^{G}=\left\{s \in S_{O, \mathbf{1}} \mid G_{s}=G\right\}$. (Отметим, что полугруппа $S_{O, \mathbf{1}}^{G}$ не пуста тогда и только тогда, когда $G_{O}=G$.) В этом пункте мы покажем, что полугруппы $S_{O, 1}$ являются конечно определенными, но для полугрупп $S_{O, 1}^{G}$ свойство быть конечно представленными (и, более того, быть конечно порожденными) не является обязательным.

Пусть $N=|G|$ - порядок группы $G$ и $\mathcal{C}=\left\{C_{1}, \ldots, C_{m}\right\}$ - множество классов сопряженности в $G$ таких, что $O=\coprod C_{i}$. Для класса сопряженности $C \in \mathcal{C}$ обозначим через $n_{C}=n_{g}$ порядок элемента $g \in C$. В каждом классе $C \in \mathcal{C}$ выберем и зафиксируем элемент $g_{C} \in C$.

Очевидно, что необходимым условием для подполугруппы $S$ полугруппы $S_{O}$ быть конечно порожденной является следующее условие: образ $\tau(S)$ является конечно порожденной полугруппой, где $\tau: S_{O} \rightarrow \mathbb{Z}_{\geqslant 0}^{m}-$ гомоморфизм типа.

ТЕОРема 1.1. Полугруппа разложений на множители $S_{O, 1}$ над конечной группой $G$ является конечно определенной полугруппой. 
ДоказАтельство. Пусть $O=C_{1} \cup \cdots \cup C_{m}$ - разложение в объединение классов сопряженности, и пусть $\mathbf{1} \notin O$. Перенумеруем элементы множества $O=\left\{g_{1}, \ldots, g_{K}\right\}$ таким образом, что $g_{i}=g_{C_{i}}$ при $i=1, \ldots, m$.

Для любого $g \in O$ имеем $s_{g}=x_{g}^{n_{g}} \in S_{O, \mathbf{1}}$. Обозначим через $F=\left\{s_{1}, \ldots, s_{M}\right\}$ множество элементов полугруппы $S_{O, \mathbf{1}}$, длина которых не превосходит $K^{N}$, где $N=|G|$, и предположим также, что $s_{i}=s_{g_{i}}=x_{g_{i}}^{n_{g_{i}}}$ при $i \leqslant K$. Покажем, что элементы $s_{1}, \ldots, s_{M} \in F$ порождают полугруппу $S_{O, \mathbf{1}}$.

ЛЕмма 1.3. Элемент $s \in S_{O, 1}$ длинь $\ln (s)>K^{N}$ может быть записан в виде

$$
s=s_{i_{1}}^{n_{1}} \cdot \ldots \cdot s_{i_{l}}^{n_{l}} \cdot \bar{s}
$$

где $1 \leqslant i_{1} \leqslant \cdots \leqslant i_{l} \leqslant K$ и элемент $\bar{s} \in S_{O, 1}$ имеет длину $\ln (\bar{s}) \leqslant K^{N}$.

ДоказАтельство. Если $\ln (s)>K^{N}$, то в представлении элемента $s$ в виде произведения $x_{g_{1}} \cdot \ldots \cdot x_{g_{\ln (s)}}$ имеется по крайней мере $N$ совпадающих множителей $x_{g}$ для некоторого $g \in O$. Поскольку $n_{g} \leqslant N$, то, передвигая $n_{g}$ из этих множителей влево (с помощью соотношений (1.1)), мы получим, что $s=s_{g} \cdot s^{\prime}$, где $s^{\prime} \in S_{O, 1}$ такой, что $\ln \left(s^{\prime}\right)<\ln (s)$.

Из леммы 1.3 следует, что $S_{O, 1}$ порождается элементами $s \in S_{O, 1}$ длины $\ln (s) \leqslant K^{N}$, т. е. $S_{O, 1}$ является конечно порожденной полугруппой.

Чтобы показать, что полугруппа $S_{O, 1}$ конечно определена, разделим на части множество всех соотношений следующим образом. Первое множество $R_{1}$ состоит из соотношений вида

$$
s_{i} \cdot s_{j}=s_{j} \cdot s_{i}, \quad s_{i}, s_{j} \in F
$$

Обозначим через $\mathbf{k}=\left(k_{1}, \ldots, k_{M}\right)$ упорядоченный набор неотрицательных целых чисел и положим $s_{\mathbf{k}}=s_{1}^{k_{1}} \cdot \ldots \cdot s_{M}^{k_{M}}$. Поскольку множество соотношений $R_{1}$ уже определено, мы можем считать, что все остальные соотношения в $S_{O, 1}$, связывающие порождающие элементы $s_{1}, \ldots, s_{M}$, имеют следующий вид:

$$
s_{\mathbf{k}_{1}}=s_{\mathbf{k}_{2}} \text {. }
$$

Заметим, что если мы имеем соотношение вида $(1.10)$, то $G_{s_{\mathbf{k}_{1}}}=G_{s_{\mathbf{k}_{2}}}$ и $\tau\left(s_{\mathbf{k}_{1}}\right)=\tau\left(s_{\mathbf{k}_{2}}\right)$.

Рассмотрим множество $\bar{R}_{2}$, состоящее из всех соотношений вида $(1.10)$, для которых группы $G_{s_{\mathbf{k}_{1}}}$ являются собственными подгруппами в $G$. По индукции мы можем считать, что полугруппы $S(\Gamma, \bar{O})_{1}$ являются конечно представимыми для всех оснащенных групп $(\Gamma, \bar{O})$, порядок которых меньше $N$. Поскольку в группе $G$ имеется лишь конечное число различных собственных подгрупп и вложения $\left(G_{s_{\mathbf{k}_{1}}}, O \cap G_{s_{\mathbf{k}_{1}}}\right) \hookrightarrow(G, O)$ определяют вложения $S\left(G_{s_{\mathbf{k}_{1}}}, O \cap G_{s_{\mathbf{k}_{1}}}\right)_{1} \hookrightarrow$ $S_{O, \mathbf{1}}$, отсюда вытекает, что имеется конечное множество соотношений $R_{2} \subset \bar{R}_{2}$ таких, что все соотношения $\bar{R}_{2}$ являются следствиями соотношений из $R_{1} \cup R_{2}$.

Обозначим через $R_{3}$ множество всех соотношений в $S_{O, \mathbf{1}}$ вида $s_{\mathbf{k}_{1}}=s_{\mathbf{k}_{\mathbf{2}}}$, которые не содержатся в $R_{1} \cup R_{2}$ и являются такими, что $\ln \left(s_{\mathbf{k}_{1}}\right) \leqslant K^{N}$. Очевидно, $R_{3}$ является конечным множеством. 
Для каждого элемента $s_{i}$ из множества порождающих элементов полугруппы $S_{O, \mathbf{1}}$ с номером $i \geqslant K+1$ положим

$$
n_{i}=\min _{n}\left\{\ln \left(s_{i}^{n}\right)>K^{N}\right\}-1 .
$$

Следующая лемма является следствием леммы 1.3 .

ЛЕмма 1.4. Для любого $i \geqslant K+1$ элемент $s_{i}^{n_{i}+1}$ может быть записан в виде

$$
s_{i}^{n_{i}+1}=\left(\prod_{j=1}^{K} s_{j}^{a_{j}}\right) \cdot s_{l},
$$

где $\mathbf{a}=\left(a_{1}, \ldots, a_{K}\right)$ - набор неотрицательных цельх чисел и $s_{l} \in F$ - некоторый порождающий элемент с номером $l \geqslant K+1$.

Обозначим через $R_{4}$ множество всех соотношений вида (1.11). Это конечное множество. Согласно лемме 1.4, применив соотношения из $R_{1} \cup R_{4}$, каждый элемент $s \in S_{O, \mathbf{1}}$ мы можем записать в виде $s=s_{\mathbf{k}}$, где набор $\mathbf{k}=\left(k_{1}, \ldots, k_{M}\right)$ удовлетворяет условию $k_{i} \leqslant n_{i}$ для $i \geqslant K+1$.

Элемент $s_{\mathbf{k}}$ называется Г-примитивным, если в наборе $\mathbf{k}=\left(k_{1}, \ldots, k_{M}\right)$ все $k_{i} \leqslant 1$ при $i \leqslant K, k_{i} \leqslant n_{i}$ при $i \geqslant K+1$ и $G_{s_{\mathrm{k}}}=\Gamma$. По лемме 1.1 для каждого $G$-примитивного элемента $s_{\mathbf{k}}$ имеют место следующие соотношения в $S_{O, 1}$ :

$$
s_{i} \cdot s_{\mathbf{k}}=s_{j} \cdot s_{\mathbf{k}}
$$

где $i \leqslant m$ и $j \leqslant K$ таковы, что $g_{j} \in C_{i}$. Обозначим через $R_{5}$ множество всех таких соотношений. Очевидно, $R_{5}$ является конечным множеством.

Пусть $s \in S_{O, 1}$ - элемент, группа которого $G_{s}=G$. Применив соотношения из $R_{5}$, как и выше, легко показать, что $s$ может быть записан в виде

$$
s=\left(\prod_{j=1}^{m} s_{j}^{a_{j}}\right) \cdot s_{\mathbf{k}},
$$

где $s_{\mathbf{k}}$ - некоторый $G$-примитивный элемент. Обозначим через $\bar{R}_{6}$ множество всех соотношений в $S_{O, 1}$ вида

$$
\left(\prod_{j=1}^{m} s_{j}^{b_{j, 1}}\right) \cdot s_{\mathbf{k}_{1}}=\left(\prod_{j=1}^{m} s_{j}^{b_{j, 2}}\right) \cdot s_{\mathbf{k}_{2}},
$$

где $s_{\mathbf{k}_{1}}$ и $s_{\mathbf{k}_{2}}-G$-примитивные элементы.

Чтобы завершить доказательство теоремы, достаточно показать, что все соотношения из множества $\bar{R}_{6}$ являются следствиями из конечного множества соотношений $R_{6}$. Поскольку существует только конечное число $G$-примитивных элементов, для этого достаточно показать, что для фиксированных $G$-примитивных элементов $s_{\mathbf{k}_{1}}$ и $s_{\mathbf{k}_{2}}$ соотношения вида $(1.13)$ являются следствиями некоторого конечного множества соотношений.

Заметим, что если мы имеем соотношение вида (1.13), то

$$
\left(b_{1,1} n_{C_{1}}, \ldots, b_{m, 1} n_{C_{m}}\right)+\tau\left(s_{\mathbf{k}_{1}}\right)=\left(b_{1,2} n_{C_{1}}, \ldots, b_{m, 2} n_{C_{m}}\right)+\tau\left(s_{\mathbf{k}_{2}}\right) .
$$


Следовательно, если $\tau\left(s_{\mathbf{k}_{j}}\right)=\left(\alpha_{1, j}, \ldots, \alpha_{m, j}\right)$, то $\alpha_{i, 1} \equiv \alpha_{i, 2}\left(\bmod n_{C_{i}}\right)$ при всех $i$. Положим $a_{i, 1,0}=b_{i, 1}-b_{i, 2}$, если $\alpha_{i, 2} \geqslant \alpha_{i, 1}$, и $a_{i, 1,0}=0$ в противном случае. Соответственно, положим $a_{i, 2,0}=b_{i, 2}-b_{i, 1}$, если $\alpha_{i, 1} \geqslant \alpha_{i, 2}$, и $a_{i, 2,0}=0$ в противном случае. Имеем

$$
n_{C_{i}} a_{i, 1,0}+\alpha_{i, 1}=n_{C_{i}} a_{i, 2,0}+\alpha_{i, 2},
$$

и числа $a_{i, 1,0}, a_{i, 2,0}$ определены однозначно числами $\alpha_{i, 1}, \alpha_{i, 2}$ и $n_{C_{i}}$. Более того, если мы обозначим $a_{i, j}=b_{i, j}-a_{i, j, 0}$, то $a_{i, 1}=a_{i, 2} \geqslant 0$ для $i=1, \ldots, m$ и каждое соотношение вида (1.13) может быть записано следующим образом:

$$
\left(\prod_{j=1}^{m} s_{j}^{a_{j}}\right) \cdot\left(\prod_{j=1}^{m} s_{j}^{a_{j, 1,0}}\right) \cdot s_{\mathbf{k}_{1}}=\left(\prod_{j=1}^{m} s_{j}^{a_{j}}\right) \cdot\left(\prod_{j=1}^{m} s_{j}^{a_{j, 2,0}}\right) \cdot s_{\mathbf{k}_{2}},
$$

где $a_{j}=a_{j, 1}=a_{j, 2}$.

Если (1.14) является соотношением в полугруппе $S_{O, \mathbf{1}}$, то

$$
\left(\prod_{j=1}^{m} s_{j}^{a_{j}+b_{j}}\right) \cdot\left(\prod_{j=1}^{m} s_{j}^{a_{j, 1,0}}\right) \cdot s_{\mathbf{k}_{1}}=\left(\prod_{j=1}^{m} s_{j}^{a_{j}+b_{j}}\right) \cdot\left(\prod_{j=1}^{m} s_{j}^{a_{j, 2,0}}\right) \cdot s_{\mathbf{k}_{2}}
$$

также является соотношением для каждого набора $\mathbf{b}=\left(b_{1}, \ldots, b_{m}\right) \in \mathbb{Z}_{\geqslant 0}^{m}$ и оно является следствием соотношения (1.14).

Из изложенного выше следует, что множество экспонент $\left\{\left(a_{1}, \ldots, a_{m}\right)\right\}$, входящих в соотношения вида (1.14) при фиксированных $s_{\mathbf{k}_{1}}$ и $s_{\mathbf{k}_{2}}$, образует неперфорированную подполугруппу $F_{s_{\mathbf{k}_{1}}, s_{\mathbf{k}_{2}}}$ в полугруппе $\mathbb{Z}_{\geqslant 0}^{m}$. Согласно следствию 1.1 множество $O\left(F_{s_{\mathbf{k}_{1}}, s_{\mathbf{k}_{2}}}\right)$ его вершин конечно. Легко видеть, что соотношения вида (1.14) при фиксированных $s_{\mathbf{k}_{1}}$ и $s_{\mathbf{k}_{2}}$ являются следствиями соотношений, соответствующих вершинам подполугруппы $F_{s_{\mathbf{k}_{1}}, s_{\mathbf{k}_{2}}}$, и так как имеется только конечное множество $G$-примитивных элементов, то мы получаем, что соотношения из множества $\bar{R}_{6}$ являются следствиями некоторого конечного подмножества соотношений $R_{6} \subset \bar{R}_{6}$.

Для завершения доказательства теоремы достаточно заметить, что все соотношения являются следствиями соотношений, принадлежащих конечному множеству $R_{1} \cup \cdots \cup R_{6}$. Теорема доказана.

Отметим, что не любая подполугруппа $S_{O, 1}^{G}$ полугруппы $S_{G}$ является конечно порожденной. Например, группа $G \simeq(\mathbb{Z} / 2 \mathbb{Z})^{2}$ порождается двумя элементами, скажем $g_{1}$ и $g_{2}$. Если $O=\left\{g_{1}, g_{2}\right\}$, то $S_{O, 1}^{G}$ изоморфна полугруппе

$$
S=\left\{\left(a_{1}, a_{2}\right) \in \mathbb{Z}_{\geqslant 0}^{2} \mid a_{1}>0, a_{2}>0\right\},
$$

которая не является конечно порожденной.

ПреДЛОЖеНИЕ 1.4. Пусть $(G, O)$ - конечная оснащенная группа. Предположим, что $O$ является объединением классов сопряженности: $O=C_{1} \cup \cdots$ $\cdots \cup C_{m}$, таким, что для каждого $i$ элементы, принадлежащие классу $C_{i}$, порождают всю группу $G$. Тогда подполугруппа $S_{O, 1}^{G}$ полугруппь $S_{G}$ является конечно определенной. 
ДокАЗАТЕЛьство. Используем те же обозначения, что и в доказательстве теоремы 1.1. Рассмотрим элемент

$$
s_{C_{i}}=\prod_{g_{l} \in C_{i}} x_{g_{l}}^{n_{C_{i}}}=\prod_{g_{l} \in C_{i}} s_{l} .
$$

Имеем $s_{C_{i}} \in S_{O, \mathbf{1}}^{G}$, так как элементы $g_{l} \in C_{i}$ порождают группу $G$.

Как было показано в доказательстве теоремы 1.1 , любой элемент $s \in S_{O, \mathbf{1}}^{G}$ может быть записан в виде (1.12):

$$
s=\left(\prod_{i=1}^{m} s_{i}^{a_{i}}\right) \cdot s_{\mathbf{k}},
$$

где $s_{\mathbf{k}}$ - некоторый $G$-примитивный элемент полугруппы $S_{O, \mathbf{1}}^{G}$. Если $a_{i} \geqslant\left|C_{i}\right|$, то согласно лемме 1.1

$$
s_{i}^{a_{i}} \cdot s_{\mathbf{k}}=s_{C_{i}} \cdot s_{i}^{a_{i}-\left|C_{i}\right|} \cdot s_{\mathbf{k}} \cdot
$$

Следовательно, любой элемент $s \in S_{O, 1}^{G}$ может быть записан в виде

$$
s=\left(\prod_{i=1}^{m} s_{C_{i}}^{b_{i}}\right) \cdot\left(\prod_{i=1}^{m} s_{i}^{a_{i}}\right) \cdot s_{\mathbf{k}},
$$

где $\left(b_{1}, \ldots, b_{k}\right) \in \mathbb{Z}_{\geqslant 0}^{k}$ и $0 \leqslant a_{i}<\left|C_{i}\right|$, а $s_{\mathbf{k}}$ - некоторый $G$-примитивный элемент. Отсюда следует, что $S_{O, 1}^{G}$ порождается элементами

$$
\left(\prod_{i=1}^{m} s_{i}^{a_{i}}\right) \cdot s_{\mathbf{k}}
$$

где $0 \leqslant a_{i}<\left|C_{i}\right|$, а $s_{\mathbf{k}}$ - некоторый $G$-примитивный элемент, и элементами $s_{C_{i}}$, $i=1, \ldots, m$. Очевидно, что это множество порождающих элементов является конечным. Чтобы доказать конечную определенность полугруппы $S_{O, \mathbf{1}}^{G}$, заметим, что все соотношения между этими порождающими элементами являются следствиями соотношений коммутирования и множества соотношений, которое в доказательстве теоремы 1.1 обозначено через $\bar{R}_{6}$. Поэтому конец доказательства предложения совпадает с соответствующей частью доказательства теоремы 1.1.

1.6. Стабилизирующие элементы. Если $G$ - конечная абелева группа, то очевидно, что гомоморфизм типа $\tau: S_{G} \rightarrow \mathbb{Z}_{\geqslant 0}^{|G|-1}$ является изоморфизмом. Если $G$ не является абелевой группой и $c(G)$ - число классов сопряженности ее элементов $g \neq \mathbf{1}$, то гомоморфизм типа $\tau: S_{G} \rightarrow \mathbb{Z}_{\geqslant 0}^{c(G)}$ является сюръективным, но не инъективным гомоморфизмом и одна из основных проблем состоит в описании прообразов $\tau^{-1}(\mathbf{a})$ элементов $\mathbf{a} \in \mathbb{Z}_{\geqslant 0}^{c(G)}$ (в частности, описании множества элементов $\mathbf{a} \in \mathbb{Z}_{\geqslant 0}^{c(G)}$, для которых каждый элемент $s \in \tau^{-1}(\mathbf{a})$ однозначно определяется своим значением $\alpha(s) \in G)$.

ПРеДЛОЖЕНИЕ 1.5. Пусть полугруппа $S_{O, 1}^{G}$ такая же, как и в предложении 1.4. Тогда существует константа $c=c(G, O)$ такая, что для любого $\mathbf{a} \in \mathbb{Z}_{\geqslant 0}^{m}$ число $\left|\tau^{-1}(\mathbf{a})\right|$ прообразов элемента а при гомоморфизме $\tau: S_{O, \mathbf{1}}^{G} \rightarrow$ $\mathbb{Z}_{\geqslant 0}^{m}$ менъие $с$. 
ДокАЗАТЕЛЬСтво. В доказательстве предложения 1.4 было показано, что каждый элемент $s \in S_{O, 1}^{G}$ может быть записан в виде (1.15). Поэтому предложение следует из того, что число различных выражений вида (1.15), имеющих одинаковый тип, является конечным и ограничено некоторой константой $c$, не зависящей от типов этих выражений.

Отметим, что предложение 1.5 становится не верным, если мы рассмотрим полугруппу $S_{O, 1}$ вместо полугруппы $S_{O, 1}^{G}$ (см., например, следствие 2.4).

Элемент $s \in S(G, O)$ называется стабилизирующим, если $s \cdot s_{1}=s \cdot s_{2}$ для любых $s_{1}, s_{2} \in S(G, O)$ таких, что $\tau\left(s_{1}\right)=\tau\left(s_{2}\right)$ и $\alpha\left(s_{1}\right)=\alpha\left(s_{2}\right)$. Полугруппа $S(G, O)$ называется стабильной, если она обладает стабилизирующим элементом.

УТвЕРЖДЕНИЕ 1.8. Если $s$ - является стабилизирующим элементом полугруппы $S(G, O)$, то для любого $s_{1} \in S(G, O)$ элемент $s \cdot s_{1}$ также является стабилизируюшим. В частности, если $S(G, O)$ - стабильная полугруппа, то существует стабилизирующий элемент $s \in S(G, O)$ такой, что $\alpha(s)=1$.

\section{ДокАЗАТЕЛЬСТво очевидно.}

Теорема Конвея-Паркера (см. приложение в [5]) дает некоторое достаточное условие для того, чтобы полугруппа $S_{G}$ была стабильной. Чтобы сформулировать эту теорему, напомним, что накрывающей группой Шура $R$ конечной группы $G$ называется группа максимального порядка, обладающая свойством: $R$ имеет подгруппу $M \subset R^{\prime} \cap Z(R)$ такую, что $R / M \simeq G$, где $R^{\prime}=[R, R]-$ коммутант и $Z(R)$ - центр группы $R$. Такая группа $R$ существует всегда (но не обязательно, что такая группа единственна). Группа $M$ изоморфна мультипликатору Шура $M(G)=H^{2}\left(G, \mathbb{C}^{*}\right)$ группы $G$. Говорят, что мультипликатор Шура $M(G)$ порождается коммутаторами, если $M \cap\left\{g^{-1} h^{-1} g h \mid g, h \in R\right\}$ порождает группу $M$.

Теорема 1.2 (Дж. Х. Конвей, Р. А. Паркер [5]). Пусть G - конечная групnа, $O=G \backslash \mathbf{1}=C_{i} \cup \cdots \cup C_{m}$ - разложение в объединение классов сопряженности. Обозначим

$$
\bar{s}=\prod_{g \in G \backslash\{\mathbf{1}\}} x_{g}^{n_{g}} \in S_{G},
$$

где $n_{g}$ - порядок элемента $g$ в группе $G$. Предположим, что мультипликатор Шура $M(G)$ группы $G$ порождается коммутаторами. Тогда существует константа $n=n(G)$ такая, что $\bar{s}^{n}$ является стабилизирующим элементом полугруппь $S_{G}$.

Отметим, что накрывающая группа Шура $G$ конечной группы $H$ удовлетворяет условиям теоремы Конвея-Паркера (см. [5]).

В следующем параграфе мы докажем, что полугруппа разложений на множители $S_{\mathcal{S}_{d}}$ симметрической группы $\mathcal{S}_{d}$ также является стабильной. С другой стороны, имеется много конечных оснащенных групп $(G, O)$, для которых полугруппы $S(G, O)$ не являются стабильными.

ПРЕДЛОЖЕНИЕ 1.6. Пусть $(H, \widetilde{O})$ - конечная оснащенная группа такая, чmo: 
(i) элементы из $\widetilde{O}$ порождают группу $H$;

(ii) $H^{\prime} \cap Z(H) \neq \mathbf{1}$;

(iii) $\tilde{g}_{1} \tilde{g}_{2}^{-1} \notin Z(H) \backslash\{\mathbf{1}\}$ для всех $\tilde{g}_{1}, \tilde{g}_{2} \in \widetilde{O}$.

Пусть $f: H \rightarrow H / Z(H)=G$ - естественный эпиморфизм, и пусть $O=f(\widetilde{O}) \subset G$. Тогда существуют по крайней мере два элемента $s_{1}, s_{2} \in S_{O, 1}^{G}$ таких, что $\tau\left(s \cdot s_{1}\right)=\tau\left(s \cdot s_{2}\right)$, но $s \cdot s_{1} \neq s \cdot s_{2}$ для всех $s \in S_{O, \mathbf{1}}^{G} \cdot$ В частности, если $\widetilde{O}$ состоит только из одного класса сопряжснности группъ $H$, то существует константа $N \in \mathbb{N}$ такая, ито для любого $t \in \tau\left(S_{O, 1}^{G}\right) \cap \mathbb{Z}_{\geqslant N}$ существуют по крайней мере два элемента $s_{1}, s_{2} \in S_{O, 1}^{G} \operatorname{ma\kappa ux,~что~} \tau\left(s_{1}\right)=\tau\left(s_{2}\right)=t$, Ho $s_{1} \neq s_{2}$.

ДоказАТЕЛЬСтво. Согласно условию (i) элементы из $O$ порождают группу $G$. Из условия (iii) следует, что сюръективное отображение $f_{\mid \widetilde{O}}: \widetilde{O} \rightarrow O$ является биекцией, и если мы обозначим $g_{i}=f\left(\tilde{g}_{i}\right)$ для $\tilde{g}_{i} \in \widetilde{O}$, то равенство $g_{i}^{-1} g_{j} g_{i}=g_{k}$ выполнено в группе $G$ для некоторых $g_{i}, g_{j}, g_{k} \in O$ тогда и только тогда, когда равенство $\tilde{g}_{i}^{-1} \tilde{g}_{j} \tilde{g}_{i}=\tilde{g}_{k}$ выполнено в $H$. Следовательно, индуцированный гомоморфизм $f_{*}: S_{\widetilde{O}} \rightarrow S_{O}$ (отображающий порождающие элементы $x_{\tilde{g}_{i}}$ полугруппы $S_{\widetilde{O}}$ в порождающие элементы $x_{g_{i}}$ полугруппы $S_{O}$ ) является изоморфизмом полугрупп. В частности, ограничение отображения $f_{*}$ на $S_{\widetilde{O}, Z(H)}^{H}=\left\{\tilde{s} \in S_{O}^{H} \mid \alpha(\tilde{s}) \in Z(H)\right\}$ дает изоморфизм между $S_{\widetilde{O}, Z(H)}^{H}$ и $S_{O, \mathbf{1}}^{G}$. Кроме того, гомоморфизм $f$ индуцирует сюръективный гомоморфизм $f_{*}: \widehat{H}_{\widetilde{O}} \rightarrow \widehat{G}_{O} C$-групп, ассоциированных с оснащенными группами $(H, \widetilde{O})$ и $(G, O)\left(f_{*}\right.$ отображает порождающие элементы $y_{\tilde{g}_{i}}$ группы $\widehat{H}_{\widetilde{O}}$ в порождающие элементы $y_{g_{i}}$ группы $\left.\widehat{G}_{O}\right)$, такой, что диаграмма

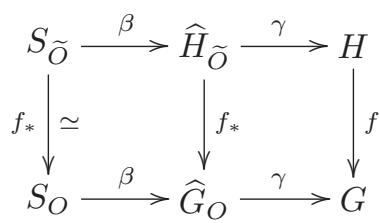

является коммутативной и, кроме того, индуцированный гомоморфизм

$$
f_{* *}: H_{1}\left(\widehat{H}_{\widetilde{O}}, \mathbb{Z}\right) \rightarrow H_{1}\left(\widehat{G}_{O}, \mathbb{Z}\right)
$$

является изоморфизмом, совместимым с изоморфизмом $f_{*}: S_{\widetilde{O}} \rightarrow S_{O}$ (т. е. если $s=f_{*}(\tilde{s})$, то $\left.\tau(s)=f_{* *}(\tau(\tilde{s}))\right)$. Следовательно, чтобы доказать первую часть предложения, достаточно показать, что существуют два элемента $\tilde{s}_{1}, \tilde{s}_{2} \in$ $S_{\widetilde{O}, Z(H)}^{H}$ таких, что $\tau\left(\tilde{s}_{1}\right)=\tau\left(\tilde{s}_{2}\right)$, но $\alpha\left(\tilde{s}_{1}\right) \neq \alpha\left(\tilde{s}_{2}\right)$. Действительно, для таких двух элементов имеем $\tau\left(\tilde{s} \cdot \tilde{s}_{1}\right)=\tau\left(\tilde{s} \cdot \tilde{s}_{2}\right)$, но $\alpha\left(\tilde{s} \cdot \tilde{s}_{1}\right) \neq \alpha\left(\tilde{s} \cdot \tilde{s}_{2}\right)$ для всех $\tilde{s} \in S_{\widetilde{O}, Z(H)}^{H}$. Следовательно, ввиду изоморфизма $f_{*}: S_{\widetilde{O}, Z(H)}^{H} \stackrel{\simeq}{\longrightarrow} S_{O, 1}^{G}$ элементы $s_{1}=f_{*}\left(\tilde{s}_{1}\right)$ и $s_{2}=f_{*}\left(\tilde{s}_{2}\right)$ не равны друг другу в полугруппе $S_{O, \mathbf{1}}$, но $\tau\left(s \cdot s_{1}\right)=\tau\left(s \cdot s_{2}\right)$ и $s \cdot s_{1} \neq s \cdot s_{2}$ для всех элементов $s \in S_{O, \mathbf{1}}^{G}$.

Из предложения 1.2 следует, что для любой подгруппы $\widehat{H}_{1}$ группы $\widehat{H}_{\widetilde{O}}$ имеем

$$
\gamma\left(\widehat{H}_{1} \cap Z\left(\widehat{H}_{\widetilde{O}}\right)\right)=\gamma\left(\widehat{H}_{1}\right) \cap Z(H),
$$


в частности

$$
\gamma\left(\widehat{H}_{\widetilde{O}}^{\prime} \cap Z\left(\widehat{H}_{\widetilde{O}}\right)\right)=H^{\prime} \cap Z(H)
$$

Поэтому согласно условию (ii) найдется элемент $\hat{h} \in \widehat{H}_{\widetilde{O}}^{\prime} \cap Z\left(\widehat{H}_{\widetilde{O}}\right) \backslash\{\mathbf{1}\}$. Согласно лемме 1.2 имеем $\hat{h}=\hat{h}_{1} \hat{h}_{2}^{-1}$, где $\hat{h}_{1}=\beta\left(\hat{s}_{1}\right)$ и $\hat{h}_{2}=\beta\left(\hat{s}_{2}\right)$ для некоторых $\hat{s}_{1}, \hat{s}_{2} \in$

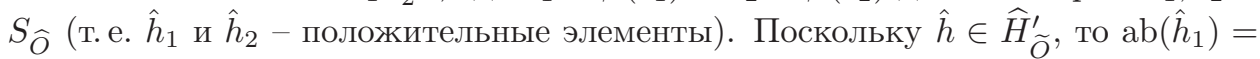
$\operatorname{ab}\left(\hat{h}_{2}\right)$.

Каждый элемент конечной группы $H$ может быть записан в виде некоторого положительного слова, состоящего из порождающих элементов. Следовательно, согласно условию (i) существуют $\hat{s} \in S_{\widetilde{O}}$ и положительный элемент $\hat{g}=\beta(\hat{s}) \in \widehat{H}_{\widetilde{O}}$ такие, что $\gamma(\hat{g})=\gamma\left(\hat{h}_{2}^{-1}\right)$. Обозначим $\hat{s}_{0}=\prod_{\tilde{g}_{i} \in \widetilde{O}} x_{\tilde{g}_{i}}^{n_{i}} \in S_{\widetilde{O}, \mathbf{1}}^{H}$, где $n_{i}$ - порядок элемента $\tilde{g}_{i}$. Тогда $\tilde{s}_{1}=\hat{s}_{0} \cdot \hat{s} \cdot \hat{s}_{1}$ и $\tilde{s}_{2}=\hat{s}_{0} \cdot \hat{s} \cdot \hat{s}_{2}$ являются искомыми элементами.

Чтобы доказать вторую часть предложения, выберем элементы $\bar{s}_{1}, \ldots, \bar{s}_{n}$, порождающие полугруппу $S_{O, 1}^{G}$ (согласно предложению 1.4 полугруппа $S_{O, 1}^{G}$ является конечно порожденной в случае, когда $O$ состоит из одного класса сопряженности), и пусть $s_{1}, s_{2}$ - два элемента, существование которых доказано в первой части доказательства. Обозначим $t_{0}=\tau\left(s_{1}\right)=\tau\left(s_{2}\right)$ и $t_{i}=\tau\left(\bar{s}_{i}\right)$ при $i=1, \ldots, n$, и пусть НОД $\left(t_{1}, \ldots, t_{n}\right)=d, t_{i}=a_{i} d$. Тогда тип $\tau(s)$ любого элемента полугруппы $S_{O, 1}^{G}$ делится на $d$. Покажем, что существует константа $M \in \mathbb{N}$ такая, что для любого $j \in \mathbb{N}$ найдется элемент $s \in S_{O, \mathbf{1}}^{G}$, тип которого есть $\tau(s)=(M+j) d$. Действительно, найдутся числа $q_{1}, \ldots, q_{n} \in \mathbb{Z}$ такие, что

$$
\sum_{i=1}^{n} q_{i} a_{i}=1
$$

После, быть может, перенумерации элементов $\bar{s}_{i}$ мы можем предполагать, что $q_{i}=-p_{i}<0$ при $i \leqslant k$ и $q_{i} \geqslant 0$ при $i \geqslant k+1$. Положим $M=a_{1} d \sum_{i=1}^{k} a_{i} p_{i}$ и для $j=0,1, \ldots, a_{1}$ рассмотрим элементы

$$
s_{0, j}=\left(\prod_{i=1}^{k} \bar{s}_{i}^{\left(a_{1}-j\right) p_{i}}\right) \cdot\left(\prod_{i=k+1}^{n} \bar{s}_{i}^{j q_{i}}\right) \in S_{O, \mathbf{1}}^{G} .
$$

Имеем

$$
\tau\left(s_{0, j}\right)=d a_{1} \sum_{i=1}^{k} p_{i} a_{i}+d j\left(-\sum_{i=1}^{k} a_{i} p_{i}+\sum_{i=k+1}^{n} a_{i} q_{i}\right)=d(M+j)
$$

для $0 \leqslant j \leqslant a_{1}$. Поэтому $\tau\left(\bar{s}_{1}^{m} \cdot s_{0, j}\right)=d\left(m a_{1}+M+j\right)$. Поскольку

$$
\left\{d\left(m a_{1}+M+j\right) \mid m \geqslant 0,0 \leqslant j \leqslant a_{1}\right\}=d \mathbb{N}_{\geqslant M},
$$

отсюда легко видеть, что $M$ удовлетворяет условию: для любого $j \in \mathbb{N}$ найдется элемент $s \in S_{O, \mathbf{1}}^{G}$, тип которого есть $\tau(s)=(M+j) d$.

Для завершения доказательства предложения осталось заметить, что $N=$ $M+t_{0}=M+\tau\left(s_{1}\right)$ является искомой константой. 
Несложно дать примеры групп $H$, удовлетворяющих условиям предложения 1.6. Например, пусть $H=\mathrm{SL}_{p-1}\left(\mathbb{Z}_{p}\right)$ - группа $((p-1) \times(p-1))$-матриц с определителем 1 над конечным полем $\mathbb{Z}_{p}, p \neq 2$. Хорошо известно, что $H^{\prime}=H$, а центр $Z(H)$, состоящий из скалярных матриц, является циклической группой порядка $p-1$. Для $i \neq j$ обозначим через $e_{i, j}$ матрицу, все элементы которой равны нулю, за исключением одного, равного единице и стоящего на пересечении $i$-й строки и $j$-го столбца. Положим $t_{i, j}=e+e_{i, j}$, где $e$ - единичная матрица. Хорошо известно, что матрицы $t_{i, j}$ (так называемые трансвекции) сопряжены друг другу и порождают группу $H=\mathrm{SL}_{p-1}\left(\mathbb{Z}_{p}\right)$. Следовательно, для оснащенной группы $(G, O)$, где $G=\mathrm{PGL}_{p-1}\left(\mathbb{Z}_{p}\right)$ и $O$ - множество трансвекций, почти все элементы полугруппы $S_{O, 1}^{G}$ не определяются однозначно своим типом, другими словами, $S_{O, 1}^{G}$ (соответственно, $\left.S_{O}\right)$ не является стабильной полугруппой.

\section{§ 2. Полугруппы разложений на множители в симметрической группе}

2.1. Основные обозначения и определения. Пусть $\mathcal{S}_{d}$ - симметрическая группа, действующая на множестве $\{1, \ldots, d\}=I_{d}$. Напомним, что элемент $\sigma=\left(i_{1}, \ldots, i_{k}\right) \in \mathcal{S}_{d}$, отображающий $i_{1}$ в $i_{2}, i_{2}$ в $i_{3}, \ldots, i_{k-1}$ в $i_{k}$, $i_{k}$ в $i_{1}$ и оставляющий не месте все остальные элементы множества $I_{d}$, называется ииклической перестановкой длины $k$. Циклическая перестановка длины 2 называется транспозицией. Любая циклическая перестановка $\sigma=\left(i_{1}, \ldots, i_{k}\right)$ является произведением $k-1$ транспозиций:

$$
\sigma=\left(i_{1}, i_{2}\right)\left(i_{2}, i_{3}\right) \ldots\left(i_{k-1}, i_{k}\right)
$$

Разложение $(2.1)$ циклической перестановки $\sigma=\left(i_{1}, \ldots, i_{k}\right)$ называется каноническим, если $i_{1}=\min _{1 \leqslant j \leqslant k} i_{j}$.

Как известно, любая перестановка $\sigma \in \mathcal{S}_{d}, \sigma \neq \mathbf{1}$, может быть представлена в виде произведения циклических перестановок:

$$
\sigma=\left(i_{1,1}, \ldots, i_{k_{1}, 1}\right)\left(i_{1,2}, \ldots, i_{k_{2}, 2}\right) \ldots\left(i_{1, m}, \ldots, i_{k_{m}, m}\right),
$$

где $k_{1} \geqslant k_{2} \geqslant \cdots \geqslant k_{m} \geqslant 2$ и при $j_{1} \neq j_{2}$ любые два множества $\left\{i_{1, j_{1}}, \ldots, i_{k_{j_{1}}, j_{1}}\right\}$ и $\left\{i_{1, j_{2}}, \ldots, i_{k_{j_{2}}, j_{2}}\right\}$ имеют пустое пересечение. Если перестановка $\sigma$ записана в виде $(2.2)$, то упорядоченный набор $t(\sigma)=\left[k_{1}, \ldots, k_{m}\right]$ называется типом перестановки $\sigma$, а число $l_{t}(\sigma)=\sum_{i=1}^{m} k_{i}-m$ называется ее транспозиционной длиной.

Отметим, что для любых $k_{1} \geqslant k_{2} \geqslant \cdots \geqslant k_{m} \geqslant 2$ таких, что $\sum k_{j} \leqslant d$, существует перестановка $\sigma$, тип которой есть $\left[k_{1}, \ldots, k_{m}\right]$, и, как хорошо известно, две перестановки $\sigma_{1}$ и $\sigma_{2}$ сопряжены в $\mathcal{S}_{d}$ тогда и только тогда, когда $t\left(\sigma_{1}\right)=t\left(\sigma_{2}\right)$. Для фиксированного типа $t(\sigma)=\left[k_{1}, \ldots, k_{m}\right]$ перестановка

$$
\left(1, \ldots, k_{1}\right)\left(k_{1}+1, \ldots, k_{1}+k_{2}\right) \ldots\left(\sum_{i=1}^{m-1} k_{i}+1, \ldots, \sum_{i=1}^{m} k_{i}\right)
$$

называется каноническим представителем типа $t(\sigma)$. Скажем, что mun $t\left(\sigma_{1}\right)=$ $\left[k_{1,1}, \ldots, k_{m_{1}, 1}\right]$ больше, чем тип $t\left(\sigma_{2}\right)=\left[k_{1,2}, \ldots, k_{m_{2}, 2}\right]$, если существует $l \geqslant 0$ 
такое, что $k_{1, i}=k_{2, i}$ при $i \leqslant l$ и $k_{1, l+1}>k_{2, l+1}$ (здесь $k_{j, i}=0$, если $i>m_{j}$ ). Скажем, что ииклическая перестановка $\sigma_{1}=\left(j_{1}, \ldots, j_{k_{1}}\right)$ больше, чем ииклическая перестановка $\sigma_{2}=\left(l_{1}, \ldots, l_{k_{2}}\right)$, если $t\left(\sigma_{1}\right) \geqslant t\left(\sigma_{2}\right)$, причем если $t\left(\sigma_{1}\right)=t\left(\sigma_{2}\right)$, то найдется $r<k_{1}=k_{2}$ такое, что $j_{1}=l_{1}, \ldots, j_{r}=l_{r}$ и $j_{r+1}>l_{r+1}$ в канонических представлениях перестановок $\sigma_{1}$ и $\sigma_{2}$. Наконец, скажем, что перестановка $\sigma_{1}$ больше, чем перестановка $\sigma_{2}$, если $t\left(\sigma_{1}\right) \geqslant t\left(\sigma_{2}\right)$, причем если $t\left(\sigma_{1}\right)=t\left(\sigma_{2}\right)$ и $\sigma_{i}=\sigma_{i, 1} \ldots \sigma_{i, m}, i=1,2,-$ разложения в произведения циклических перестановок, то найдется $l$ такое, что $\sigma_{1, j}=\sigma_{2, j}$ при $j<l$ и $\sigma_{1, l}>\sigma_{2, l}$. Обозначим через $\mathcal{T}=\left\{t_{1}<t_{2}<\cdots<t_{N}\right\}$ множество всех типов перестановок $\sigma \in \mathcal{S}_{d}$.

Согласно определению полугруппа разложений на множители $\Sigma_{d}=S\left(\mathcal{S}_{d}, \mathcal{S}_{d}\right)$ в симметрической группе $\mathcal{S}_{d}$ порождается алфавитом $X=\left\{x_{\sigma} \mid \sigma \in \mathcal{S}_{d}\right\}$. Пусть $s=x_{\sigma_{1}} \cdot \ldots \cdot x_{\sigma_{n}}-$ элемент полугруппы $\Sigma_{d}$. Применив соотношения (1.1) и $(1.2)$, мы можем считать, что $t\left(\sigma_{1}\right) \leqslant \cdots \leqslant t\left(\sigma_{n}\right)$, и в этом случае сумма $\tau(s)=\sum_{i=1}^{N} a_{i} t_{i}$ является типом элемента $s$, где $a_{i}$ - число множителей $x_{\sigma_{j}}$, входящих в $s$, для которых $t\left(\sigma_{j}\right)=t_{i}$.

Для подгруппы $Г$ симметрической группы $\mathcal{S}_{d}$ обозначим $\Sigma_{d, \Gamma}=\left\{s \in \Sigma_{d} \mid\right.$ $\alpha(s) \in \Gamma\}$ и $\Sigma_{d}^{\Gamma}=\left\{s \in \Sigma_{d} \mid\left(\mathcal{S}_{d}\right)_{s}=\Gamma\right\}$.

Если $J \subset I_{d}$ - подмножество множества $I_{d},|J|=d_{1} \leqslant d$, то вложение $J \subset I_{d}$ определяет вложение $\mathcal{S}_{d_{1}} \subset \mathcal{S}_{d}$ и вложение $\psi_{J}: \Sigma_{d_{1}} \hookrightarrow \Sigma_{d}$.

2.2. Разложения в произведения транспозиций. Обозначим через $T_{d}$ множество транспозиций группы $\mathcal{S}_{d}$. Подполугруппа $S_{T_{d}}$ полугруппы $\Sigma_{d}$ порождается элементами $x_{(i, j)}, 1 \leqslant i, j \leqslant d, i \neq j$, связанными соотношениями

$$
\begin{gathered}
x_{(i, j)}=x_{(j, i)} \quad \forall\{i, j\}_{\text {ord }} \subset I_{d}, \\
x_{\left(i_{1}, i_{2}\right)} \cdot x_{\left(i_{1}, i_{3}\right)}=x_{\left(i_{2}, i_{3}\right)} \cdot x_{\left(i_{1}, i_{2}\right)}=x_{\left(i_{1}, i_{3}\right)} \cdot x_{\left(i_{2}, i_{3}\right)} \quad \forall\left\{i_{1}, i_{2}, i_{3}\right\}_{\text {ord }} \subset I_{d}, \\
x_{\left(i_{1}, i_{2}\right)} \cdot x_{\left(i_{3}, i_{4}\right)}=x_{\left(i_{3}, i_{4}\right)} \cdot x_{\left(i_{1}, i_{2}\right)} \quad \forall\left\{i_{1}, i_{2}, i_{3}, i_{4}\right\}_{\text {ord }} \subset I_{d}
\end{gathered}
$$

(здесь $\left\{i_{1}, \ldots, i_{k}\right\}$ ord - подмножество множества $I_{d}$, состоящее из $k$ упорядоченных элементов; таким образом, для любого подмножества $\left\{i_{1}, \ldots, i_{k}\right\}$ множества $I_{d}$ мы имеем $k$ ! упорядоченных подмножеств $\left.\left\{\sigma\left(i_{1}\right), \ldots, \sigma\left(i_{k}\right)\right\}_{\text {ord }}, \sigma \in \mathcal{S}_{k}\right)$.

Обозначим $S_{T_{d}, \mathbf{1}}=S_{T_{d}} \cap \Sigma_{d, \mathbf{1}}$. Согласно предложению $\left.1.1,4\right)$ полугруппа $\Sigma_{d, 1}$ является подполугруппой центра полугруппы $\Sigma_{d}$. В частности, это коммутативная полугруппа.

Легко видеть, что для каждого подмножества $\{i, j\} \subset I_{d}$ элемент $s_{(i, j)}=$ $x_{i, j} \cdot x_{i, j}=x_{(i, j)}^{2}$ принадлежит полугруппе $S_{T_{d}, \mathbf{1}}$. Элемент

$$
h_{d, g}=s_{(1,2)}^{g+1} \cdot s_{(2,3)} \cdot \ldots \cdot s_{(d-1, d)} \in S_{T_{d}, 1} \subset \Sigma_{d}
$$

называется элементом Гурвица рода $\mathrm{g}$.

Лемма 2.1. Для любого упорядоченного подмножества $\left\{j_{1}, \ldots, j_{k+1}\right\}$ ord $\subset$ $I_{d}$ и любого $i, 1 \leqslant i \leqslant k$, элемент

$$
s=x_{\left(j_{1}, j_{2}\right)} \cdot x_{\left(j_{2}, j_{3}\right)} \cdot \ldots \cdot x_{\left(j_{k-1}, j_{k}\right)} \cdot x_{\left(j_{i}, j_{k+1}\right)} \in S_{T_{d}}
$$

равен элементу

$$
s_{i}=x_{\left(j_{1}, j_{2}\right)} \cdot \ldots \cdot x_{\left(j_{i-1}, j_{i}\right)} \cdot x_{\left(j_{i}, j_{k+1}\right)} \cdot x_{\left(j_{k+1}, j_{i+1}\right)} \cdot x_{\left(j_{i+1}, j_{i+2}\right)} \cdot \ldots \cdot x_{\left(j_{k-1}, j_{k}\right)} \cdot
$$


ДокАзАтельство. Согласно соотношениям (2.3) имеем (на каждом шаге преобразований подчеркивание означает, что будем преобразовывать подчеркнутые множители, а результат преобразования записывать в скобках)

$$
\begin{aligned}
s & =x_{\left(j_{1}, j_{2}\right)} \cdot x_{\left(j_{2}, j_{3}\right)} \cdot \ldots \cdot \frac{x_{\left(j_{i+1}, j_{i+2}\right)} \cdot \ldots \cdot x_{\left(j_{k-1}, j_{k}\right)} \cdot x_{\left(j_{i}, j_{k+1}\right)}}{} \\
& =x_{\left(j_{1}, j_{2}\right)} \cdot \ldots \cdot \frac{x_{\left(j_{i}, j_{i+1}\right)} \cdot\left(x_{\left(j_{i}, j_{k+1}\right)} \cdot x_{\left(j_{i+1}, j_{i+2}\right)} \cdot \ldots \cdot x_{\left(j_{k-1}, j_{k}\right)}\right)}{} \\
& =x_{\left(j_{1}, j_{2}\right)} \cdot \ldots \cdot x_{\left(j_{i-1}, j_{i}\right)}\left(\frac{\left(x_{\left(j_{i+1}, j_{k+1}\right)} \cdot x_{\left(j_{i}, j_{i+1}\right)}\right)}{} \cdot \ldots \cdot x_{\left(j_{k-1}, j_{k}\right)}\right. \\
& =x_{\left(j_{1}, j_{2}\right)} \cdot \ldots \cdot x_{\left(j_{i-1}, j_{i}\right)} \cdot\left(x_{\left(j_{i}, j_{k+1}\right)} \cdot x_{\left(j_{k+1}, j_{i+1}\right)}\right) \cdot x_{\left(j_{i+1}, j_{i+2}\right)} \cdot \ldots \cdot x_{\left(j_{k-1}, j_{k}\right)} .
\end{aligned}
$$

Лемма доказана.

ЛЕмма 2.2. Для любого упорядоченного подмножества $\left\{j_{1}, \ldots, j_{k}\right\}_{\text {ord }} \subset I_{d}$ и для любого $i, 1 \leqslant i \leqslant k$, элемент $s=x_{\left(j_{1}, j_{2}\right)} \cdot x_{\left(j_{2}, j_{3}\right)} \cdot \ldots \cdot x_{\left(j_{k-1}, j_{k}\right)} \cdot x_{\left(j_{i}, j_{k}\right)} \in S_{T_{d}}$, где $k \leqslant d-1$, равен элементу

$$
s_{i}=x_{\left(j_{1}, j_{2}\right)} \cdot \ldots \cdot x_{\left(j_{i-1}, j_{i}\right)} \cdot x_{\left(j_{i+1}, j_{i+2}\right)} \cdot \ldots \cdot x_{\left(j_{k-1}, j_{k}\right)} \cdot x_{\left(j_{i}, j_{i+1}\right)}^{2} .
$$

ДокАЗАТЕЛЬство. Согласно соотношениям (2.3) имеем

$$
\begin{aligned}
& s=x_{\left(j_{1}, j_{2}\right)} \cdot x_{\left(j_{2}, j_{3}\right)} \cdot \ldots \cdot \underline{x_{\left(j_{k-1}, j_{k}\right)} \cdot x_{\left(j_{i}, j_{k}\right)}} \\
& \left.=x_{\left(j_{1}, j_{2}\right)} \cdot x_{\left(j_{2}, j_{3}\right)} \cdot \ldots \cdot \underline{x_{\left(j_{k-2}, j_{k-1}\right)} \cdot\left(x_{\left(j_{i}, j_{k-1}\right)}\right.} \cdot x_{\left(j_{k-1}, j_{k}\right)}\right)=\cdots \\
& \cdots=x_{\left(j_{1}, j_{2}\right)} \cdot \ldots \cdot x_{\left(j_{i-1}, j_{i}\right)} \cdot x_{\left(j_{i}, j_{i+1}\right)} \cdot\left(x_{\left(j_{i}, j_{i+1}\right)} \cdot x_{\left(j_{i+1}, j_{i+2}\right)}\right) \cdot \ldots \cdot x_{\left(j_{k-1}, j_{k}\right)} \\
& =x_{\left(j_{1}, j_{2}\right)} \cdot \ldots \cdot x_{\left(j_{i-1}, j_{i}\right)} \cdot \underline{x_{\left(j_{i}, j_{i+1}\right)}^{2} \cdot x_{\left(j_{i+1}, j_{i+2}\right)}} \cdot \ldots \cdot x_{\left(j_{k-1}, j_{k}\right)} \\
& =x_{\left(j_{1}, j_{2}\right)} \cdot \ldots \cdot x_{\left(j_{i-1}, j_{i}\right)} \cdot\left(x_{\left(j_{i+1}, j_{i+2}\right)} \cdot \underline{\left.x_{\left(j_{i}, j_{i+1}\right)}^{2}\right) \cdot x_{\left(j_{i+2}, j_{i+3}\right)}} \cdot \ldots \cdot x_{\left(j_{k-1}, j_{k}\right)}=\cdots\right. \\
& \cdots=x_{\left(j_{1}, j_{2}\right)} \cdot \ldots \cdot x_{\left(j_{i-1}, j_{i}\right)} \cdot x_{\left(j_{i+1}, j_{i+2}\right)} \cdot \ldots \cdot\left(x_{\left(j_{k-1}, j_{k}\right)} \cdot x_{\left(j_{i}, j_{i+1}\right)}^{2}\right)=s_{i} .
\end{aligned}
$$

Лемма доказана.

Лемма 2.3. Равенства

$$
\begin{gathered}
x_{\left(i_{1}, i_{2}\right)}^{2} \cdot x_{\left(i_{2}, i_{3}\right)}=x_{\left(i_{2}, i_{3}\right)} \cdot x_{\left(i_{1}, i_{3}\right)}^{2}=x_{\left(i_{1}, i_{3}\right)}^{2} \cdot x_{\left(i_{2}, i_{3}\right)}=x_{\left(i_{2}, i_{3}\right)} \cdot x_{\left(i_{1}, i_{2}\right)}^{2}, \\
x_{\left(i_{1}, i_{2}\right)}^{2} \cdot x_{\left(i_{2}, i_{3}\right)}^{2}=x_{\left(i_{1}, i_{2}\right)}^{2} \cdot x_{\left(i_{1}, i_{3}\right)}^{2}=x_{\left(i_{2}, i_{3}\right)}^{2} \cdot x_{\left(i_{1}, i_{3}\right)}^{2}
\end{gathered}
$$

выполнены для всех упорядоченных троек $\left\{i_{1}, i_{2}, i_{3}\right\}_{\text {ord }} \subset I_{d}$, а равенства

$$
x_{\left(i_{1}, i_{2}\right)}^{2} \cdot x_{\left(i_{3}, i_{4}\right)}^{2}=x_{\left(i_{3}, i_{4}\right)}^{2} \cdot x_{\left(i_{1}, i_{2}\right)}^{2}
$$

выполнены для всех упорядоченных четверок $\left\{i_{1}, i_{2}, i_{3}, i_{4}\right\}_{\text {ord }} \subset I_{d}$.

ДокАзАтЕльство. Мы проверим только два из трех равенств (2.4), так как проверка остальных равенств аналогична. Согласно соотношениям (2.3) имеем

$$
\begin{aligned}
x_{\left(i_{1}, i_{2}\right)}^{2} \cdot x_{\left(i_{2}, i_{3}\right)} & =x_{\left(i_{1}, i_{2}\right)} \cdot \frac{x_{\left(i_{1}, i_{2}\right)} \cdot x_{\left(i_{2}, i_{3}\right)}}{=}=\frac{x_{\left(i_{1}, i_{2}\right)} \cdot\left(x_{\left(i_{2}, i_{3}\right)} \cdot x_{\left(i_{1}, i_{3}\right)}\right)}{}=\left(x_{\left(i_{2}, i_{3}\right)} \cdot x_{\left(i_{1}, i_{3}\right)}\right) \cdot x_{\left(i_{1}, i_{3}\right)}=x_{\left(i_{2}, i_{3}\right)} \cdot x_{\left(i_{1}, i_{3}\right)}^{2} .
\end{aligned}
$$

Аналогично,

$$
\begin{aligned}
x_{\left(i_{1}, i_{2}\right)}^{2} \cdot x_{\left(i_{2}, i_{3}\right)} & =x_{\left(i_{1}, i_{2}\right)} \cdot \underline{x_{\left(i_{1}, i_{2}\right)} \cdot x_{\left(i_{2}, i_{3}\right)}}=\frac{x_{\left(i_{1}, i_{2}\right)} \cdot\left(x_{\left(i_{1}, i_{3}\right)} \cdot x_{\left(i_{1}, i_{2}\right)}\right)}{}=\left(x_{\left(i_{2}, i_{3}\right)} \cdot x_{\left(i_{1}, i_{2}\right)}\right) \cdot x_{\left(i_{1}, i_{2}\right)}=x_{\left(i_{2}, i_{3}\right)} \cdot x_{\left(i_{1}, i_{2}\right)}^{2} .
\end{aligned}
$$

Лемма доказана. 
Следующая лемма является непосредственным следствием леммы 2.3 .

ЛЕмма 2.4. Для любого упорядоченного подмножества $\left\{j_{1}, \ldots, j_{k+1}\right\}$ ord $\subset$ $I_{d}$ и любого $i, 1 \leqslant i \leqslant k$, элемент $s_{i}=x_{\left(j_{1}, j_{2}\right)} \cdot x_{\left(j_{2}, j_{3}\right)} \cdot \ldots \cdot x_{\left(j_{k-1}, j_{k}\right)} \cdot x_{\left(j_{i}, j_{k+1}\right)}^{2} \in S_{T_{d}}$ равен элементу $s_{1}=x_{\left(j_{1}, j_{2}\right)} \cdot x_{\left(j_{2}, j_{3}\right)} \cdot \ldots \cdot x_{\left(j_{k-1}, j_{k}\right)} \cdot x_{\left(j_{1}, j_{k+1}\right)}^{2} \cdot$

Следующая лемма является частным случаем леммы 1.1.

Лемма 2.5. Для любого упорядоченного подмножества $\left\{j_{1}, \ldots, j_{k}\right\}$ ord $\subset I_{d}$ выполнено равенство

$$
x_{\left(j_{1}, j_{2}\right)}^{2} \cdot x_{\left(j_{1}, j_{2}\right)} \cdot x_{\left(j_{2}, j_{3}\right)} \cdot \ldots \cdot x_{\left(j_{k-1}, j_{k}\right)}=x_{\left(j_{i}, j_{l}\right)}^{2} \cdot x_{\left(j_{1}, j_{2}\right)} \cdot x_{\left(j_{2}, j_{3}\right)} \cdot \ldots \cdot x_{\left(j_{k-1}, j_{k}\right)},
$$

где $1 \leqslant i<l \leqslant k$.

Сопоставим каждому слову $w\left(\overline{x_{(i, j)}}\right)=x_{\left(i_{1}, j_{1}\right)} \ldots x_{\left(i_{m}, j_{m}\right)} \in W=W\left(T_{d}\right)$ граф $\widetilde{\Gamma}_{w}$, состоящий из $d$ вершин $v_{i}, 1 \leqslant i \leqslant d$, множество ребер которого находится во взаимно однозначном соответствии с набором букв, входящих в слово $w$, причем две вершины $v_{i}$ и $v_{j}$ соединены ребром, если буква $x_{(i, j)}$ содержится в $w$; в частности, число ребер, соединяющих вершины $v_{i}$ и $v_{j}$, равно числу вхождений буквы $x_{(i, j)}$ в $w$. Ребра графа $\widetilde{\Gamma}_{w}$ перенумерованы в соответствии с положением соответствующей буквы в слове $w$. Обозначим через $V_{\text {iso }}$ множество изолированных вершин графа $\widetilde{\Gamma}_{w}$ (т.е. вершина $v_{i}$ является изолированной, если она не соединена каким-нибудь ребром с другой вершиной графа $\left.\widetilde{\Gamma}_{w}\right)$ и положим $\Gamma_{w}=\widetilde{\Gamma}_{w} \backslash V_{\text {iso }}$.

Лемма 2.6. Пусть $w^{\prime}, w^{\prime \prime} \in W(s)=\{w \in W \mid \varphi(w)=s\}-$ два слова, представляющие элемент $s \in S_{T_{d}}, u$ пусть $\Gamma_{w^{\prime}}=\Gamma_{1,1} \sqcup \cdots \sqcup \Gamma_{1, n_{1}} u$ $\Gamma_{w^{\prime \prime}}=\Gamma_{2,1} \sqcup \cdots \sqcup \Gamma_{2, n_{2}}-$ представления графов $\Gamma_{w^{\prime}} u \Gamma_{w^{\prime \prime}}$ в виде несвязных обгединений своих связных компонент. Тогда $n_{1}=n_{2}:=n_{s}$ u существует взаимно однозначное соответствие между связными компонентами графов $\Gamma_{w^{\prime}} u \Gamma_{w^{\prime \prime}}$ mакое, что соответствующие графы $\Gamma_{1, i} u \Gamma_{2, i}, i=1, \ldots, n_{s}$, uмеют одно и то же множество вершин $V\left(\Gamma_{1, i}\right)=V\left(\Gamma_{2, i}\right):=V_{i}(s)$. Более того, элемент $s$ однозначно разлагается в произведение попарно коммутирующих множителей $s_{i} \in S_{T_{d}}, s=s_{1} \cdot \ldots \cdot s_{n_{s}}$, таких, что для каждого $i$ и для любого слова $w_{i} \in W\left(s_{i}\right)$ граф $\Gamma_{w_{i}}$ связен $u V\left(\Gamma_{w_{i}}\right)=V\left(\Gamma_{1, i}\right)$.

ДокАЗАТЕЛЬСТво непосредственно следует из соотношений (2.3).

ПреДЛОЖЕНИЕ 2.1. Пусть длина элемента $s \in S_{T_{d}}$ равна $k \leqslant d-1$. Элемент $\alpha(s) \in \mathcal{S}_{d}$ является циклической перестановкой длины $k$ тогда и только тогда, когда s удовлетворяет следующему условию:

(*) существует слово $w \in W(s)$, граф которого $\Gamma_{w}$ является деревом.

Более того, элемент s, удовлетворяющий условию $(*)$, однозначно определяется ииклической перестановкой $\alpha(s)$.

ДокАзАтельство. Покажем, что если $s$ удовлетворяет условию $(*)$, то существует ровно $k=\ln (s)$ слов $w_{1}, \ldots, w_{k} \in W(s)$ таких, что графы $\Gamma_{w_{i}}$ являются простыми путями, если мы движемся по ребрам в соответствии с их 
нумерацией. Действительно, легко видеть, что из леммы 2.1 следует существование слова $w_{1}=x_{\left(i_{1}, i_{2}\right)} x_{\left(i_{2}, i_{3}\right)} \ldots x_{\left(i_{k-1}, i_{k}\right)}$, граф которого $\Gamma_{w_{1}}$ является простым путем. Покажем, что если мы передвинем букву $x_{\left(i_{k-1}, i_{k}\right)}$ в крайнее положение влево, то мы снова получим определяющее тот же самый элемент $s$ слово $w_{2}$, граф которого $\Gamma_{w_{2}}$ также является простым путем. Действительно, имеем

$$
\begin{aligned}
s & \left.=x_{\left(i_{1}, i_{2}\right)} \cdot \ldots \cdot \frac{x_{\left(i_{k-2}, i_{k-1}\right)} \cdot x_{\left(i_{k-1}, i_{k}\right)}}{x_{\left(i_{k-3}, i_{k-2}\right)} \cdot\left(x_{\left(i_{k-2}, i_{k}\right)}\right.} \cdot x_{\left(i_{k-2}, i_{k-1}\right)}\right)=\ldots \\
& =x_{\left(i_{1}, i_{2}\right)} \cdot \ldots \cdot \frac{\left.x_{\left(i_{k-2}, i_{k-1}\right)}\right)}{}
\end{aligned}
$$

Повторяя такие преобразования $k$ раз, мы найдем искомые слова $w_{1}, \ldots, w_{k}$.

Имеем, что $\alpha(s)=\left(i_{1}, i_{2}\right) \ldots\left(i_{k-2}, i_{k-1}\right)\left(i_{k-1}, i_{k}\right)$ является циклической перестановкой длины $k$. С другой стороны, если $\sigma \in \mathcal{S}_{d}$ - циклическая перестановка длины $k$, то она может быть представлена как произведение $k-1$ транспозиций: $\sigma=\left(i_{1}, i_{2}\right) \ldots\left(i_{k-2}, i_{k-1}\right)\left(i_{k-1}, i_{k}\right)$, и очевидно, что $\alpha(s)=\sigma$ для $s=x_{\left(i_{1}, i_{2}\right)} \cdot \ldots \cdot x_{\left(i_{k-2}, i_{k-1}\right)} \cdot x_{\left(i_{k-1}, i_{k}\right)}$, а граф $\Gamma_{x_{\left(i_{1}, i_{2}\right)} \ldots x_{\left(i_{k-2}, i_{k-1}\right)} x_{\left(i_{k-1}, i_{k}\right)}}$ удовлетворяет условию $(*)$.

Наконец, если мы фиксируем множество $\left\{i_{1}, \ldots, i_{k}\right\} \subset I_{d}$, то имеется ровно $(k-1)$ ! различных циклических перестановок в $\mathcal{S}_{d}$ длины $k$, циклически переставляющих элементы множества $\left\{i_{1}, \ldots, i_{k}\right\}$. С другой стороны, имеется ровно $k$ ! различных простых путей, соединяющих вершины $v_{i_{1}}, \ldots, v_{i_{k}}$. Следовательно, элементы $s$, удовлетворяющие условию $(*)$, однозначно определяются циклическими перестановками $\alpha(s)$.

ЛЕмма 2.7. Пусть элемент $s=x_{\left(i_{1}, i_{2}\right)}^{2} \cdot x_{\left(i_{3}, i_{4}\right)}^{2} \cdot \ldots \cdot x_{\left(i_{2 k-1}, i_{2 k}\right)}^{2}$ является произведением квадратов некоторых образующи полугруппы $S_{T_{d}}$ и таков, что граф $\Gamma_{w}$ слова $w=x_{\left(i_{1}, i_{2}\right)}^{2} x_{\left(i_{3}, i_{4}\right)}^{2} \ldots x_{\left(i_{2 k-1}, i_{2 k}\right)}^{2}$ связен. Тогда

$$
s=\psi_{V\left(\Gamma_{w}\right)}\left(h_{d_{1}, k-d_{1}-1}\right),
$$

где $d_{1}=\left|V\left(\Gamma_{w}\right)\right|-$ число вершин графа $\Gamma_{w} u \psi_{V\left(\Gamma_{w}\right)}\left(h_{d_{1}, k-d_{1}-1}\right)$ - образ элемента Гурвица рода $k-d_{1}-1$ полугруппь $S_{T_{d_{1}}, 1}$ при вложении $\psi_{V\left(\Gamma_{w}\right)}: \Sigma_{d_{1}} \hookrightarrow \Sigma_{d}$, индуиированном вложением $V\left(\Gamma_{w}\right) \hookrightarrow I_{d}$.

ДокАзАтельство. Применив аргументы, аналогичные тем, которые использовались в доказательствах лемм 2.1-2.3, лемма непосредственно следует из условия о том, что граф $\Gamma_{w}$ связен, и из соотношений $(2.5)$ и (2.6).

Лемма 2.8. Для любого $s \in S_{T_{d}}$ разность $\ln (s)-l_{t}(\alpha(s))$ является неотрицательным четным числом и существуют элемент $\widetilde{s} \in S_{T_{d}}$ и элемент $\bar{s}$, представимый в виде произведения квадратов некоторых порождающих полугруппи $S_{T_{d}}$ элементов $x_{(i, j)}$ ( $u$, следовательно, принадлежащий полугрупne $\left.S_{T_{d}, \mathbf{1}}\right)$, maкuе, что:
(i) $s=\widetilde{s} \cdot \bar{s}$;
(ii) $\ln (\widetilde{s})=l_{t}(\alpha(s))$;
(iii) $\alpha(\widetilde{s})=\alpha(s)$.

Более того, элемент $\widetilde{s}$ однозначно определяется условиями (i)-(iii). 
Доказательство. Рассмотрим граф $\Gamma_{w}$ некоторого слова $w \in W(s)=\{w \in$ $W \mid \varphi(w)=s\}$. Он распадается в несвязное объединение своих связных компонент: $\Gamma_{w}=\Gamma_{w, 1} \sqcup \cdots \sqcup \Gamma_{w, l}$. Согласно лемме 2.6 элемент $s$ однозначно (с точностью до перестановки множителей) разлагается в произведение попарно коммутирующих множителей: $s=\varphi\left(w_{1}\left(\overline{x_{(i, j)}}\right)\right) \cdot \ldots \cdot \varphi\left(w_{l}\left(\overline{x_{(i, j)}}\right)\right)$, где $w_{i}\left(\overline{x_{(i, j)}}\right)-$ слово, состоящее из букв $x_{(i, j)}$ таких, что $\Gamma_{w_{i}}=\Gamma_{w, i}$. Пусть $s_{i}=\varphi\left(w_{i}\right) \in S_{T_{d}}-$ элемент, определяемый словом $w_{i}$. Имеем $\left(\mathcal{S}_{d}\right)_{s_{i}} \cap\left(\mathcal{S}_{d}\right)_{s_{j}}=\mathbf{1}$ при $i \neq j$; в частности, $\alpha\left(s_{i}\right)$ и $\alpha\left(s_{j}\right)$ - это коммутирующие перестановки, и множества $V\left(\Gamma_{w, i}\right)$ и $V\left(\Gamma_{w, j}\right)$, на которых каждая из них действует нетривиально, не пересекаются. Поэтому лемму достаточно доказать для элементов $s=\varphi(w)$ со связным графом $\Gamma_{w}$.

Пусть $s=\varphi(w)$ таков, что граф $\Gamma_{w}$ связен. Применив лемму 2.1 , легко видеть, что можно найти такое представление элемента $s$ в виде слова, состоящего из букв $x_{(i, j)}$, что

$$
s=x_{\left(j_{1}, j_{2}\right)} \cdot \ldots \cdot x_{\left(j_{k-1}, j_{k}\right)} \cdot s_{1},
$$

и множество $\left\{v_{j_{1}}, \ldots, v_{j_{k_{i}}}\right\}$ состоит из всех вершин графа $\Gamma_{w}$.

Пусть $x_{\left(j_{a}, j_{b}\right)}$ - первый множитель элемента $s_{1}$, если $s_{1} \neq x_{\mathbf{1}}$. Тогда из соотношений (2.3) и леммы 2.2 следует, что $s$ может быть записан в виде $s=s^{\prime} \cdot x_{\left(j_{a}, j_{b}\right)}^{2}$. Отметим, что $x_{\left(j_{a}, j_{b}\right)}^{2} \in S_{T_{d}, \mathbf{1}}$ и $\ln \left(s^{\prime}\right)=\ln (s)-2<\ln (s)$, т. е. элемент $s$ может быть записан в виде $s=\widetilde{s}_{1} \cdot \bar{s}_{1}$, где $\ln \left(\widetilde{s}_{1}\right)<\ln (s)$ и $\bar{s}_{1} \in S_{T_{d}, \mathbf{1}}$, кроме того, $\alpha\left(\widetilde{s}_{1}\right)=\alpha(s)$, так как $\bar{s}_{1} \in S_{T_{d}, \mathbf{1}}$. Повторяя, если необходимо, вышеприведенные аргументы для элементов $\widetilde{s}_{1}, \ldots$, в результате получим, что $s$ может быть записан в виде $s=\widetilde{s} \cdot \bar{s}$, где $\bar{s} \in S_{T_{d}, \mathbf{1}}-$ произведение квадратов некоторых элементов $x_{(i, j)}$ и $\widetilde{s}=s_{1} \cdot \ldots \cdot s_{m} \in S_{T_{d}}$; здесь при $1 \leqslant i \leqslant m$ элементы $s_{i}=x_{\left(j_{1, i}, j_{2, i}\right)} \cdot \ldots \cdot x_{\left(j_{k_{i}-1, i}, j_{k_{i}, i}\right)}$ таковы, что подмножества $\left\{j_{1, i}, \ldots, j_{k_{i}, i}\right\}$ и $\left\{j_{1, l}, \ldots, j_{k_{l}, l}\right\}$ множества $I_{d}$ имеют пустое пересечение при $i \neq l$. Следовательно,

$$
\alpha(s)=\alpha(\widetilde{s})=\left(j_{k_{1}, 1}, \ldots, j_{1,1}\right) \ldots\left(j_{k_{m}, m}, \ldots, j_{1, m}\right),
$$

и поэтому $\ln (\widetilde{s})=l_{t}(\alpha(s))$.

Согласно предложению 2.1 (с точностью до перестановки) элементы $s_{i}$ определяются однозначно своими произведениями $\alpha\left(s_{i}\right)$. Лемма доказана.

ПреДЛОЖЕНИЕ 2.2. Пусть $s \in S_{T_{d}}$ таков, что $\alpha(s)=\left(i_{1}, i_{2}, \ldots, i_{k}\right)$ является ииклической перестановкой и множество вериин $V(s)$ графа $\Gamma_{w}$, $w \in W(s)$, совпадает с множеством $\left\{i_{1}, i_{2}, \ldots, i_{k}\right\} \subset I_{d}$. Тогда

$$
s=x_{\left(i_{1}, i_{2}\right)} \cdot x_{\left(i_{2}, i_{3}\right)} \cdot \ldots \cdot x_{\left(i_{k-1}, i_{k}\right)} \cdot x_{\left(i_{2}, i_{1}\right)}^{2 n},
$$

где $2 n=\ln (s)-k+1$.

\section{ДоКАЗАТЕЛЬСТво следует из леммы 2.8 и леммы 2.5 .}

ПреДЛОЖЕНИЕ 2.3. Пустъ элемент $s=\varphi(w) \in S_{T_{d}}$ таков, что $\Gamma_{w}$ является связным графом, и если $\alpha(s)=\prod_{j=1}^{m}\left(i_{1, j}, i_{2, j}, \ldots, i_{k_{j}, j}\right)$ - разложение в произведение циклических перестановок, то либо $m>1$, либо $V\left(\Gamma_{w}\right) \neq$ $\left\{i_{1,1}, i_{2,1}, \ldots, i_{k_{1}, 1}\right\}$ в случае $m=1$. Обозначим

$$
J=\left\{i_{1,1}, \ldots i_{1, m}\right\} \cup\left(V\left(\Gamma_{w}\right) \backslash \bigcup_{j=1}^{m}\left\{i_{1, j}, i_{2, j}, \ldots, i_{k_{j}, j}\right\}\right) \subset I_{d} .
$$


Тогда

$$
s=\psi_{J}\left(h_{d_{1}, g}\right) \cdot \prod_{j=1}^{m}\left(x_{\left(i_{1, j}, i_{2, j}\right)} \cdot x_{\left(i_{2, j}, i_{3, j}\right)} \cdot \ldots \cdot x_{\left(i_{k_{j}-1, j}, i_{k_{j}, j}\right)}\right)
$$

где $h_{d_{1}, g} \in \Sigma_{T_{d_{1}}}$ - элемент Гурвица рода $g, d_{1}=|J|, g=\frac{\ln (s)-d_{1}+1}{2}$ и вложение $\psi_{J}: \Sigma_{d_{1}} \hookrightarrow \Sigma_{d}$ индуцировано вложением $J \rightarrow I_{d}$.

ДоказАтельство. Согласно лемме 2.8 элемент $s$ может быть записан в виде

$$
s=\widetilde{s} \cdot \prod_{j=1}^{m}\left(x_{\left(i_{1, j}, i_{2, j}\right)} \cdot x_{\left(i_{2, j}, i_{3, j}\right)} \cdot \ldots \cdot x_{\left(i_{k_{j}-1, j}, i_{k_{j}, j}\right)}\right),
$$

где $\widetilde{s}$ - произведение квадратов некоторых порождающих элементов $x_{(a, b)}$ полугруппы $S_{T_{d}}$, причем из условий предложения следует, что $\ln (\widetilde{s}) \neq 0$. Рассмотрим один из множителей $x_{(a, b)}^{2}$, входящих в разложение элемента $\widetilde{s}$ на множители. Если $a$ (либо $b$ ) принадлежит некоторому множеству $\left\{i_{1, j}, \ldots, i_{k_{j}, j}\right\}$, то согласно леммам 2.4, 2.5 этот множитель может быть заменен в произведении $(2.7)$ на $x_{\left(i_{1, j}, b\right)}^{2}$ и эта замена не меняет элемент $s$. Поэтому мы можем считать, что для каждого множителя $x_{(a, b)}^{2}$, входящего в разложение элемента $\widetilde{s}$ на множители, имеют место следующие четыре возможности:

1) $\{a, b\} \subset V\left(\Gamma_{w}\right) \backslash \bigcup_{j=1}^{m}\left\{i_{1, j}, i_{2, j}, \ldots, i_{k_{j}, j}\right\}$;

2) $a=i_{1, j}$ для некоторого $j \in[1, m]$, a $b \in V\left(\Gamma_{w}\right) \backslash \bigcup_{j=1}^{m}\left\{i_{1, j}, i_{2, j}, \ldots, i_{k_{j}, j}\right\}$;

3) $\{a, b\}=\left\{i_{1, j_{1}}, i_{1, j_{2}}\right\}$ для некоторых $j_{1}, j_{2} \in[1, m]$;

4) $\{a, b\}=\left\{i_{1, j}, i_{2, j}\right\}$ для некоторого $j \in[1, m]$.

Обозначим через $\widetilde{w}$ слово, представляющее описанное выше разложение на множители элемента $\widetilde{s}$. Из связности графа $\Gamma_{w}$ следует, что граф $\Gamma_{\widetilde{w}}$ также связен, $J \subset V\left(\Gamma_{\widetilde{w}}\right)$ и, более того, для каждого $j \in[1, m]$ найдется $b \notin\left\{i_{1, j}, \ldots, i_{k_{j}, j}\right\}$ такое, что $x_{\left(i_{1, j}, b\right)}^{2}$ является подсловом слова $\widetilde{w}$. Если в разложение $\widetilde{w}$ входит подслово $x_{\left(i_{1, j}, i_{2, j}\right)}^{2}$ для некоторого $j$, то согласно лемме 2.3 имеем (напомним, что элементы $x_{(a, b)}^{2}$ принадлежат центру полугруппы $\left.S_{T_{d}}\right)$

$$
\begin{aligned}
& \underline{x_{\left(i_{1, j}, i_{2, j}\right)}^{2} \cdot x_{\left(i_{1, j}, b\right)}^{2}} \cdot x_{\left(i_{1, j}, i_{2, j}\right)}=\left(x_{\left(i_{1, j}, b\right)}^{2} \cdot \underline{\left.x_{\left(i_{2, j}, b\right)}^{2}\right) \cdot x_{\left(i_{1, j}, i_{2, j}\right)}}\right. \\
& =x_{\left(i_{1, j}, b\right)}^{2} \cdot\left(x_{\left(i_{1, j}, b\right)}^{2} \cdot x_{\left(i_{1, j}, i_{2, j}\right)}\right),
\end{aligned}
$$

поэтому мы можем считать, что $V\left(\Gamma_{\widetilde{w}}\right)=J$ и граф $\Gamma_{\widetilde{w}}$ связен. Чтобы завершить доказательство предложения, достаточно применить лемму 2.7.

Из предложений 2.2, 2.3 следует

Теорема 2.1 [7], [8]. Элементы $s_{1}, s_{2} \in S_{T_{d}}^{\mathcal{S}_{d}}$ равны тогда и только тогда, когда $\alpha\left(s_{1}\right)=\alpha\left(s_{2}\right) u \ln \left(s_{1}\right)=\ln \left(s_{2}\right)$.

ПрЕДЛОЖЕНИЕ 2.4. Если $s \in S_{T_{d}}^{\mathcal{S}_{d}} u \ln (s) \geqslant l_{t}(\alpha(s))+2(d-1)$, то существует разложение $s=\widetilde{s} \cdot \bar{s}$, где $\widetilde{s}=h_{d, g}, g=\frac{1}{2}\left(\ln (s)-l_{t}(\alpha(s))\right)-d+1, u \bar{s}-$ такой элемент, что $\ln (\bar{s})=l_{t}(\alpha(s)), \alpha(\bar{s})=\alpha(s)$ и элемент $\bar{s}$ определен однозначно своим произведением $\alpha(s)$. 
ДокАЗАТЕЛЬСтво. Согласно предложениям 2.2, 2.3 элемент $s$ может быть разложен в произведение: $s=\widetilde{s} \cdot \bar{s} \in S_{T_{d}}$, где $\widetilde{s} \in S_{T_{d}}^{\mathcal{S}_{d}}-$ произведение квадратов некоторых элементов $x_{(i, j)}$ и $\bar{s}$ - такой элемент, что

$$
\alpha(s)=\alpha(\bar{s})=\left(j_{1,1}, \ldots, j_{k_{1}, 1}\right) \ldots\left(j_{1, m}, \ldots, j_{k_{m}, m}\right)
$$

и $\ln (\bar{s})=l_{t}(\alpha(s))$. Заметим, что $\ln (\widetilde{s}) \geqslant 2(d-1)$, так как $\ln (\bar{s})=l_{t}(\alpha(s))$ и $\ln (s) \geqslant l_{t}(\alpha(s))+2(d-1)$.

Рассмотрим графы $\Gamma_{\widetilde{w}}, \Gamma_{\bar{w}}$ и $\Gamma_{\widetilde{w}}$, где $\widetilde{w} \in W(\widetilde{s}), \bar{w} \in W(\bar{s})$ и $\widetilde{w} \bar{w} \in W(s)$. Покажем, что существует разложение на множители $s=\widetilde{s} \cdot \bar{s}$ такое, что $V_{\widetilde{s}}=I_{d}$ и граф $\Gamma_{\widetilde{w}}$ связен. Во-первых, имеем $V_{s}=I_{d}$, так как $\left(\mathcal{S}_{d}\right)_{s}=\mathcal{S}_{d}$. Предположим, что либо $V_{\widetilde{s}} \neq I_{d}$, либо граф $\Gamma_{\widetilde{w}}$ не связен для некоторого разложения $s=\widetilde{s} \cdot \bar{s}$, и пусть $\widetilde{s}=\varphi\left(\widetilde{w}\left(\overline{x_{(i, j)}^{2}}\right)\right)$ и $\bar{s}=\varphi\left(\bar{w}\left(\overline{x_{(i, j)}}\right)\right)$. Поскольку $\ln (\widetilde{s}) \geqslant 2(d-1)$, из леммы 2.3 следует, что существует связная компонента $\Gamma_{1}$ графа $\Gamma_{\widetilde{w}}$ такая, что для любой пары вершин $v_{i_{1}}, v_{i_{2}} \in \Gamma_{1}$ мы можем найти слово $\widetilde{w} \in W(\widetilde{s})$ такое, что $\widetilde{s}=\left(x_{\left(i_{1}, i_{2}\right)}^{2}\right)^{2} \cdot \widetilde{s}^{\prime}$. Затем, так как $V_{s}=I_{d}$, найдется пара вершин $v_{i_{0}}, v_{i_{2}} \in V_{\bar{s}}$ такая, что $v_{i_{0}} \notin V_{\widetilde{s}}, v_{i_{2}} \in V_{\widetilde{s}}$ и $\bar{s}=\bar{s}^{\prime} \cdot x_{\left(i_{0}, i_{2}\right)}$. Согласно лемме 2.3 имеем

$s=\widetilde{s} \cdot \bar{s}=\bar{s}^{\prime} \cdot x_{\left(i_{0}, i_{2}\right)} \cdot x_{\left(i_{1}, i_{2}\right)}^{2} \cdot x_{\left(i_{1}, i_{2}\right)}^{2} \cdot \bar{s}^{\prime}=\bar{s}^{\prime} \cdot x_{\left(i_{0}, i_{2}\right)} \cdot x_{\left(i_{0}, i_{1}\right)}^{2} \cdot x_{\left(i_{1}, i_{2}\right)}^{2} \cdot \widetilde{s}^{\prime}=\widetilde{s} \cdot \widetilde{s}_{1}$,

где для слова $\widetilde{w}_{1} \in W\left(\widetilde{s}_{1}\right)$ либо $V_{\widetilde{s}_{1}}=V_{\widetilde{s}} \cup\left\{i_{0}\right\}$ и граф $\Gamma_{\widetilde{w}_{1}}$ имеет то же самое число связных компонент, что и граф $\Gamma_{\widetilde{w}}$, а число вершин одной из его связных компонент увеличилось на единицу, либо число связных компонент графа $\Gamma_{\widetilde{w}_{1}}$ строго меньше числа связных компонент графа $\Gamma_{\widetilde{w}}$. Повторив эти преобразования несколько раз, в результате получим разложение $s=\widetilde{s} \cdot \bar{s}$ такое, что $V_{\widetilde{s}}=I_{d}$ и граф $\Gamma_{\widetilde{w}_{1}}$ связен. Теперь, чтобы завершить доказательство предложения, достаточно еще раз применить лемму 2.3 .

ПредлОЖенИЕ 2.5. Существует (и притом единственный) гомоморфизм $r: \Sigma_{d} \rightarrow S_{T_{d}}$ mакой, что:

(i) $\alpha\left(r\left(x_{\sigma}\right)\right)=\sigma \partial л я \sigma \in \mathcal{S}_{d}$;

(ii) $\ln \left(r\left(x_{\sigma}\right)\right)=l_{t}(\sigma)$;

(iii) $r_{\mid S_{T_{d}}}=\mathrm{Id}$.

ДокАзательство. Каждая перестановка $\sigma \in \mathcal{S}_{d}, \sigma \neq 1$, может быть разложена в произведение попарно коммутирующих циклических перестановок: $\sigma=\sigma_{1} \ldots \sigma_{m}$, и такое разложение единственно с точностью до перестановки множителей. Согласно предложению 2.1 каждая из циклических перестановок $\sigma_{i}$ однозначно определяет элемент $s_{i} \in S_{T_{d}}$ такой, что $\ln \left(s_{i}\right)=k_{i}-1$ и $\alpha\left(s_{i}\right)=\sigma_{i}$, где $k_{i}$ - длина циклической перестановки $\sigma_{i}$, и, следовательно, произведение $s(\sigma)=s_{1} \cdot \ldots \cdot s_{m} \in S_{T_{d}}$ однозначно определяется по $\sigma$. Легко видеть, что отображение $\sigma \mapsto s(\sigma)$ определяет гомоморфизм $r: \Sigma_{d} \rightarrow S_{T_{d}}$, заданный формулой $r\left(x_{\sigma}\right)=s(\sigma)$ на множестве порождающих элементов полугруппы $\Sigma_{d}$. Очевидно, что $\ln _{t}(s)=\ln (r(s))$ и $r_{\mid S_{T_{d}}}=\mathrm{Id}$.

Гомоморфизм $r: \Sigma_{d} \rightarrow S_{T_{d}}$, определенный в предложении 2.5 , называется восстанавливающим гомоморфизмом, а число $\ln _{t}(s)=\ln (r(s))$ называется транспозиционной длиной элемента $s \in \Sigma_{d}$. 
2.3. Разложения единицы в произведения транспозиций. Рассмотрим полугруппу $S_{T_{d}, \mathbf{1}}$.

Теорема 2.2. Полугруппа $S_{T_{d}, 1}$ является коммутативной и порождается элементами $s_{(i, j)}=x_{(i, j)}^{2},\{i, j\} \subset I_{d}$, связанными соотношениями

$$
s_{\left(i_{1}, i_{2}\right)} \cdot s_{\left(i_{2}, i_{3}\right)}=s_{\left(i_{1}, i_{2}\right)} \cdot s_{\left(i_{1}, i_{3}\right)}=s_{\left(i_{2}, i_{3}\right)} \cdot s_{\left(i_{1}, i_{3}\right)}
$$

для упорядоченных троек $\left\{i_{1}, i_{2}, i_{3}\right\}_{\text {ord }} \subset I_{d} u$

$$
s_{\left(i_{1}, i_{2}\right)} \cdot s_{\left(i_{3}, i_{4}\right)}=s_{\left(i_{3}, i_{4}\right)} \cdot s_{\left(i_{1}, i_{2}\right)}
$$

для упорядоченных четверок $\left\{i_{1}, i_{2}, i_{3}, i_{4}\right\}_{\text {ord }} \subset I_{d}$. Более того, любой элемент $s \in S_{T_{d}, \mathbf{1}}$ имеет нормальную форму, т.е. он может быть единственным образом записан в виде

$$
\begin{aligned}
s=\left(s_{\left(i_{1,1}, i_{2,1}\right)}^{k_{1}} \cdot s_{\left(i_{2,1}, i_{3,1}\right)} \cdot \ldots \cdot s_{\left(i_{j_{1}-1,1}, i_{j_{1}, 1}\right)}\right) \cdot \ldots \\
\ldots \cdot\left(s_{\left(i_{1, n}, i_{2, n}\right)}^{k_{n}} \cdot s_{\left(i_{2, n}, i_{3, n}\right)} \cdot \ldots \cdot s_{\left(i_{j_{n}-1, n}, i_{j_{n}, n}\right)}\right),
\end{aligned}
$$

где $1 \leqslant i_{1,1}<i_{1,2}<\cdots<i_{1, n} \leqslant d-1, k_{l} \in \mathbb{N}$ для $l=1, \ldots, n$, множества $M_{l}=$ $\left\{i_{1, l}<i_{2, l}<\cdots<i_{j_{l}, l}\right\}, 1 \leqslant l \leqslant n$, являттся подмножествами множества $I_{d}$, состоящими из $j_{l} \geqslant 2$ элементов таких, что $M_{l_{1}} \cap M_{l_{2}}=\varnothing$ при $l_{1} \neq l_{2}$.

ДокАЗАТЕЛьство. Из леммы 2.8 следует, что полугруппа $S_{T_{d}, \mathbf{1}}$ порождается элементами $s_{(i, j)}$. Согласно лемме 2.3 для элементов $s_{(i, j)}$ выполнены соотношения (2.8) и (2.9).

Как и в доказательстве предложения 2.4 , с каждым элементом $s=s_{\left(j_{1}, j_{2}\right)} \cdot \ldots$ $\ldots \cdot s_{\left(j_{m-1}, j_{m}\right)}$ мы можем связать граф $\Gamma_{w}$, где $w$ - слово, состоящее из букв $s_{(i, j)}$ и представляющее элемент $s$. Граф $\Gamma_{w}$ распадается в несвязное объединение своих связных компонент: $\Gamma_{w}=\Gamma_{w, 1} \sqcup \cdots \sqcup \Gamma_{w, n}$. Из соотношений (2.3) легко следует, что $w=w_{1}\left(\overline{s_{(i, j)}}\right) \ldots w_{n}\left(\overline{s_{(i, j)}}\right)$, где слово $w_{l}\left(\overline{s_{(i, j)}}\right)$, состоящее из букв $s_{(i, j)}$, является таким, что $\Gamma_{w_{l}}=\Gamma_{w, l}$. Пусть $s_{l} \in S_{T_{d}, \mathbf{1}}-$ элемент, определяемый словом $w_{l}$, т. е. $s_{l}=\varphi\left(w_{l}\right)$.

Из соотношений (2.8) и (2.9) следует, что каждый элемент $s_{l}$ может быть записан в виде

$$
s_{l}=s_{\left(i_{1, l}, i_{2, l}\right)}^{k_{l}} \cdot s_{\left(i_{2, l}, i_{3, l}\right)} \cdot \ldots \cdot s_{\left(i_{j_{l}-1, l}, i_{j_{l}, l}\right)},
$$

где элементы множества $M_{l}=\left\{i_{1, l}<i_{2, l}<\cdots<i_{j_{l}, l}\right\}, 1 \leqslant l \leqslant n$, находятся во взаимно однозначном соответствии с вершинами связной компоненты $\Gamma_{w, l}$ графа $\Gamma_{w}$.

ЗАмечАнИЕ 2.1. Элемент $s_{\left(i_{1, l}, i_{2, l}\right)}^{k_{l}} \cdot s_{\left(i_{2, l}, i_{3, l}\right)} \cdot \ldots \cdot s_{\left(i_{j_{l}-1, l}, i_{j_{l}, l}\right)}$ в $(2.10)$ является элементом Гурвица $h_{j_{l}, k_{l}-1}$ полугруппы $S_{T_{j_{l}}, 1}$, если мы рассматриваем $S_{T_{j_{l}}, 1}$ как подполугруппу полугруппы $S_{T_{d}, \mathbf{1}}$ и вложение определено естественным вложением $M_{l} \hookrightarrow I_{d}$.

ПредложениЕ 2.6. Элемент Гурвица $h_{d, g}$ принадлежит иентру полугруппы $\Sigma_{d}$, и он неподвижен при действии сопряжениями группы $\mathcal{S}_{m}$ на полугруппе $\Sigma_{d}$. Для $h_{d, g_{1}}, h_{d, g_{2}}$ имеем

$$
h_{d, g_{1}} \cdot h_{d, g_{2}}=h_{d, g_{1}+g_{2}+d-1} .
$$


ДокАЗАТЕльство. Первая часть предложения следует из предложения 1.1, так как, с одной стороны, $\alpha\left(h_{d, g}\right)=\mathbf{1}$ и транспозиции $(i, i+1), i=1, \ldots, d-1$, порождают группу $\left(\mathcal{S}_{d}\right)_{h_{d, g}}$, а с другой стороны, они порождают всю группу $\mathcal{S}_{d}$. Вторая часть предложения следует из предложения 2.4.

Более того, в качестве следствия из теорем 2.4, 2.2 мы получаем, что элемент Гурвица $h_{d, g}$ определяется однозначно в полугруппе $S_{T_{d}}$ своей длиной и следующими двумя условиями.

СледствиЕ 2.1 (теорема Клебша-Гурвица [1]). Пусть элемент $s \in S_{T_{d}}$ удовлетворлет следующим условиям:

(i) $\left(\mathcal{S}_{d}\right)_{s}=\mathcal{S}_{d}$;

(ii) $\alpha(s)=1$.

Тогда $\ln (s) \geqslant 2(d-1)$ u $s=h_{d, g}$, где $g=\frac{\ln (s)}{2}-d+1$.

2.4. Разложения на множители в симметрических группах (общий случай). В этом пункте будет доказано следующее обобщение предложения 2.4 .

Теорема 2.3. Пусть $s=x_{\sigma_{1}} \cdot \ldots \cdot x_{\sigma_{m}} \cdot \bar{s} \in \Sigma_{d}$, где $\bar{s} \in S_{T_{d}}$. Для $j=1, \ldots, m$ обозначим через $\sigma_{j, 0}$ канонический представитель типа $t\left(\sigma_{j}\right)$ (см. определения в п. 2.1), и пусть

$$
\sigma=\sigma(s)=\left(\sigma_{1,0} \ldots \sigma_{m, 0}\right)^{-1} \alpha(s) .
$$

Ecлu $s \in \Sigma_{d}^{\mathcal{S}_{d}} u \ln (\bar{s})=k \geqslant 3(d-1)$, mo

$$
s=x_{\sigma_{1,0}} \cdot \ldots \cdot x_{\sigma_{m, 0}} \cdot r\left(x_{\sigma}\right) \cdot h_{d, g},
$$

где $g=\frac{k-\ln _{t}\left(x_{\sigma}\right)}{2}-d+1$.

ДокАзАтЕЛЬство. Покажем, что существует разложение

$$
s=x_{\sigma_{1}^{\prime}} \cdot \ldots \cdot x_{\sigma_{m}^{\prime}} \cdot x_{\left(i_{1}, j_{1}\right)} \cdot \ldots \cdot x_{\left(i_{k}, j_{k}\right)}=x_{\sigma_{1}^{\prime}} \cdot \ldots \cdot x_{\sigma_{m}^{\prime}} \cdot \bar{s}_{1}
$$

такое, что $t\left(\sigma_{i}\right)=t\left(\sigma_{i}^{\prime}\right)$ для $i=1, \ldots, m$, граф $\Gamma_{\bar{w}_{1}}$, ассоциированный со словом $\bar{w}_{1}=x_{\left(i_{1}, j_{1}\right)} \ldots x_{\left(i_{k}, j_{k}\right)} \in W\left(\bar{s}_{1}\right)$, связен и множество $V_{\bar{s}_{1}}$ его вершин совпадает с множеством $I_{d}$.

Действительно, пусть $w \in W(\bar{s})$, предположим, что либо $V_{\bar{s}} \neq I_{d}$, либо граф $\Gamma_{\bar{w}}$ не связен. Поскольку $\ln (\bar{s}) \geqslant 3(d-1)$, найдется связная компонента $\Gamma_{1}$ графа $\Gamma_{w}$ такая, что число ее ребер больше числа ее вершин. Тогда из доказательства предложения 2.4 следует, что для любых двух вершин $v_{i_{1}}, v_{i_{2}}$, принадлежащих множеству вершин $V\left(\Gamma_{1}\right)$ графа $\Gamma_{1}$, найдется слово $w^{\prime} \in W$ такое, что $\bar{s}=x_{\left(i_{1}, i_{2}\right)}^{2} \cdot \varphi\left(w^{\prime}\right)$ и вершины из $V\left(\Gamma_{1}\right)$ принадлежат одной и той же связной компоненте графа $\Gamma_{x_{i_{1}, i_{2}}^{2} w^{\prime}}$ З Затем, так как $\left(\mathcal{S}_{d}\right)_{s}=\mathcal{S}_{d}$, для некоторого $l, 1 \leqslant l \leqslant m$, найдется перестановка $\sigma_{l}$ такая, что $\sigma_{l}\left(i_{1}, i_{2}\right) \sigma_{l}^{-1}=\left(i_{0}, j_{0}\right)$, где или вершина $v_{i_{0}}$, или вершина $v_{j_{0}}$ (но не обе сразу) не принадлежит множеству $V\left(\Gamma_{1}\right)$. Не ограничивая общности, мы можем предполагать, что $l=m$. 
Имеем

$$
\begin{aligned}
s & =x_{\sigma_{1}} \cdot \ldots \cdot x_{\sigma_{m}} \cdot \bar{s}=x_{\sigma_{1}} \cdot \ldots \cdot x_{\sigma_{m}} \cdot x_{\left(i_{1}, i_{2}\right)}^{2} \cdot \varphi\left(w^{\prime}\right) \\
& =x_{\sigma_{1}} \cdot \ldots \cdot x_{\sigma_{m-1}} \cdot x_{\left(i_{0}, j_{0}\right)} \cdot x_{\sigma_{m}} \cdot x_{\left(i_{1}, i_{2}\right)} \cdot \varphi\left(w^{\prime}\right) \\
& =x_{\sigma_{1}} \cdot \ldots \cdot x_{\sigma_{m-1}} \cdot \rho\left(\left(i_{0}, j_{0}\right)\right)\left(x_{\sigma_{m}}\right) \cdot x_{\left(i_{0}, j_{0}\right)} \cdot x_{\left(i_{1}, i_{2}\right)} \cdot \varphi\left(w^{\prime}\right) \\
& =x_{\sigma_{1}} \cdot \ldots \cdot x_{\sigma_{m-1}} \cdot \rho\left(\left(i_{0}, j_{0}\right)\right)\left(x_{\sigma_{m}}\right) \cdot \varphi\left(w^{\prime \prime}\right),
\end{aligned}
$$

где $w^{\prime \prime}=x_{\left(i_{0}, j_{0}\right)} x_{\left(i_{1}, j_{1}\right)} w^{\prime}-$ такое слово, что либо множество вершин графа $\Gamma_{w^{\prime \prime}}$ строго содержит множество $V_{\bar{s}}$, либо число связных компонент графа $\Gamma_{w^{\prime \prime}}$ строго меньше числа связных компонент графа $\Gamma_{w^{\prime}}$.

Повторив аналогичные преобразования несколько раз, в результате получим такое разложение элемента $s$ в виде

$$
s=x_{\sigma_{1}^{\prime}} \cdot \ldots \cdot x_{\sigma_{m}^{\prime}} \cdot \bar{s}_{1},
$$

что $\bar{s}_{1} \in S_{T_{d}}, V_{\bar{s}_{1}}=I_{d}$, граф $\Gamma_{\bar{w}_{1}}$ связен и $t\left(\sigma_{j}^{\prime}\right)=t\left(\sigma_{j}\right)$ для $j=1, \ldots, m$. Для этого разложения имеем $\left(\mathcal{S}_{d}\right)_{\bar{s}_{1}}=\mathcal{S}_{d}$ и $\ln \left(\bar{s}_{1}\right) \geqslant 3(d-1)$.

Чтобы завершить доказательство теоремы, используем индукцию по $\mathrm{m}$. Если $m=0$ (т. е. если $s \in S_{T_{d}}$ ), то теорема следует из предложения 2.4.

Пусть $m=1$. Согласно предложению 2.4 имеем $\bar{s}_{1}=h_{d, 0} \cdot \bar{s}^{\prime}$ для некоторого элемента $\bar{s}^{\prime} \in S_{T_{d}}$.

Лемма 2.9. Пусть $\left\{i_{1,1}, \ldots, i_{k_{1}, 1}\right\} \sqcup \cdots \sqcup\left\{i_{1, n}, \ldots, i_{k_{n}, n}\right\}-$ несвязное объединение упорядоченных подмножеств множества $I_{d}$. Тогда элемент Гурвиu, $h_{d, 0}$ может быть представлен как произведение:

$$
h_{d, 0}=\left(x_{\left(i_{1,1}, i_{2,1}\right)} \cdot \ldots \cdot x_{\left(i_{k_{1}-1,1}, i_{k_{1}, 1}\right)}\right) \cdot \ldots \cdot\left(x_{\left(i_{1, n}, i_{2, n}\right)} \cdot \ldots \cdot x_{\left(i_{k_{n}-1, n}, i_{k_{n}, n}\right)}\right) \cdot \bar{h}
$$

где $\bar{h}$ - некоторый элемент полугруппь $S_{T_{d}}^{\mathcal{S}_{d}}$.

ДокАзАтельство. Полугруппа $S_{T_{d, 1}}$ является коммутативной, и элемент Гурвица $h_{d, 0}$ инвариантен при действии сопряжениями группы $\mathcal{S}_{d}$. Следовательно, $h_{d, 0}$ может быть записан в виде

$$
h_{d, 0}=\left(s_{\left(i_{1,1}, i_{2,1}\right)} \cdot \ldots \cdot s_{\left(i_{k_{1}-1,1}, i_{k_{1}, 1}\right)}\right) \cdot \ldots \cdot\left(s_{\left(i_{1, n}, i_{2, n}\right)} \cdot \ldots \cdot s_{\left(i_{k_{n}-1, n}, i_{k_{n}, n}\right)}\right) \cdot \widetilde{h},
$$

где $\widetilde{h}$ - некоторый элемент из $S_{T_{d, \mathbf{1}}}$. Имеем

$$
\begin{aligned}
s_{\left(i_{1, j}, i_{2, j}\right)} & \ldots \cdot s_{\left(i_{k_{j}-1, j}, i_{k_{j}, j}\right)}=x_{\left(i_{1, j}, i_{2, j}\right)}^{2} \cdot \ldots \cdot x_{\left(i_{k_{j}-1, j}, i_{k_{j}, j}\right)}^{2} \\
& =x_{\left(i_{1, j}, i_{2, j}\right)} \cdot\left(x_{\left(i_{2, j}, i_{3, j}\right)}^{2} \cdot \ldots \cdot x_{\left(i_{k_{j}-1, j}, i_{k_{j}, j}\right)}^{2}\right) \cdot x_{\left(i_{1, j}, i_{2, j}\right)}=\ldots \\
& \ldots=\left(x_{\left(i_{1, j}, i_{2, j}\right)} \cdot \ldots \cdot x_{\left(i_{k_{j}-1, j}, i_{k_{j}, j}\right)}\right) \cdot\left(x_{\left(i_{k_{j}-1, j}, i_{k_{j}, j}\right)} \cdot \ldots \cdot x_{\left(i_{1, j}, i_{2, j}\right)}\right),
\end{aligned}
$$

и элементы $x_{\left(i_{l_{1}, j_{1}}, i_{l_{1}+1, j_{1}}\right)}$ и $x_{\left(i_{l_{2}, j_{2}}, i_{l_{2}+1, j_{2}}\right)}$ коммутируют, если $j_{1} \neq j_{2}$. Теперь, чтобы завершить доказательство леммы, заметим, что $\left(\mathcal{S}_{d}\right)_{s_{j}}=\left(\mathcal{S}_{d}\right)_{\bar{s}_{j}}$, где $s_{j}=$ $s_{\left(i_{1, j}, i_{2, j}\right)} \cdot \ldots \cdot s_{\left(i_{k_{j}-1, j}, i_{k_{j}, j}\right)}$ и $\bar{s}_{j}=x_{\left(i_{k_{j}-1, j}, i_{k_{j}, j}\right)} \cdot \ldots \cdot x_{\left(i_{1, j}, i_{2, j}\right)}$. Следовательно, $\bar{h}=\left(\prod \bar{s}_{i}\right) \cdot \widetilde{h} \in S_{T_{d}}^{\mathcal{S}_{d}}$, так как $h_{d, 0} \in S_{T_{d}}^{\mathcal{S}_{d}}$. Лемма доказана. 
Для канонического представителя $\sigma_{m, 0}$ типа $t\left(\sigma_{m}\right)$ существует элемент $\bar{\sigma}_{m} \in \mathcal{S}_{d}$ такой, что $\sigma_{m, 0}=\bar{\sigma}_{m}^{-1} \sigma_{m}^{\prime} \bar{\sigma}_{m}$. Перестановка $\bar{\sigma}_{m}$ может быть разложена в произведение циклических перестановок и каждая из циклических перестановок может быть разложена в произведение транспозиций:

$$
\bar{\sigma}_{m}=\left(\left(i_{1,1}, i_{2,1}\right) \ldots\left(i_{k_{1}-1,1}, i_{k_{1}, 1}\right)\right) \ldots\left(\left(i_{1, n}, i_{2, n}\right) \ldots\left(i_{k_{n}-1, n}, i_{k_{n}, n}\right)\right) .
$$

Рассмотрим элемент

$r\left(x_{\bar{\sigma}_{m}}\right)=\left(x_{\left(i_{1,1}, i_{2,1}\right)} \cdot \ldots \cdot x_{\left(i_{k_{1}-1,1}, i_{k_{1}, 1}\right)}\right) \cdot \ldots \cdot\left(x_{\left(i_{1, n}, i_{2, n}\right)} \cdot \ldots \cdot x_{\left(i_{k_{n}-1, n}, i_{k_{n}, n}\right)}\right) \in S_{T_{d}}$,

где $r$ - восстанавливающий гомоморфизм. Согласно лемме 2.9

$$
h_{d, 0}=r\left(x_{\bar{\sigma}_{m}}\right) \cdot \bar{h}_{m},
$$

где $\bar{h}_{m}$ - некоторый такой элемент, что $\left(\mathcal{S}_{d}\right)_{\bar{h}_{m}}=\mathcal{S}_{d}$. Имеем

$$
\begin{aligned}
s & =x_{\sigma_{m}^{\prime}} \cdot h_{d, 0} \cdot \bar{s}^{\prime}=x_{\sigma_{m}^{\prime}} \cdot r\left(x_{\bar{\sigma}_{m}}\right) \cdot \bar{h}_{m} \cdot \bar{s}^{\prime} \\
& =r\left(x_{\bar{\sigma}_{m}}\right) \cdot x_{\sigma_{m, 0}} \cdot \bar{h}_{m} \cdot \bar{s}^{\prime}=x_{\sigma_{m, 0}} \cdot r\left(x_{\bar{\sigma}_{m}^{\prime}}\right) \cdot \bar{h}_{m} \cdot \bar{s}^{\prime},
\end{aligned}
$$

где $x_{\bar{\sigma}_{m}^{\prime}}=\lambda\left(\sigma_{m, 0}\right)\left(x_{\bar{\sigma}_{m}}\right)$. Имеем также $\bar{s}_{1}^{\prime}=r\left(x_{\bar{\sigma}_{m}^{\prime}}\right) \cdot \bar{h}_{m} \cdot \bar{s}^{\prime} \in S_{T_{d}}$ такой, что $\ln \left(\bar{s}_{1}^{\prime}\right)=k$, кроме того, $\alpha\left(\bar{s}_{1}^{\prime}\right)=\sigma_{m, 0}^{-1} \alpha(s)$ и $\left(\mathcal{S}_{d}\right)_{\bar{s}_{1}^{\prime}}=\mathcal{S}_{d}$. Следовательно, согласно теореме 2.4 выполнено равенство $\bar{s}_{1}^{\prime}=r\left(x_{\sigma}\right) \cdot h_{d, g}$, где $\sigma=\alpha\left(\bar{s}_{1}^{\prime}\right)=\sigma_{m, 0}^{-1} \alpha(s)$ и $g=\frac{k-\ln _{t}\left(x_{\sigma}\right)}{2}-d+1$.

Теперь предположим, что теорема доказана для всех $m<m_{0}$, и рассмотрим элемент

$$
s=x_{\sigma_{1}} \cdot \ldots \cdot x_{\sigma_{m_{0}}} \cdot \bar{s}_{1},
$$

где элемент $\bar{s}_{1} \in S_{T_{d}}$ имеет длину $k \geqslant 3(d-1)$ и является таким, что $\left(\mathcal{S}_{d}\right)_{\bar{s}_{1}}=\mathcal{S}_{d}$. Имеем

$$
\begin{aligned}
s & =x_{\sigma_{1}} \cdot \ldots \cdot x_{\sigma_{m_{0}}} \cdot \bar{s}_{1}=x_{\sigma_{2}^{\prime}} \cdot \ldots \cdot x_{\sigma_{m_{0}}^{\prime}} \cdot x_{\sigma_{1}} \cdot \bar{s}_{1} \\
& =x_{\sigma_{2}^{\prime}} \cdot \ldots \cdot x_{\sigma_{m_{0}}^{\prime}} \cdot x_{\sigma_{1,0}} \cdot \bar{s}_{1}^{\prime}=x_{\sigma_{1,0}} \cdot x_{\sigma_{2}^{\prime \prime}} \cdot \ldots \cdot x_{\sigma_{m_{0}}^{\prime \prime}} \cdot \bar{s}_{1}^{\prime},
\end{aligned}
$$

где $\sigma_{j}^{\prime}=\sigma_{1} \sigma_{j} \sigma_{1}^{-1}$ и $\sigma_{j}^{\prime \prime}=\sigma_{1,0}^{-1} \sigma_{j}^{\prime} \sigma_{1,0}$ для $j=2, \ldots, m$ и элемент $\bar{s}_{1}^{\prime} \in S_{d}$ такой, что $\ln \left(\bar{s}_{1}^{\prime}\right)=k$ и $\left(\mathcal{S}_{d}\right)_{\bar{s}_{1}^{\prime}}=\mathcal{S}_{d}$. Следовательно, согласно индуктивному предположению имеем

$$
s=x_{\sigma_{1,0}} \cdot\left(x_{\sigma_{2}^{\prime \prime}} \cdot \ldots \cdot x_{\sigma_{m_{0}}^{\prime \prime}} \cdot \bar{s}_{1}^{\prime}\right)=x_{\sigma_{1,0}} \cdot\left(x_{\sigma_{2,0}} \cdot \ldots \cdot x_{\sigma_{m_{0}, 0}} \cdot \bar{s}_{1}^{\prime \prime}\right)
$$

где элемент $\bar{s}_{1}^{\prime \prime} \in S_{d}$ такой, что $\ln \left(\bar{s}_{1}^{\prime \prime}\right)=k$ и $\left(\mathcal{S}_{d}\right)_{\bar{s}_{1}^{\prime \prime}}=\mathcal{S}_{d}$. Согласно предложению 2.4 имеем $\bar{s}_{1}^{\prime \prime}=r\left(x_{\sigma}\right) \cdot h_{d, g}$, где $\sigma=\alpha\left(\bar{s}_{1}^{\prime \prime}\right)=\left(\sigma_{1,0} \ldots \sigma_{m, 0}\right)^{-1} \alpha(s)$ и $g=\frac{k-\ln _{t}\left(x_{\sigma}\right)}{2}-d+1$. Теорема доказана.

СлеДСтвиЕ 2.2. Пусть $s_{i}=x_{\sigma_{1, i}} \cdot \ldots \cdot x_{\sigma_{m, i}} \cdot \bar{s}_{i}, i=1,2,-$ два элемента полугруппы $\Sigma_{d}^{\mathcal{S}_{d}}$, где элементы $\bar{s}_{i} \in S_{T_{d}}$ имеют длину $\ln \left(\bar{s}_{1}\right)=\ln \left(\bar{s}_{2}\right)=k$. Предположим, что $\alpha\left(s_{1}\right)=\alpha\left(s_{2}\right)$ u $\tau\left(s_{1}\right)=\tau\left(s_{2}\right)$. Если $k \geqslant 3(d-1)$, то $s_{1}=s_{2}$. 
СледствиЕ 2.3. Элемент Гурвища $h_{d,\left[\frac{d}{2}\right]}$ является стабилизирующим элементом полугруппы $\Sigma_{d}, u$, следовательно, $\Sigma_{d}$ является стабильной полугрупnоน̆.

Также имеет место однозначность разложения на множители единицы в $\mathcal{S}_{d}$ и в следующем случае.

TеОрема 2.4 [9]. Пусть $s=s_{1} \cdot s_{2} u s^{\prime}=s_{1}^{\prime} \cdot s_{2}^{\prime} \in \Sigma_{d, \mathbf{1}}$ - такие элементы, что $s_{1}, s_{1}^{\prime} \in S_{T_{d}} u$ группь $\left(\mathcal{S}_{d}\right)_{s} u\left(\mathcal{S}_{d}\right)_{s^{\prime}}$ действуют транзитивно на множестве $I_{d}$. Тогда если $\tau(s)=\tau\left(s^{\prime}\right) u \ln \left(s_{2}\right)=\ln \left(s_{2}^{\prime}\right) \leqslant 2$, mо $s=s^{\prime}$.

Однако, как показывает следующий пример, теорема 2.4 становится неверной даже в случае $s, s^{\prime} \in \Sigma_{d, \mathbf{1}}^{\mathcal{S}_{d}}$, если $\ln \left(s_{2}\right)=\ln \left(s_{2}^{\prime}\right)>2$.

ПримеР 2.1 [9]. Рассмотрим перестановки $\sigma_{1}=\sigma_{1}^{\prime}=(1,2,3)(5,6,7,8)$, $\sigma_{2}=(1,2)(3,4,5), \sigma_{3}=\left(\sigma_{1} \sigma_{2}\right)^{-1}=(8,7,6,5,4,2,3)$ и $\sigma_{2}^{\prime}=(7,8)(3,4,5)$, $\sigma_{3}^{\prime}=\left(\sigma_{1}^{\prime} \sigma_{2}^{\prime}\right)^{-1}=(8,6,5,4,2,1,3)$ в $\mathcal{S}_{8}$. Тогда элементы $s=x_{\sigma_{1}} \cdot x_{\sigma_{2}} \cdot x_{\sigma_{3}}$ и $s^{\prime}=x_{\sigma_{1}^{\prime}} \cdot x_{\sigma_{2}^{\prime}} \cdot x_{\sigma_{3}^{\prime}} \in \Sigma_{8,1}^{\mathcal{S}_{8}}$ имеют один и тот же тип, но $s \neq s^{\prime}$.

2.5. Разложения в группе $\mathcal{S}_{3}$. Рассмотрим полугруппу $\Sigma_{3,1} \subset \Sigma_{3}$. Полугруппа $\Sigma_{3}$ порождается элементами $x_{(1,2)}, x_{(1,3)}, x_{(2,3)}, x_{(1,2,3)}$ и $x_{(1,3,2)}$, связанными следующими соотношениями:

$$
\begin{gathered}
x_{(1,2)} \cdot x_{(1,3)}=x_{(2,3)} \cdot x_{(1,2)}=x_{(1,3)} \cdot x_{(2,3)}, \\
x_{(1,3)} \cdot x_{(1,2)}=x_{(2,3)} \cdot x_{(1,3)}=x_{(1,2)} \cdot x_{(2,3)}, \\
x_{(1,2)} \cdot x_{(1,2,3)}=x_{(1,3,2)} \cdot x_{(1,2)}=x_{(1,3)} \cdot x_{(1,3,2)}=x_{(1,2,3)} \cdot x_{(1,3)}, \\
x_{(1,2)} \cdot x_{(1,3,2)}=x_{(1,2,3)} \cdot x_{(1,2)}=x_{(2,3)} \cdot x_{(1,2,3)}=x_{(1,3,2)} \cdot x_{(2,3)}, \\
x_{(2,3)} \cdot x_{(1,3,2)}=x_{(1,2,3)} \cdot x_{(2,3)}=x_{(1,3)} \cdot x_{(1,2,3)}=x_{(1,3,2)} \cdot x_{(1,3)}, \\
x_{(1,2,3)} \cdot x_{(1,3,2)}=x_{(1,3,2)} \cdot x_{(1,2,3)} .
\end{gathered}
$$

Обозначим

$$
\begin{gathered}
s_{1}=x_{(1,2)}^{2}, \quad s_{2}=x_{(2,3)}^{2}, \quad s_{3}=x_{(1,3)}^{2}, \quad s_{4}=x_{(1,2,3)} \cdot x_{(1,3,2)}, \\
s_{5}=x_{(1,2,3)} \cdot x_{(1,3)} \cdot x_{(2,3)}, \quad s_{6}=x_{(1,2,3)}^{3}, \quad s_{7}=x_{(1,3,2)}^{3} .
\end{gathered}
$$

Легко видеть, что $s_{1}, \ldots, s_{7} \in \Sigma_{3,1}$.

Теорема 2.5. Полугруппа $\Sigma_{3,1}$ имеет следующее копредставление:

$$
\begin{aligned}
\Sigma_{3,1}=\left\{s_{1}, \ldots, s_{7}\right. & \mid s_{i} \cdot s_{j}=s_{j} \cdot s_{i}, 1 \leqslant i, j \leqslant 7 ; \\
& s_{i} \cdot s_{k}=s_{j} \cdot s_{k}, 1 \leqslant i, j \leqslant 3,4 \leqslant k \leqslant 7 ; \\
& s_{i} \cdot s_{6}=s_{i} \cdot s_{7}, 1 \leqslant i \leqslant 3 ; \\
& s_{1} \cdot s_{2}=s_{1} \cdot s_{3}=s_{2} \cdot s_{3} ; \\
& s_{4}^{3}=s_{6} \cdot s_{7} ; s_{5}^{2}=s_{1}^{2} \cdot s_{4} ; s_{5}^{3}=s_{1}^{3} \cdot s_{6} ; \\
& \left.s_{4} \cdot s_{5}=s_{1} \cdot s_{6}=s_{1} \cdot s_{7}\right\} .
\end{aligned}
$$

ДокАЗАтЕльство. Прежде всего покажем, что элементы $s_{1}, \ldots, s_{7}$ порождают полугруппу $\Sigma_{3,1}$. Действительно, предположим, что любой элемент $s \in \Sigma_{3,1}$ длины $\ln (s) \leqslant k$ может быть выражен через элементы $s_{1}, \ldots, s_{7}$, и 
рассмотрим элемент $s \in \Sigma_{3,1}$ длины $\ln (s)=k+1$. Передвинув множители $x_{(1,2,3)}$ и $x_{(1,3,2)}$ влево, любой элемент $s \in \Sigma_{3,1}$ можем записать в следующем виде:

$$
s=x_{(1,2,3)}^{a} \cdot x_{(1,3,2)}^{b} \cdot s^{\prime},
$$

где $a, b$ - неотрицательные целые числа и $s^{\prime}-$ слово, состоящее из букв $x_{(1,2)}$, $x_{(1,3)}$ и $x_{(2,3)}$.

Согласно леммам 2.1, 2.2 если $\ln \left(s^{\prime}\right) \geqslant 3$, то $s^{\prime}$ может быть записан в виде $s^{\prime}=x_{(i, j)}^{2} \cdot s^{\prime \prime}$. Аналогично, если либо $a \geqslant 3$, либо $b \geqslant 3$, либо оба $a$ и $b$ положительны, то $s=s_{i} \cdot \widetilde{s}$, где $i$ - это либо 6 , либо 7 , либо 4 и $\widetilde{s} \in \Sigma_{3, \mathbf{1}}$, $\ln (\widetilde{s}) \leqslant k-1$. Таким образом, нам необходимо рассмотреть только случаи, когда $\ln \left(s^{\prime}\right) \leqslant 2$ и либо $0 \leqslant a \leqslant 2, b=0$, либо $a=0,0 \leqslant b \leqslant 2$. Если $a=b=0$, то очевидно, что $s^{\prime}=s_{i}$ для некоторого $i=1,2,3$, так как $s=s^{\prime} \in \Sigma_{3, \mathbf{1}}$.

Рассмотрим случай $a=1$ и $b=0$, т. е. $s=x_{(1,2,3)} \cdot s^{\prime}$. Поскольку $s \in \Sigma_{3,1}$ и $\alpha\left(x_{(1,2,3)}\right)=(1,2,3)$, мы имеем $\alpha\left(s^{\prime}\right)=(1,3,2)$. Следовательно, $s^{\prime}$ равен либо $x_{(1,3)} \cdot x_{(2,3)}$, либо $x_{(2,3)} \cdot x_{(1,2)}$, либо $x_{(1,2)} \cdot x_{(1,3)}$. Однако согласно соотношениям (2.11) последние три элемента равны друг другу, и в этом случае $s=s_{5}$.

Аналогично, если $a=0, b=1$, т. е. $s=x_{(1,3,2)} \cdot s^{\prime}$, то мы получим, что $s^{\prime}$ равен либо $x_{(1,2)} \cdot x_{(2,3)}$, либо $x_{(2,3)} \cdot x_{(1,3)}$, либо $x_{(1,3)} \cdot x_{(1,2)}$, и согласно соотношениям (2.12) последние три элемента равны друг другу. Следовательно, согласно (2.13) имеем

$$
\begin{aligned}
s & =x_{(1,3,2)} \cdot x_{(1,2)} \cdot x_{(2,3)}=x_{(1,2)} \cdot x_{(1,2,3)} \cdot x_{(2,3)} \\
& =x_{(1,2,3)} \cdot x_{(1,3)} \cdot x_{(2,3)}=s_{5} .
\end{aligned}
$$

Если $a=2, b=0$, т. е. $s=x_{(1,2,3)}^{2} \cdot s^{\prime}$, то мы получаем, что $\alpha\left(s^{\prime}\right)=(1,2,3)$, и поэтому $s^{\prime}=x_{(1,2)} \cdot x_{(2,3)}$. Следовательно, согласно соотношениям $(2.14)$ имеем

$$
\begin{aligned}
s & =x_{(1,2,3)}^{2} \cdot x_{(1,2)} \cdot x_{(2,3)}=x_{(1,2,3)} \cdot x_{(1,2)} \cdot x_{(1,3,2)} \cdot x_{(2,3)} \\
& =x_{(1,2,3)} \cdot x_{(1,3,2)} \cdot x_{(2,3)} \cdot x_{(2,3)}=s_{4} \cdot s_{2} .
\end{aligned}
$$

Наконец, если $a=0, b=2$, т. е. $s=x_{(1,3,2)}^{2} \cdot s^{\prime}$, то мы имеем $\alpha\left(s^{\prime}\right)=(1,3,2)$, и поэтому $s^{\prime}=x_{(1,3)} \cdot x_{(2,3)}$. Следовательно, согласно соотношениям (2.15) имеем

$$
\begin{aligned}
s & =x_{(1,3,2)}^{2} \cdot x_{(1,3)} \cdot x_{(2,3)}=x_{(1,3,2)} \cdot x_{(1,3)} \cdot x_{(1,2,3)} \cdot x_{(2,3)} \\
& =x_{(1,3,2)} \cdot x_{(1,2,3)} \cdot x_{(2,3)} \cdot x_{(2,3)}=s_{4} \cdot s_{2},
\end{aligned}
$$

тем самым мы показали, что $\Sigma_{3,1}$ порождается элементами $s_{1}, \ldots, s_{7}$.

Поскольку проверка того, что порождающие элементы $s_{1}, \ldots, s_{7}$ полугруппы $\Sigma_{3,1}$ удовлетворяют всем соотношениям, упомянутым в формулировке теоремы 2.5, аналогична для всех соотношений, мы проверим только одно из них.

Покажем, например, что $s_{4} \cdot s_{5}=s_{6} \cdot s_{1}$. Согласно соотношениям (2.11)-(2.16) имеем

$$
\begin{aligned}
& s_{4} \cdot s_{5}=x_{(1,2,3)} \cdot \underline{x_{(1,3,2)} \cdot x_{(1,2,3)}} \cdot x_{(1,3)} \cdot x_{(2,3)} \\
& =x_{(1,2,3)} \cdot\left(x_{(1,2,3)} \cdot x_{(1,3,2)}\right) \cdot x_{(1,3)} \cdot x_{(2,3)} \\
& =x_{(1,2,3)} \cdot x_{(1,2,3)} \cdot\left(x_{(1,2,3)} \cdot x_{(2,3)}\right) \cdot x_{(2,3)}=x_{(1,2,3)} \cdot x_{(1,2,3)} \cdot x_{(1,2,3)} \cdot x_{(2,3)}^{2}
\end{aligned}
$$




$$
\begin{aligned}
& =x_{(1,2,3)} \cdot x_{(1,2,3)} \cdot \underline{\left(x_{(1,3)}^{2} \cdot x_{(1,2,3)}\right)}=x_{(1,2,3)} \cdot x_{(1,2,3)} \cdot\left(x_{(1,2,3)} \cdot x_{(1,2)}^{2}\right) \\
& =s_{6} \cdot s_{1} .
\end{aligned}
$$

Утверждение о том, что соотношения, упомянутые в формулировке теоремы 2.5, являются определяющими, вытекает из следующей теоремы.

ТеОрема 2.6. Каждый элемент $s \in \Sigma_{3,1}, s \neq \mathbf{1}$, имеет нормальную форму, т.е. он равен одному и только одному элементу одного из следующих видов:

$$
s= \begin{cases}s_{i}^{n}, & i=1,2,3, n \in \mathbb{N}, \\ s_{4}^{a} \cdot s_{6}^{m} \cdot s_{7}^{n}, & 0 \leqslant a \leqslant 2, m \geqslant 0, n \geqslant 0, a+m+n>0, \\ s_{1}^{n} \cdot s_{2}, & n \in \mathbb{N}, \\ s_{1}^{n} \cdot s_{6}^{m}, & m, n \in \mathbb{N}, \\ s_{1}^{n} \cdot s_{6}^{m} \cdot s_{4}, & m \geqslant 0, n>0, \\ s_{1}^{n} \cdot s_{6}^{m} \cdot s_{5}, & m \geqslant 0, n \geqslant 0 .\end{cases}
$$

ДокАЗАТЕЛЬСтво. Если $s \notin \Sigma_{3,1}^{\mathcal{S}_{3}}$, то очевидно, что $s$ равен либо $s_{i}^{n}, i=$ $1,2,3$, либо $s_{4}^{a} \cdot s_{6}^{m} \cdot s_{7}^{n}$.

Пусть $s \in \Sigma_{3, \mathbf{1}}^{\mathcal{S}_{3}}$. Если $s \in S_{T_{3}, \mathbf{1}}$, то согласно теореме Клебша-Гурвица $s=h_{3, g}$ для некоторого $g$.

Пусть $s=s^{\prime} \cdot s^{\prime \prime}$, где $s^{\prime}=x_{(1,2,3)}^{k_{1}} \cdot x_{(1,3,2)}^{k_{2}}$ и $s^{\prime \prime} \in S_{T_{3}}$. Применив соотношения (2.13)-(2.16), мы можем предполагать, что $s^{\prime}=x_{(1,2,3)}^{k}$ для $k=k_{1}+k_{2}$. Если $k \equiv 0(\bmod 3)$, то, применив соотношения из теоремы 2.5 , получим $s=s_{1}^{n} \cdot s_{6}^{m}$. Если $k \equiv 1(\bmod 3)$, то $s^{\prime}=s_{6}^{m} \cdot x_{(1,2,3)}$ и $x_{(1,2,3)} \cdot s^{\prime \prime} \in \Sigma_{3, \mathbf{1}}$. Согласно теореме 2.5 имеем $x_{(1,2,3)} \cdot s^{\prime \prime}=s_{5} \cdot s_{1}^{n}$ при некотором $n \geqslant 0$. Аналогично, если $k \equiv 2(\bmod 3)$, то $s^{\prime}=s_{6}^{m} \cdot x_{(1,2,3)}^{2}$ и $x_{(1,2,3)}^{2} \cdot s^{\prime \prime} \in \Sigma_{3, \mathbf{1}}$. Применив соотношения $(2.13)-(2.16)$, получим $x_{(1,2,3)}^{2} \cdot s^{\prime \prime}=x_{(1,2,3)} \cdot x_{(1,3,2)} \cdot s_{1}^{\prime \prime}=s_{4} \cdot s_{1}^{\prime \prime}$ с некоторым $s_{1}^{\prime \prime} \in S_{T_{3}, \mathbf{1}}$, а применив соотношения из теоремы 2.5 , получим $s=s_{1}^{n} \cdot s_{4} \cdot s_{6}^{m}$.

Для завершения доказательства осталось заметить, что различные нормальные формы определяют различные элементы, так как они имеют различные инварианты $G_{s}$ и $\tau(s) \in \mathbb{Z}_{\geqslant 0}^{2}$.

ТеОрема 2.7. С точностъю до одновременного сопряжения элемент $\bar{s} \in \Sigma_{3}$ равен либо элементу $s$, дде $s$ - один из элементов полугруппы $\Sigma_{3, \mathbf{1}}$, описанных в теореме 2.6, либо элементу

$$
\bar{s}= \begin{cases}x_{(1,2)}^{2 k+1}, & k \geqslant 0, \\ x_{(1,2,3)}^{n} \cdot x_{(1,3,2)}^{m}, & n>m, n-m \neq 0(\bmod 3), \\ x_{(1,2)}^{n} \cdot x_{(2,3)}, & n \in \mathbb{N}, \\ x_{(1,2)}^{n} \cdot x_{(1,2,3)}^{3 m} \cdot x_{(1,3,2)}^{a}, & n \in \mathbb{N}, m \geqslant 0, a=0,1,2, \\ & u a \neq 0, \text { ecлu } n \equiv 0(\bmod 2) .\end{cases}
$$

ЗАмечАниЕ 2.2. Элементы $s_{1}^{n}, s_{2}^{n}$ и $s_{3}^{n}$ из теоремы 2.6 сопряжены друг другу. Элементы $s_{4}^{a} \cdot s_{6}^{m} \cdot s_{7}^{n}$ и $s_{4}^{a} \cdot s_{6}^{n} \cdot s_{7}^{m}$ также являются сопряженными.

ДокАЗАТЕЛЬСтво тЕОРЕмы 2.7. Чтобы доказать теорему, мы должны рассмотреть отдельно следующие случаи: 
1) $\left(\mathcal{S}_{3}\right)_{s}=\mathcal{S}_{2}$

2) $\left(\mathcal{S}_{3}\right)_{s}=A_{3}$, где $A_{3}$ - знакопеременная группа;

3) $s \in S_{T_{3}},\left(\mathcal{S}_{3}\right)_{s}=\mathcal{S}_{3}$, и $\alpha(s)$ - либо транспозиция, либо циклическая перестановка длины 3 ;

4) $s \notin S_{T_{3}},\left(\mathcal{S}_{3}\right)_{s}=\mathcal{S}_{3}$, и $\alpha(s)$ - либо транспозиция, либо циклическая перестановка длины 3.

Легко видеть, что в случаях 1)-3) элемент $s$ равен (с точностью до сопряжения) соответственно одному из элементов $x_{(1,2)}^{2 k+1}, x_{(1,2,3)}^{n} \cdot x_{(1,3,2)}^{m}, x_{(1,2)}^{n} \cdot x_{(2,3)}$.

В случае 4) имеем $s=s_{1} \cdot s_{2}, s_{1} \in S_{T_{d}}$ и $s_{2}$ представлен словом, составленным из букв $x_{(1,2,3)}$ и $x_{(1,3,2)}$. Согласно соотношениям $(2.13)$ и (2.14) мы можем предполагать, что $s_{1}=x_{(1,2)}^{n}$. Кроме того, имеем

$$
x_{(1,2)} \cdot x_{(1,2,3)}^{3}=x_{(1,3,2)}^{3} \cdot x_{(1,2)}=x_{(1,2)} \cdot x_{(1,3,2)}^{3} .
$$

Применив эти соотношения и $(2.16)$, получим, что $s=x_{(1,2)}^{n} \cdot x_{\sigma}^{3 m} \cdot x_{\sigma^{-1}}^{a}$, где $\sigma=(1,2,3)$ или $\sigma=(1,3,2)$. Чтобы завершить доказательство, заметим, что $\lambda((1,2))\left(x_{\sigma}\right)=x_{\sigma^{-1}}$.

СлЕДСТвИЕ 2.4. Пусть $\left(\mathcal{S}_{3}\right)_{s}=\mathcal{S}_{2}$ либо $\left(\mathcal{S}_{3}\right)_{s}=\mathcal{S}_{3}$ для $s \in \Sigma_{3}$. Тогда $s$ с точностью до одновременного сопряжения однозначно определяется своим типом $\tau(s)$ и типом $t(\alpha(s))$ своего образа $\alpha(s) \in \mathcal{S}_{3}$. C точностъю до одновременного сопряжения существует ровно $\left[\frac{n}{6}\right]+1$ различных элементов $s \in \Sigma_{3,1}^{A_{3}}$ длинь $\ln (s)=n$, если $n \not \equiv 1(\bmod 6)$, и если $n \equiv 1(\bmod 6)$, то существует ровно $\left[\frac{n}{6}\right]$ различных элементов $s \in \Sigma_{3,1}^{A_{3}}$ длины $\ln (s)=n$. Если $\alpha(s) \neq \mathbf{1}$, то существует ровно $m=-\left[\frac{-n}{3}\right]$ различных элементов $s \in \Sigma_{3}^{A_{3}}$ длины $\ln (s)=n$.

2.6. Вложения Кэли. Как известно, любая конечная группа $G$ может быть вложена в некоторую симметрическую группу. В частности, если $N=$ $|G|$ - порядок группы $G$, то мы имеем вложение Кэли $c: G \hookrightarrow \operatorname{Sym}(G) \simeq \mathcal{S}_{N}$ :

$$
\left(g_{1}\right) \sigma_{g}=g_{1} g, \quad g, g_{1} \in G, \quad c(g)=\sigma_{g},
$$

т. е. группа $G$ действует сама на себе умножениями справа. Отождествим группу $G$ с ее образом $c(G)$ и обозначим через $N(G)$ и $C(G)$ соответственно нормализатор и централизатор группы $G$ в $\mathcal{S}_{N}$. Поскольку $N(G)$ действует на группе $G$ сопряжениями, мы имеем естественный гомоморфизм $a: N(G) \rightarrow \operatorname{Aut}(G)$.

ТеОрема 2.8. Пусть $c: G \hookrightarrow \operatorname{Sym}(G) \simeq \mathcal{S}_{N}-$ вложение Кэли конечной группы $G$. Тогда естественный гомоморфизм $а: N(G) \rightarrow \operatorname{Aut}(G)$ имеет следующие свойства:

(i) а является эпиморфизмом;

(ii) $\operatorname{ker} a=C(G) \simeq G$;

(iii) группа, порожденная элементами групп $G$ и $C(G)$, изоморфна прямому произведению $G \times_{C} G$ с обгединенной подгруппой $C$, где $C$ - иентр группы $G$.

ДокАЗАтЕЛЬСтво. Рассмотрим автоморфизм $f \in \operatorname{Aut}(G)$ как перестановку $\sigma_{f} \in \mathcal{S}_{N}$ элементов группы $G$ :

$$
(g) \sigma_{f}=f(g), \quad g \in G .
$$


Покажем, что $\sigma_{f} \in N(G)$. Для всех $g_{1} \in G$ имеем

$$
\begin{aligned}
\left(g_{1}\right) \sigma_{f}^{-1} \sigma_{g} \sigma_{f} & =\left(f^{-1}\left(g_{1}\right)\right) \sigma_{g} \sigma_{f}=\left(f^{-1}\left(g_{1}\right) g\right) \sigma_{f} \\
& =f\left(f^{-1}\left(g_{1}\right) g\right)=g_{1} f(g)=\left(g_{1}\right) \sigma_{f(g)},
\end{aligned}
$$

т. е. $\sigma_{f}^{-1} \sigma_{g} \sigma_{f}=\sigma_{f(g)} \in G$ для всех $g \in G$. Поэтому $\sigma_{f} \in N(G)$ и, более того, сопряжение элементов из $G$ на $\sigma_{f}$ определяет автоморфизм $f$ группы $G$. Следовательно, гомоморфизм $а$ является эпиморфизмом.

Очевидно, что $C(G)=\operatorname{ker} a$. Рассмотрим элемент $\sigma \in C(G)$. Имеем $\sigma_{g} \sigma=$ $\sigma \sigma_{g}$ для всех $g \in G$. Следовательно,

$$
\left(g_{1}\right) \sigma_{g} \sigma=\left(g_{1} g\right) \sigma=\left(\left(g_{1}\right) \sigma\right) \cdot g
$$

для всех $g_{1}, g \in G$. В частности, если для $g_{1}=\mathbf{1}$ мы обозначим $(\mathbf{1}) \sigma$ через $g_{\sigma}$, то будем иметь

$$
\text { (1) } \sigma_{g} \sigma=(g) \sigma=g_{\sigma} g
$$

для всех $g \in G$. Равенство $(g) \sigma=g_{\sigma} g$ показывает, что $\sigma$ действует на группе $G$ как умножение с левой стороны на элемент $g_{\sigma} \in G$. Очевидно, что умножения в группе $G$ слева и справа коммутируют друг с другом. Следовательно, $C(G) \simeq G$.

Напомним, что согласно определению группа $G$ действует на себе умножениями справа. Отсюда легко видеть, что группа, порожденная элементами групп $G$ и $C(G)$, изоморфна прямому произведению $G \times_{C} G$ с объединенной подгруппой, где $C$ - центр группы $G$.

Любое вложение группы $G \hookrightarrow \mathcal{S}_{d}$ определяет вложение полугрупп $S(G, O) \hookrightarrow$ $\Sigma_{d}$. Пусть $c: S_{G}=S(G, G) \hookrightarrow \Sigma_{d}-$ вложение полугрупп, определенное вложением Кэли $c: G \rightarrow \mathcal{S}_{N}$. Из теоремы 2.8 вытекает

СлеДСтвиЕ 2.5. Орбиты действия сопряжениями группы $\mathcal{S}_{N}$ на $\Sigma_{N}$, nересекающие полугруппу $S(G, G)$, находятся во взаимно однозначном соответствии с орбитами действия группъ $\operatorname{Aut}(G)$ на $S(G, G)$.

\section{§ 3. Пространства Гурвица}

3.1. Отмеченные римановы поверхности. Пусть $f: C \rightarrow D_{R}=\{z \in \mathbb{C} \mid$ $|z| \leqslant R\}$ - риманова поверхность, т. е. $f$ - непрерывное конечное собственное разветвленное накрытие диска $D_{R}=\{|z| \leqslant R\}$ (или проективной прямой $\mathbb{P}^{1}$, если $R=\infty$ ) степени $d$, разветвленное в конечном числе точек, лежащих в $D_{R}^{0}=D_{R} \backslash \partial D_{R}=\{|z|<R\}$ (мы не предполагаем здесь, что $C$ обязательно является связной поверхностью). Говорят, что два накрытия $\left(C^{\prime}, f^{\prime}\right)$ и $\left(C^{\prime \prime}, f^{\prime \prime}\right)$ диска $D_{R}$ изоморфны, если существует гомеоморфизм $h: C^{\prime} \rightarrow C^{\prime \prime}$, сохраняющий ориентацию и такой, что $f^{\prime}=h \circ f^{\prime \prime}$, и говорят, что эти накрытия эквивалентны, если существуют сохраняющие ориентации гомеоморфизмы $\psi: D_{R} \rightarrow D_{R}$ и $\varphi: C^{\prime} \rightarrow C^{\prime \prime}$ такие, что $\psi$ оставляет неподвижными точки границы $\partial D_{R}$ диска $D_{R}$ и выполнено равенство $\psi \circ f^{\prime}=f^{\prime \prime} \circ \varphi$. Обозначим через $\mathcal{R}_{R, d}$ множество классов эквивалентных накрытий диска $D_{R}$ степени $d$ относительно определенного выше отношения эквивалентности. 
Пусть $q_{1}, \ldots, q_{b} \in D_{R}^{0}$ - точки, над которыми разветвлено накрытие $f$. Зафиксируем точку $o=o_{R}=e^{\frac{3}{2} \pi i} R \in \partial D_{R}$ (если $R=\infty$, то по определению $\left.o_{\infty}=\infty=\mathbb{P}^{1} \backslash \mathbb{C}\right)$ и перенумеруем точки из $f^{-1}(o)$. Нумерация точек прообраза $f^{-1}(o)$ определяет порядок в множестве $f^{-1}(o)$. Такие накрытия $(C, f)$ с фиксированной точкой $o \in D_{R}$ и фиксированным порядком на множестве точек прообраза $f^{-1}($ o) будем называть накрытиями с упорядоченным множеством листов, или отмеченными накрытиями. Скажем, что отмеченные накрытия $\left(C^{\prime}, f^{\prime}\right)_{m}$ и $\left(C^{\prime \prime}, f^{\prime \prime}\right)_{m}$ являются эквивалентными, если существуют сохраняющие ориентации гомеоморфизмы $\psi: D_{R} \rightarrow D_{R}$ и $\varphi: C^{\prime} \rightarrow C^{\prime \prime}$ такие, что:

(i) $\psi$ оставляет неподвижными точки из $\partial D_{R}$;

(ii) $\varphi\left(p_{i}^{\prime}\right)=p_{i}^{\prime \prime} \in\left(f^{\prime \prime}\right)^{-1}(o)$ для каждой точки $p_{i}^{\prime} \in\left(f^{\prime}\right)^{-1}(o), i=1, \ldots, d$;

(iii) $\psi \circ f^{\prime}=f^{\prime \prime} \circ \varphi$.

Обозначим через $\mathcal{R}_{R, d}^{m}$ множество классов эквивалентных отмеченных накрытий диска $D_{R}$ степени $d$ относительно этого отношения эквивалентности. Перенумерация листов определяет действие симметрической группы $\mathcal{S}_{d}$ на множестве $\mathcal{R}_{R, d}^{m}$, и легко видеть, что $\mathcal{R}_{R, d}=\mathcal{R}_{R, d}^{m} / \mathcal{S}_{d}$.

Если $R_{1}<R_{2}<\infty$, то любое разветвленное накрытие $f: C \rightarrow D_{R_{1}}$ может быть продолжено до разветвленного накрытия $\tilde{f}: \widetilde{C} \rightarrow D_{R_{2}}$, не разветвленного над $D_{R_{2}} \backslash D_{R_{1}}$. Подъем пути

$$
l(t)=e^{\frac{3}{2} \pi i}\left(R_{2} t+(1-t) R_{1}\right) \subset D_{R_{2}} \backslash D_{R_{1}}^{0}, \quad t \in[0,1],
$$

в $\widetilde{C}$ дает $d$ путей $\tilde{f}^{-1}(l(t))$, соединяющих точки множества $f^{-1}\left(o_{R_{1}}\right)$ с точками из $f^{-1}\left(o_{R_{2}}\right)$. Если $(C, f)_{m}$ - отмеченное накрытие, то эти пути позволяют задать порядок на точках множества $f^{-1}\left(o_{R_{2}}\right)$, согласованный с имеющимся порядком на точках множества $f^{-1}\left(o_{R_{1}}\right)$. В результате мы получаем изоморфизм $i_{R_{1}, R_{2}}: \mathcal{R}_{R_{1}, d}^{m} \hookrightarrow \mathcal{R}_{R_{2}, d}^{m}$.

Аналогично, для любого отмеченного накрытия $(C, f)_{m}$ проективной прямой $\mathbb{P}^{1}$ и для любого $R>0$ найдется эквивалентное накрытие $(\bar{C}, \bar{f})_{m}$, точки ветвления которого лежат в $D_{R}^{0}$. Рассмотрим ограничение $\tilde{f}$ накрытия $\bar{f}$ на $\widetilde{C}=\bar{f}^{-1}\left(D_{R}\right)$. Если мы поднимем путь

$$
l(t)=e^{\frac{3}{2} \pi i} R / t \subset \mathbb{P}^{1} \backslash D_{R}^{0}, \quad t \in[0,1],
$$

в $\bar{C}$, то получим $d$ путей $\bar{f}^{-1}(l(t))$, соединяющих точки из множества $f^{-1}\left(o_{\infty}\right)$ с точками из $f^{-1}\left(o_{R}\right)$ и позволяющих определить порядок на точках множества $\tilde{f}^{-1}\left(o_{R}\right)$ по имеющемуся порядку на точках из $\bar{f}^{-1}\left(o_{\infty}\right)$. Очевидно, класс эквивалентности полученных отмеченных накрытий $(\widetilde{C}, \tilde{f})_{m}$ не зависит от выбора представителя $(\bar{C}, \bar{f})_{m}$. Следовательно, мы получаем вложение $i_{\infty, R}: \mathcal{R}_{\infty, d}^{m} \hookrightarrow \mathcal{R}_{R, d}^{m}$. Легко видеть, что $i_{\infty, R_{2}}=i_{R_{1}, R_{2}} \circ i_{\infty, R_{1}}$ для любых $R_{2} \geqslant R_{1}>0$. 
3.2. Полугруппы отмеченных накрытий. Петля $\gamma \subset D_{R} \backslash\left\{q_{1}, \ldots, q_{b}\right\}$ с началом и концом в точке $o=o_{R}$ может быть поднята в $C$ при помощи накрытия $f$, и мы получим $d$ путей, начинающихся и заканчивающихся в точках из $f^{-1}(o)$. Такой подъем петель определяет гомоморфизм (монодромию отмеченных накрытий) $\mu: \pi_{1}\left(D_{R} \backslash\left\{q_{1}, \ldots, q_{b}\right\}, o\right) \rightarrow \mathcal{S}_{d}$ в симметрическую группу $\mathcal{S}_{d}$ (монодромия отображает начальные точки поднятых путей в конечные точки тех же путей). Обратно, если задан гомоморфизм $\mu: \pi_{1}\left(D_{R} \backslash\left\{q_{1}, \ldots, q_{b}\right\}, o\right) \rightarrow \mathcal{S}_{d}$, то он определяет отмеченное накрытие $f: C \rightarrow D$ с монодромией $\mu$.

Фундаментальная группа $\pi_{1}\left(D_{R} \backslash\left\{q_{1}, \ldots, q_{b}\right\}, o\right)$ порождается петлями $\gamma_{1}, \ldots$ $\ldots, \gamma_{b}$ следующего вида. Каждая петля $\gamma_{i}$ состоит из пути $l_{i}$, начинающегося в точке $о$ и заканчивающегося в точке $q_{i}^{\prime}$, близкой к точке $q_{i}$, обхода в положительном направлении (относительно комплексной ориентации на $\mathbb{C}$ ) вдоль окружности $\Gamma_{i}$ малого радиуса с центром в $q_{i}, q_{i}^{\prime} \in \Gamma$, и последующего возврата в точку $q_{0}$ вдоль пути $l_{i}$ в обратном направлении; при $i \neq j$ петли $\gamma_{i}$ и $\gamma_{j}$ имеют только одну общую точку, а именно $о$; произведение $\gamma_{1} \ldots \gamma_{b}$ равно $\partial D_{R}$ в группе $\pi_{1}\left(D_{R} \backslash\left\{q_{1}, \ldots, q_{b}\right\}, o\right)$. Такой набор порождающих элементов называется хорошим геометрическим базисом группы $\pi_{1}\left(D_{R} \backslash\left\{q_{1}, \ldots, q_{b}\right\}, o\right)$. Хорошо известно, что если $R<\infty$, то $\gamma_{1}, \ldots, \gamma_{b}$ являются свободными порождающими группы $\pi_{1}\left(D_{R} \backslash\left\{q_{1}, \ldots, q_{b}\right\}, o\right)$, т. е. $\pi_{1}\left(D_{R} \backslash\left\{q_{1}, \ldots, q_{b}\right\}, o\right)=\left\langle\gamma_{1}, \ldots, \gamma_{b}\right\rangle$; и если $R=\infty$, то $\gamma_{1}, \ldots, \gamma_{b}$ порождают группу $\pi_{1}\left(\mathbb{P}^{1} \backslash\left\{q_{1}, \ldots, q_{b}\right\}, o\right)$ и связаны единственным соотношением $\gamma_{1} \ldots \gamma_{b}=\mathbf{1}$.

Если мы выберем хороший геометрический базис $\gamma_{1}, \ldots, \gamma_{b}$, то монодромия $\mu$ определяется набором элементов $\sigma_{1}=\mu\left(\gamma_{1}\right), \ldots, \sigma_{n}=\mu\left(\gamma_{b}\right) \in \mathcal{S}_{d}$, называемых локальными монодромиями, а произведение $\sigma=\sigma_{1} \ldots \sigma_{b}=\mu(\partial D)$ называется глобальной монодромией накрытия $f$. Легко видеть, что если $R=\infty$, то глобальная монодромия равна 1.

Набор $\left(\sigma_{1}, \ldots, \sigma_{b}\right)$ зависит от выбора хорошего геометрического базиса $\gamma_{1}, \ldots$ $\ldots, \gamma_{b}$. Любой хороший геометрический базис может быть получен из $\gamma_{1}, \ldots, \gamma_{b}$ с помощью конечного числа преобразований Гурвица. Другими словами, группа кос $\mathrm{Br}_{b}$ естественным образом действует на множестве хороших геометрических базисов группы $\pi_{1}\left(D_{R} \backslash\left\{q_{1}, \ldots, q_{b}\right\}, o\right)$ как преобразования Гурвица [10]. Следовательно, если $\left(\sigma_{1}^{\prime}, \ldots, \sigma_{b}^{\prime}\right)$ - набор, соответствующий другому геометрическому базису $\gamma_{1}^{\prime}, \ldots, \gamma_{b}^{\prime}$, то $\left(\sigma_{1}^{\prime}, \ldots, \sigma_{b}^{\prime}\right)$ может быть получен из набора $\left(\sigma_{1}, \ldots, \sigma_{b}\right)$ с помощью конечной последовательности преобразований Гурвица (см. п. 1.3).

Пусть $R<\infty$. На множестве $\mathcal{R}_{R, d}^{m}$ можно определить структуру полугруппы следующим образом. Пусть $\left(C_{1}, f_{1}\right)_{m}$ и $\left(C_{2}, f_{2}\right)_{m}$ - два отмеченных накрытия степени $d$. Выберем два непрерывных сохраняющих ориентацию вложения $\varphi_{j}: D_{R} \rightarrow D_{R}, j=1,2$, диска $D_{R}$ в себя, оставляющих неподвижной точку $о$ и таких, что:

(i) образ $\varphi_{1}\left(D_{R}\right)=\left\{u \in D_{R} \mid \operatorname{Re} u \geqslant 0\right\}$ является правым полудиском и $\varphi_{1}\left(\left\{u \in \partial D_{R} \mid \operatorname{Re} u \leqslant 0\right\}\right)=\left\{u \in D_{R} \mid \operatorname{Re} u=0\right\}$ - вертикальный диаметр;

(ii) $\varphi_{2}\left(D_{R}\right)=\left\{u \in D_{R} \mid \operatorname{Re} u \leqslant 0\right\}$ является левым полудиском и $\varphi_{2}(\{u \in$ $\left.\left.\partial D_{R} \mid \operatorname{Re} u \geqslant 0\right\}\right)=\left\{u \in D_{R} \mid \operatorname{Re} u=0\right\}$.

Отождествим точки, принадлежащие множествам $f_{1}^{-1}(o)$ и $f_{2}^{-1}(o)$, при помощи порядков, определенных на этих множествах, и после этого отождествим 
по непрерывности точки, принадлежащие $d$ путям $f_{1}^{-1}\left(\left\{u \in \partial D_{R} \mid \operatorname{Re} u \leqslant 0\right\}\right)$ в $C_{1}$ с точками, принадлежащими $d$ путям $f_{2}^{-1}\left(\left\{u \in \partial D_{R} \mid \operatorname{Re} u \geqslant 0\right\}\right)$ в $C_{2}$ так, чтобы образы всех отождествленных точек совпадали при отображениях $\varphi_{1} \circ f_{1}$ и $\varphi_{2} \circ f_{2}$. С помощью этих отождествлений мы можем склеить поверхности $C_{1}$ и $C_{2}$ вдоль этих $d$ путей и в результате получить отмеченное накрытие $(C, f)_{m}$, где $f(q)=\varphi_{1}\left(f_{1}(q)\right)$, если $q \in C_{1}$, и $f(q)=\varphi_{2}\left(f_{2}(q)\right)$, если $q \in C_{2}$. Назовем полученное накрытие $(C, f)_{m}$ произведением отмеченных накрытий $\left(C_{1}, f_{1}\right)_{m}$ и $\left(C_{2}, f_{2}\right)_{m}$ (обозначение: $\left.(C, f)_{m}=\left(C_{1}, f_{1}\right)_{m} \cdot\left(C_{2}, f_{2}\right)_{m}\right)$. Легко видеть, что введенное нами произведение определяет структуру некоммутативной полугруппы на множестве $\mathcal{R}_{R, d}^{m}$ такую, что отображения $i_{R_{1}, R_{2}}$ являются изоморфизмами полугрупп для всех $R_{1} \geqslant R_{2}>0$.

Очевидно, что полугруппа $\mathcal{R}_{d}^{m}=\mathcal{R}_{R, d}^{m}$ порождается отмеченными накрытиями $(C, f)_{m}$, которые являются накрытиями диска $D=D_{R}$ с одной только точкой ветвления $q_{1}$. Такие накрытия однозначно определяются (с точностью до эквивалентности) своей глобальной монодромией $\sigma_{f}=\mu(\partial D) \in \mathcal{S}_{d}$, где $\mu=\mu_{f}$ - монодромия отмеченного накрытия $(C, f)_{m}$. Следовательно, число порождающих элементов равно $d$ !. Обозначим через $x_{\sigma_{f}}$ порождающий элемент полугруппы $\mathcal{R}_{d}$, соответствующий накрытию $(C, f)_{m}$ с единственной точкой ветвления. Простая проверка показывает, что в полугруппе $\mathcal{R}_{d}^{m}$ порождающие элементы $x_{\sigma}$ удовлетворяют следующим определяющим соотношениям:

$$
x_{\sigma_{1}} \cdot x_{\sigma_{2}}=x_{\sigma_{2}} \cdot x_{\left(\sigma_{2}^{-1} \sigma_{1} \sigma_{2}\right)}, \quad x_{\sigma_{1}} \cdot x_{\sigma_{2}}=x_{\left(\sigma_{1} \sigma_{2} \sigma_{1}^{-1}\right)} \cdot x_{\sigma_{1}},
$$

и $x_{\sigma_{1}} \cdot x_{\mathbf{1}}=x_{\sigma_{1}}, x_{\mathbf{1}} \cdot x_{\sigma_{2}}=x_{\sigma_{2}}$ для всех $\sigma_{1}, \sigma_{2} \in \mathcal{S}_{d}$.

Легко проверить, что если отмеченное накрытие $(C, f)_{m}$ равно $x_{\sigma_{1}} \cdot \ldots \cdot x_{\sigma_{n}}$ в $\mathcal{R}_{d}^{m}$, то его глобальная монодромия $\sigma_{f}=\mu(\partial D)$ равна произведению $\sigma_{1} \ldots \sigma_{n}$ и очевидно, что сопоставление каждому отмеченному накрытию его глобальной монодромии определяет гомоморфизм из $\mathcal{R}_{d}^{m}$ в симметрическую группу $\mathcal{S}_{d}$. Обозначим этот гомоморфизм через $\alpha: \mathcal{R}_{d}^{m} \rightarrow \mathcal{S}_{d}$.

Перенумеровки листов в отмеченных накрытиях определяют действие группы $\mathcal{S}_{d}$ на $\mathcal{R}_{d}^{m}$. А именно, перестановка $\sigma_{0} \in \mathcal{S}_{d}$ действует на порождающих элементах $x_{\sigma}$ по следующему правилу: $x_{\sigma} \mapsto x_{\left(\sigma_{0}^{-1} \sigma \sigma_{0}\right)}$. Это действие определяет гомоморфизм $\lambda: \mathcal{S}_{d} \rightarrow \operatorname{Aut}\left(\mathcal{R}_{d}^{m}\right)$. Таким образом, мы получаем следующее

ПРЕДЛОЖеНИЕ 3.1. Полугруппа $\mathcal{R}_{d}^{m}$ как полугруппа над группой $\mathcal{S}_{d}$ ecmeственным образом изоморфна полугруппе $\Sigma_{d}$.

В соответствии с предложением 3.1 в дальнейшем элементы полугруппы $\Sigma_{d}$ будем называть разложениями на множители монодромий накрытий степени $d$.

Легко видеть, что ядро $\operatorname{ker} \alpha=\mathcal{R}_{d, \mathbf{1}}^{m}=\left\{(C, f)_{m} \in \mathcal{R}_{d}^{m} \mid \sigma_{f}=\mathbf{1}\right\}$ является подполугруппой полугруппы $\mathcal{R}_{d}^{m}$, оно изоморфно полугруппе $\Sigma_{d, 1}$ и если диск $D$ вложен в $\mathbb{P}^{1}$, то элементы полугруппы $\mathcal{R}_{d, \mathbf{1}}^{m}$ - это отмеченные накрытия $f: C \rightarrow D$, которые могут быть продолжены до отмеченных накрытий $\tilde{f}: \widetilde{C} \rightarrow \mathbb{C P}^{1}$, не разветвленных над $\mathbb{P}^{1} \backslash D$. Отметим, что такое продолжение $\tilde{f}: \widetilde{C} \rightarrow \mathbb{C P}^{1}$ отмеченного накрытия $f: C \rightarrow D$ с глобальной монодромией $\mu_{f}(\partial D)=\mathbf{1}$ определяется однозначно с точностью до эквивалентности. 
Обратное утверждение также верно: образ множества $\mathcal{R}_{\infty, d}^{m}$ при вложении $i_{\infty, R}$ совпадает с $\mathcal{R}_{d, \mathbf{1}}^{m}$. Ниже мы будем отождествлять множество $\mathcal{R}_{\infty, d}^{m}$ с полугруппой $\mathcal{R}_{d, \mathbf{1}}^{m}$ при помощи этого изоморфизма. В итоге мы получаем следующее

ПРЕДЛОЖЕНИЕ 3.2. На множестве классов эквивалентности отмеченных накрытий степени $d$ проективной прямой $\mathbb{P}^{1}$ имеется естественная структура полугруппы, изоморфной полугруппе $\Sigma_{d, \mathbf{1}}$.

\section{3. Пространства Гурвица отмеченных римановых поверхностей.}

В этом пункте мы опишем пространства Гурвица $\operatorname{HUR}_{d}^{m}(D)$ отмеченных разветвленных накрытий степени $d$ диска $D=D_{R}$, рассматриваемых с точностью до изоморфизма. Пространство $\operatorname{HUR}_{d}^{m}(D)=\bigsqcup_{b=0}^{\infty} \operatorname{HUR}_{d, b}^{m}(D)$ является несвязным объединением пространств накрытий, разветвленных в $b$ точках, $b \in \mathbb{N}$.

Как и в статье [3], рассмотрим симметрическое произведение $b$ копий открытого диска $D^{0}=D \backslash \partial D$ и обозначим его через $D^{(b)}$. Это комплексное многообразие размерности $b$, полученное как факторпространство произведения $D^{b}=D^{0} \times \cdots \times D^{0}($ с $b$ множителями $)$ при действии группы $\mathcal{S}_{b}$ перестановками множителей произведения. Точки пространства $D^{(b)}$ отождествляются с неупорядоченными наборами, состоящими из $b$ точек открытого диска $D^{0}$. Те из наборов $b$ точек, которые содержат менее $b$ различных точек, составляют дискриминантное множество $\Delta$ пространства $D^{(b)}$.

Для точки $B_{0}=\left\{q_{1,0}, \ldots, q_{b, 0}\right\} \in D^{(b)} \backslash \Delta$ зафиксируем упорядочение подмножества $B_{0}=\left\{q_{1,0}, \ldots, q_{b, 0}\right\} \subset D$ и выберем хороший геометрический базис $\gamma_{1}, \ldots, \gamma_{b}$ в группе $\pi_{1}\left(D \backslash B_{0}, o\right)$. Тогда любое слово $w$ из множества слов $W_{b}$ длины $b$, состоящих из букв $x_{\sigma}, \sigma \in \mathcal{S}_{d}$, определяет отмеченное накрытие $f=f_{w}: C \rightarrow D$, разветвленное над $B_{0}$, монодромия $\mu$ которого такова, что $\mu\left(\gamma_{i}\right)=\sigma_{i}$, где $x_{\sigma_{i}}$ - буква, стоящая на $i$-м месте в слове $w$.

Выбор хорошего геометрического базиса позволяет нам выбрать стандартные порождающие элементы $a_{1}, \ldots, a_{b-1}$ в группе $\pi_{1}\left(D^{(b)} \backslash \Delta, B_{0}\right) \simeq \mathrm{Br}_{b}$ таким образом, что этот выбор определяет действие группы $\mathrm{Br}_{b}$ на множестве слов $W_{b}$ (см. п. 1.3). Другими словами, этот выбор определяет гомоморфизм $\theta_{d, b, R}: \pi_{1}\left(D^{(b)} \backslash \Delta, B_{0}\right) \simeq \operatorname{Br}_{b} \rightarrow \mathcal{S}_{N}$, где $N=(d !)^{b}$.

Гомоморфизм $\theta_{d, b, R}$ позволяет нам определить пространство $\operatorname{HUR}_{d, b}^{m}(D)$ как неразветвленное накрытие $h_{d, b, R}: \operatorname{HUR}_{d, b}^{m}(D) \rightarrow D^{(b)} \backslash \Delta$, ассоциированное с гомоморфизмом $\theta_{d, b, R}$. Действительно, если мы зафиксируем отмеченное накрытие $f: C \rightarrow D$, монодромия $\mu$ которого такова, что $\mu\left(\gamma_{i}\right)=\sigma_{i}$, то любой путь $\delta(t), 0 \leqslant t \leqslant 1$, в $D^{(b)}$ с началом в точке $B_{0}$ может быть поднят в $D$ и мы получим $b$ путей $\delta_{i}(t)$ в $D$, начинающихся в точках $q_{1,0}, \ldots, q_{b, 0}$. Эти пути определяют (с точностью до изотопии) непрерывное семейство гомеоморфизмов $\bar{\delta}_{t}: D \backslash B_{0} \rightarrow D \backslash\left\{\delta_{1}(t), \ldots, \delta_{b}(t)\right\}$, оставляющих неподвижной точки границы $\partial D$, такое, что $\bar{\delta}_{0}=$ Id. Это семейство гомеоморфизмов определяет непрерывное семейство отмеченных накрытий $f_{t}: C_{t} \rightarrow D$, разветвленных в точках $\delta_{1}(t), \ldots, \delta_{b}(t)$ и заданных монодромией $\mu_{t}$ такой, что $\mu_{t}\left(\bar{\delta}_{t *}\left(\gamma_{i}\right)\right)=\sigma_{i}$. Очевидно, что если $\delta(t)$ - замкнутый путь, то набор $\left(\mu_{1}\left(\gamma_{1}\right), \ldots, \mu_{1}\left(\gamma_{b}\right)\right)$ гурвиц-эквивалентен набору $\left(\mu_{0}\left(\gamma_{1}\right), \ldots, \mu_{0}\left(\gamma_{b}\right)\right)$. Отсюда следует, что точки накрывающего пространства $\operatorname{HUR}_{d, b}^{m}(D)$ накрытия 
$h_{d, b, R}: \operatorname{HUR}_{d, b}^{m}(D) \rightarrow D^{(b)} \backslash \Delta$ естественным образом параметризуют все отмеченные накрытия диска $D$ степени $d$, разветвленные в $b$ точках. Степень накрытия $h_{d, b, R}$ равна $(d !)^{b}$. В итого мы получаем следующее

ПРЕДЛОЖЕНИЕ 3.3. Неприводимые компоненты пространства $\operatorname{HUR}_{d, b}^{m}(D)$ взаимно однозначно соответствуют элементам $s$ полугруппы $\Sigma_{d}$, длины которых $\ln (s)=b$. На множестве неприводимых компонент пространства $\operatorname{HUR}_{d}^{m}(D)$ имеется естественная структура полугруппь, изоморфная струкmуре полугруппь $\mathcal{R}_{d} \simeq \Sigma_{d}$.

Для $R_{2} \geqslant R_{1}>0$ мы имеем вложение $D_{R_{1}}^{(b)} \hookrightarrow D_{R_{2}}^{(b)}$ и легко видеть, что ограничение накрытия $h_{d, b, R_{2}}$ на $h_{d, b, R_{2}}^{-1}\left(D_{R_{1}}^{(b)} \backslash \Delta\right)$ может быть отождествлено с накрытием $h_{d, b, R_{1}}: \operatorname{HUR}_{d, b}^{m}\left(D_{R_{1}}\right) \rightarrow D_{R_{1}}^{(b)} \backslash \Delta$ при помощи отображения $i_{R_{1}, R_{2}}$.

В соответствии с предложением 3.3 обозначим через $\operatorname{HUR}_{d, s}^{m}(D)$ неприводимые компоненты пространства $\operatorname{HUR}_{d, \ln (s)}^{m}(D)$, соответствующие элементам $s \in \Sigma_{d}$. В частности, глобальная монодромия $\sigma_{f}=\mu(\partial D)=\alpha(s) \in \mathcal{S}_{d}$ является инвариантом неприводимой компоненты $\operatorname{HUR}_{d, s}^{m}(D)$. Положим

$$
\operatorname{HUR}_{d, b, \sigma}^{m}(D)=\bigcup_{\substack{\alpha(s)=\sigma \\ \ln (s)=b}} \operatorname{HUR}_{d, s}^{m}(D) .
$$

Из вышеизложенного следует, что

$$
\operatorname{HUR}_{d, b}^{m}\left(\mathbb{P}^{1}\right)=\bigcup_{R>0} \operatorname{HUR}_{d, b, \mathbf{1}}^{m}\left(D_{R}\right)
$$

Для фиксированного типа $t$ элементов $s \in \Sigma_{d}$ обозначим

$$
\operatorname{HUR}_{d, t}^{m}(D):=\bigcup_{\tau(s)=t} \operatorname{HUR}_{d, s}^{m}(D)
$$

и положим

$$
\operatorname{HUR}_{d, t, \sigma}^{m}(D)=\operatorname{HUR}_{d, t}^{m}(D) \cap \operatorname{HUR}_{d, \sigma}^{m}(D) .
$$

Как было упомянуто выше, отмеченное накрытие $f: C \rightarrow D$ степени $d$, разветвленное в точках $q_{1}, \ldots, q_{b}$, определяет монодромию $\mu: \pi_{1}\left(D \backslash\left\{q_{1}, \ldots, q_{b}\right\}\right) \rightarrow$ $\mathcal{S}_{d}$ (и определяется ею). Образ $\mu\left(\pi_{1}\left(D \backslash\left\{q_{1}, \ldots, q_{b}\right\}\right)\right)=\operatorname{Gal}(f) \subset \mathcal{S}_{d}$ называется группой Галуа накрытия $f$. Легко видеть, что $\operatorname{Gal}(f)=\left(\mathcal{S}_{d}\right)_{s}$, если накрытие $f$ принадлежит пространству $\operatorname{HUR}_{d, s}^{m}(D)$. Нетрудно показать, что накрывающее пространство $C$ отмеченного накрытия $(C, f)_{m}$ является связным тогда и только тогда, когда группа Галуа $\operatorname{Gal}(f)$ действует транзитивно на множестве $I_{d}=[1, d]$.

Обозначим через $\operatorname{HUR}_{d}^{m, G}(D)$ объединение неприводимых компонент пространства $\operatorname{HUR}_{d}^{m}(D)$, состоящих из накрытий с группой Галуа $\operatorname{Gal}(f)=G \subset \mathcal{S}_{d}$, и положим

$$
\begin{gathered}
\operatorname{HUR}_{d, t}^{m, G}(D)=\operatorname{HUR}_{d}^{m, G}(D) \cap \operatorname{HUR}_{d, t}^{m}(D), \\
\operatorname{HUR}_{d, t, \sigma}^{m, G}(D)=\operatorname{HUR}_{d, t}^{m, G}(D) \cap \operatorname{HUR}_{d, t, \sigma}^{m}(D) .
\end{gathered}
$$

Согласно следствию 2.2 верна 
Теорема 3.1. Пусть тип зиций. Если $k \geqslant 3(d-1)$, то каждая неприводимая компонента пространства $\mathrm{HUR}_{d, t}^{m, \mathcal{S}_{d}}(D)$ однозначно определяется глобальной монодромией $\sigma_{f}=\mu(\partial D) \in$ $\mathcal{S}_{d}$ накрытия $(C, f)_{m}$, принадлежащего этой неприводимой компоненте.

3.4. Пространства Гурвица (неотмеченных) накрытий диска. Чтобы получить пространство Гурвица $\operatorname{HUR}_{d, b}(D)$ накрытий диска $D=D_{R}$ степени $d$, разветвленных над $b$ точками, лежащими в $D^{0}$, нам надо отождествить все отмеченные накрытия диска $D$, отличающиеся друг от друга только нумерацией листов. Перенумерация листов индуцируется действием группы $\mathcal{S}_{d}$ на отмеченных слоях. Напомним, что действия групп $\operatorname{Br}_{b}$ и $\mathcal{S}_{d}$ на множестве $W_{b}$ коммутируют. Поэтому действие группы $\mathcal{S}_{d}$ индуцирует действие на пространстве $\operatorname{HUR}_{d, b}^{m}(D)$, и мы получаем, что пространство $\operatorname{HUR}_{d, b}(D)$ является факторпространством относительно этого действия: $\operatorname{HUR}_{d, b}(D)=\operatorname{HUR}_{d, b}^{m}(D) / \mathcal{S}_{d}$. Отсюда следует

ПрЕДЛОЖЕНИЕ 3.4. Неприводимые компоненты пространства $\operatorname{HUR}_{d, b}(D)$ находятся во взаимно однозначном соответствии с орбитами действия групnъь $\mathcal{S}_{d}$ одновременными сопряжениями на множестве $\Sigma_{d, b}=\left\{s \in \Sigma_{d} \mid\right.$ $\ln (s)=b\}$.

Если $f: C \rightarrow D$ - неотмеченное накрытие, то мы также можем определить группу Галуа накрытия как $\operatorname{Gal}(f)=\left(\mathcal{S}_{d}\right)_{s}$. Однако в этом случае подгруппа $\mathrm{Gal}(f)$ симметрической группы $\mathcal{S}_{d}$ определяется не однозначно, а только с точностью до внутренних автоморфизмов группы $\mathcal{S}_{d}$.

Ниже мы будем использовать следующие обозначения: HUR.,.,.(D) (соответственно, $\left.\mathrm{HUR}_{., ., .}^{G}(D)\right)$ - образ определенных выше подпространств

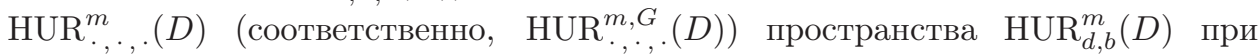
каноническом отображении

$$
\operatorname{HUR}_{d, b}^{m}(D) \rightarrow \operatorname{HUR}_{d, b}(D)=\operatorname{HUR}_{d, b}^{m}(D) / \mathcal{S}_{d} .
$$

В частности, имеем $\operatorname{HUR}_{d, s_{1}}(D)=\operatorname{HUR}_{d, s_{2}}(D)$ тогда и только тогда, когда существует такая перестановка $\sigma \in \mathcal{S}_{d}$, что $\lambda(\sigma)\left(s_{1}\right)=s_{2}$.

Следствие 2.4 дает полное описание неприводимых компонент пространства $\operatorname{HUR}_{d, b}(D)$ в случае $d=3$.

СлЕДСТВИЕ 3.1. Если $G \simeq \mathcal{S}_{2}$ или $G \simeq \mathcal{S}_{3}$, то неприводимые компоненты пространства $\operatorname{HUR}_{3, b}^{G}(D)$ однозначно определяются типом разложения на множители монодромии и типом глобальной монодромии. Если глобальная монодромия равна $\mathbf{1}$, то пространство $\operatorname{HUR}_{3, b}^{A_{3}}(D)$ coстоит из $m=\left[\frac{b}{6}\right]+1$ неприводимых компонент, если $b \not \equiv 1(\bmod 6)$, и состоит из $\left[\frac{b}{6}\right]$ неприводимых компонент, если $b \equiv 1(\bmod 6)$. Пространство $\operatorname{HUR}_{3, b}^{A_{3}}(D)$ состоит из $m=-\left[\frac{-b}{3}\right]$ неприводимых компонент, если глобальная монодромия не равна 1 .

3.5. Пространства Гурвица (неотмеченных) накрытий прямой $\mathbb{P}^{1}$. $\mathrm{B}$ работе [3] пространства Гурвица $\operatorname{HUR}_{d, b}\left(\mathbb{P}^{1}\right)$ накрытий проективной прямой $\mathbb{P}^{1}$ степени $d$, разветвленных над $b$ точками, были описаны как неразветвленные накрытия дополнения к дискриминантному множеству $\Delta$ в симметрическом произведении $\mathbb{P}^{(b)} b$ копий проективной прямой $\mathbb{P}^{1}$. Выбор точки 
$\infty \in \mathbb{P}^{1}$ и отождествление аффинной прямой $\mathbb{C}$ с $\mathbb{P}^{1} \backslash\{\infty\}$ определяет вложение пространства $\operatorname{HUR}_{d, b}\left(D_{\infty}\right)$ в $\mathrm{HUR}_{d, b}\left(\mathbb{P}^{1}\right)$ в виде открытого всюду плотного множества. Таким образом, мы получаем следующее

ПредлоЖениЕ 3.5. Неприводимые компоненты пространства $\mathrm{HUR}_{d, b}\left(\mathbb{P}^{1}\right)$ находятся во взаимно однозначном соответствии с орбитами действия групnъ $\mathcal{S}_{d}$ одновременными сопряжениями на множестве $\Sigma_{d, \mathbf{1}, \mathbf{b}}=\left\{s \in \Sigma_{d, \mathbf{1}} \mid\right.$ $\ln (s)=b\}$.

Kак и в п. 3.4, мы можем рассмотреть объединения HUR.,., $\left(\mathbb{P}^{1}\right)$ (соответственно, $\left.\operatorname{HUR}_{, ., .}^{G}\left(\mathbb{P}^{1}\right)\right)$ неприводимых компонент пространства $\operatorname{HUR}_{d, b}\left(\mathbb{P}^{1}\right)$ для фиксированных элементов полугруппы $\Sigma_{b, \mathbf{1}}$, для фиксированных типов разложений на множители монодромий, для фиксированных групп Галуа и т.д.

Как следствие из предложения 1.1, верна

Tеорема 3.2. На множестве неприводимых компонент пространства Гурвица $\mathrm{HUR}_{d}^{\mathcal{S}_{d}}\left(\mathbb{P}^{1}\right)$ существует естественная структура полугруппь $\Sigma_{d, \mathbf{1}}^{\mathcal{S}_{d}}=$ $\left\{s \in \Sigma_{d, 1} \mid\left(\mathcal{S}_{d}\right)_{s}=\mathcal{S}_{d}\right\}$.

Из теорем 2.3, 2.4 и следствия 2.4 получаем следующие теоремы.

Tеорема 3.3. Пространство $\operatorname{HUR}_{d, t}^{\mathcal{S}_{d}}\left(\mathbb{P}^{1}\right)$ неприводимо, если тип $t$ разложения на множители монодромии содержит не менее $3(d-1)$ транспозиций.

ТеОРема 3.4 [9]. Пусть $G$ - транзитивная подгруппа симметрической группы $\mathcal{S}_{d}$. Тогда пространство $\mathrm{HUR}_{d, t}^{G}\left(\mathbb{P}^{1}\right)$ неприводимо, если тип $t$ разложения на множители монодромии содержит не менее $l-2$ транспозиций, где l - длина типа монодромии (другими словами, $l$ - число точек ветвления накрытий).

ТеОРема 3.5. В случае $G \simeq \mathcal{S}_{2}$ или $G \simeq \mathcal{S}_{3}$ неприводимые компоненты пространства $\operatorname{HUR}_{3, b}^{G}\left(\mathbb{P}^{1}\right)$ однозначно определяются своим типом разложения на множители монодромии. Пространство $\mathrm{HUR}_{3, b}^{A_{3}}\left(\mathbb{P}^{1}\right)$ состоит из $m=\left[\frac{b}{6}\right]+1$ неприводимых компонент, если $b \not \equiv 1(\bmod 6)$, и состоит из $\left[\frac{b}{6}\right]$ неприводимых компонент, если $b \equiv 1(\bmod 6)$.

3.6. Пространства Гурвица накрытий Галуа. Пусть $f: C \rightarrow \mathbb{P}^{1}$ - накрытие Галуа с группой Галуа $G=\operatorname{Gal}\left(C / \mathbb{P}^{1}\right)$, т. е. $G$ является группой накрывающих преобразований накрытия $f$ и факторпространство $C / G$ совпадает с $\mathbb{P}^{1}$. В этом случае мы имеем $\operatorname{deg} f=|G|$, и если фиксирована точка $\infty \in \mathbb{P}^{1}$, над которой $f$ не разветвлено и, кроме того, фиксирована точка $e \in f^{-1}(\infty)$, то действие группы $G$ на слое $f^{-1}(\infty)$ определяет нумерацию точек из $f^{-1}(\infty)$ элементами группы $G$. Если мы перенумеруем точки, принадлежащие слою $f^{-1}(\infty)$, целыми числами отрезка $I_{|G|}=[1,|G|]$, то эти нумерации определяют вложение $G \hookrightarrow \mathcal{S}_{|G|}$. Легко видеть, что это вложение Кэли. Следовательно, пространство Гурвица $\operatorname{HUR}^{G}\left(\mathbb{P}^{1}\right)$ накрытий Галуа с группой Галуа $G$ может быть отождествлено с пространством $\operatorname{HUR}_{|G|, \mathbf{1}}^{G}\left(\mathbb{P}^{1}\right)$, и, в частности, естественное отображение

$$
\operatorname{HUR}_{|G|, \mathbf{1}}^{m, G}\left(\mathbb{P}^{1}\right) \rightarrow \operatorname{HUR}_{|G|, \mathbf{1}}^{G}\left(\mathbb{P}^{1}\right)=\operatorname{HUR}^{G}\left(\mathbb{P}^{1}\right)
$$

является сюръективным неразветвленным морфизмом. 
Tеорема 3.6. Неприводимые компоненты пространства $\mathrm{HUR}^{G}\left(\mathbb{P}^{1}\right)$ находятся во взаимно однозначном соответствии с орбитами элементов $s \in$ $S_{G}^{G} \subset S(G, G)$ при действии группь $\operatorname{Aut}(G)$ на полугруппе $S(G, G)$. Ecли $\operatorname{Aut}(G)=G$, то на множестве неприводимых компонент пространства $\operatorname{HUR}^{G}\left(\mathbb{P}^{1}\right)$ имеется естественная структура полугруппы $S_{G, \mathbf{1}}^{G}$.

ДокАЗАтЕльство. Первая часть теоремы следует из следствия 2.5.

Чтобы доказать вторую часть, заметим, что равенство $\operatorname{Aut}(G)=G$ означает, что любой автоморфизм группы $G$ является внутренним. Согласно предложению 1.1 элементы полугруппы $S_{G, 1}^{G}$ остаются неподвижными при действии группы $G$ одновременными сопряжениями. Поэтому согласно следствию 2.5 естественное отображение (3.1) является изоморфизмом, который дает искомую структуру полугруппы на пространстве $\mathrm{HUR}^{G}\left(\mathbb{P}^{1}\right)$.

В частности, теорема 3.6 и следствие 2.4 влекут следующую теорему.

Tеорема 3.7. Неприводимые компоненты пространства $\operatorname{HUR}^{\mathcal{S}_{3}}\left(\mathbb{P}^{1}\right)$ накрытий Галуа с группой Галуа $G=\mathcal{S}_{3}$ однозначно определяются типом разложения монодромии на множители принадлежащих им накрытий.

\section{Список литературы}

1. A. Clebsch, "Zur Theorie der Riemann'schen Fläche", Math. Ann., 6:2 (1873), $216-230$.

2. A. Hurwitz, "Ueber Riemann'sche Flachen mit gegebenen Verzweigungspunkten", Math. Ann., 39:1 (1891), 1-60.

3. W. Fulton, "Hurwitz schemes and irreducibility of moduli of algebraic curves", Ann. of Math. (2), 90:3 (1969), 542-575.

4. M. Fried, R. Biggers, "Moduli spaces of covers and the Hurwitz monodromy group", J. Reine Angew Math., 335 (1982), 87-121.

5. M. D. Fried, H. Völklein, "The inverse Galois problem and rational points on moduli spaces", Math. Ann., 290:1 (1991), 771-800.

6. V. Kanev, "Hurwitz spaces of Galois coverings of $\mathbb{P}^{1}$, whose Galois groups are Weyl groups", J. Algebra, 305:1 (2006), 442-456.

7. P. Kluitmann, "Hurwitz action and finite quotients of braid groups", Braids (Santa Cruz, CA, 1986), Contemp. Math., 78, Amer. Math. Soc., Providence, RI, 1988, 299-325.

8. S. Mochizuki, "The geometry of the compactification of the Hurwitz scheme", Publ. Res. Inst. Math. Sci., 31:3 (1995), 355-441.

9. B. Wajnryb, "Orbits of Hurwitz action for coverings of a sphere with two special fibers", Indag. Math. (N.S.), 7:4 (1996), 549-558.

10. B. Moishezon, M. Teicher, "Braid group technique in complex geometry. I. Line arrangements in $\mathbb{C P}^{2}$ ", Braids (Santa Cruz, CA, 1986), Contemp. Math., 78, Amer. Math. Soc., Providence, RI, 1988, 425-555.

11. Вик. С. Куликов, В. М. Харламов, "О брэйд-монодромных разложениях на множители", Изв. РАН. Сер. матем., 67:3 (2003), 79-118; англ. пер.: V. M. Kharlamov, V. S. Kulikov, "On braid monodromy factorizations", Izv. Math., 67:3 (2003), 499-534. 
12. D. Auroux, "A stable classification of Lefschetz fibrations", Geom. Topol., 9 (2005), 203-217.

13. Вик. С. Куликов, "Кривые Гурвица", УМН, 62:6 (2007), 3-86; англ. пер.: V.S. Kulikov, "Hurwitz curves", Russian Math. Surveys, 62:6 (2007), 1043-1119.

14. Ю. А. Кузьмин, "Об одном способе построения $C$-групп", Изв. РАН. Сер. матем., 59:4 (1995), 105-124; англ. пер.: Yu. V. Kuz'min, "On a method of constructing C-groups", Izv. Math., 59:4 (1995), 765-783.

Вик. С. Куликов (Vik. S. Kulikov)

Математический институт им. В. А. Стеклова РАН

E-mail: kulikov@mi.ras.ru
Поступило в редакцию 15.03.2010 07.07 .2010 